University of Tennessee Health Science Center

UTHSC Digital Commons

\title{
Development of Co-processed Plasticized Cellulose Acetate for Sustained Release Matrix Tablets
}

Yinqi Zhou

University of Tennessee Health Science Center

Follow this and additional works at: https://dc.uthsc.edu/dissertations

Part of the Medicinal and Pharmaceutical Chemistry Commons, and the Pharmaceutics and Drug Design Commons

\section{Recommended Citation}

Zhou, Yinqi , "Development of Co-processed Plasticized Cellulose Acetate for Sustained Release Matrix Tablets" (2012). Theses and Dissertations (ETD). Paper 326. http://dx.doi.org/10.21007/ etd.cghs.2012.0382.

This Dissertation is brought to you for free and open access by the College of Graduate Health Sciences at UTHSC Digital Commons. It has been accepted for inclusion in Theses and Dissertations (ETD) by an authorized administrator of UTHSC Digital Commons. For more information, please contact jwelch30@uthsc.edu. 


\title{
Development of Co-processed Plasticized Cellulose Acetate for Sustained Release Matrix Tablets
}

\author{
Abstract \\ Cellulose Acetate (CA) is a polymer extensively used in pharmaceutical applications. Because of the \\ hydrophobic nature and good film properties of $\mathrm{CA}$, it is a good polymer candidate for sustained release \\ matrix tablets. Sustained release matrix tablets of cellulose acetate can be prepared by direct \\ compression or wet granulation methods. However, previous studies showed that a large amount of CA \\ was required to achieve the desired sustained release profile for a sparingly soluble drug and it was \\ difficult to formulate a highly water soluble drug by using CA as the retarding agent. Some studies \\ concluded that CA is very sensitive to the solubility of the drug and it is not suitable for retarding the \\ release for highly water soluble drugs.
}

There are two aims in our study. One is to modify the physical characteristic of the CA by using a coprocessing technique and increase the capacity of cellulose acetate to control the drug release rate. The other is to modulate the drug release rate from the hydrophobic sustained release tablets and apply these formulation strategies to reduce the burst release and enhance the final release.

Three different process methods including heat treatment, wet granulation and spray drying were used to prepare the co-processed excipients with CA, plasticizer and inert excipient. The physical, flow and compaction properties of the co-processed excipients were evaluated. All the co-processed excipients prepared in this study all showed good flow characteristic. The spray dried process produced high porous particles with large surface area. These particles required the least energy for plastic deformation during compression and formed the tablets with highest mechanical strength. The wet granulated excipients showed moderate plastic deformation capacity and resulting tablets with acceptable tensile strength. The heat treated excipients required more energy to deform under compression pressure than the other two methods and the resulting tablets have poor mechanical properties. For all preparation methods, addition of $10 \%$ plasticizer increases the plasticity of the final excipient and decreases the tablet tensile strength. The tablets containing hydrophilic plasticizer showed better tensile strength than the hydrophobic plasticizer. The tensile strength of the tablets increased at low plasticizer concentration and the reverse effect was observed on higher plasticizer concentration. Both wet granulated and spray dried excipients showed desired flowability and acceptable compactability which makes them good excipient candidates for direct compression formulation.

The sustained release characteristic of the co-processed excipients were evaluated by a freely water soluble drug propranolol hydrochloride. The spray dried co-processed excipients demonstrated slower drug release profile than the excipients prepared by the other two processing methods. The surface morphology and initial water penetration study found the spray dried co-processed excipient can form a continuous film like structure on the tablet surface which can effectively prevent water penetration and drug diffusion. The drug release rate from the matrix tablets containing the novel spray dried excipients can be decreased by increasing the hydrophobicity of the plasticizer and plasticizer concentration. The drug release rate from the matrix was affected by the compression force but not the $\mathrm{pH}$ of the dissolution medium. Based on porosity results and drug release mechanism study, addition of plasticizer to the coprocessed excipients showed a decrease in the drug release rate due to three reasons: a) decrease in the porosity of the tablet; b) decrease in the effective diffusion coefficient; $c$ ) assist in maintaining tablet structure during dissolution.

Three different types of pore formers were tried to modulate drug release characteristics from the plastic inert matrix. With the help of pore formers lactose and Starch1500®, both observed improved final release as well as increased burst release. When a small amount of hydrophilic polymer pore former was 
incorporated into the formulation, it showed a limited burst release initially and complete release at final stage. The optical microscopy results revealed the initial gel formation on the tablet surface with development of a swollen porous structure in the matrix network during dissolution. The drug release rate from this swellable porous matrix system is independent with the type and the particle size of hydrophilic polymer and the $\mathrm{pH}$ of dissolution medium. The drug release rate increased when the hydrophilic polymer concentration was less than $5 \%$. Further increase of the hydrophilic polymer concentration to $10 \%$ did not further increase the drug release rate. The low viscosity grade hydrophilic polymer resulted in rapid burst release and incomplete final release, while middle to high viscosity grades of hydrophilic polymer avoided these problems. The drug release is controlled by both drug diffusion and polymer relaxation. The Fickian diffusion is the dominant release mechanism at the initial stage and the polymer relaxation becomes dominant thereafter.

The spray dried co-processed excipient and the swollen porous matrix system were used to develop the sustained release matrix formulation for three different drugs with different solubility. The optimized formulations for these three drugs either meet the USP specifications or have similar dissolution profile to the commercial product. For a freely water soluble drug propranolol hydrochloride, a roller compaction method was applied to reduce the initial burst release. The tablets prepared by the roller compacted granules showed suppressed burst release and complete final release. For the sparingly water soluble drug theophylline, incorporating $10 \%$ hydrophilic polymer can successfully reduce the burst release as well as improve the final release. Two successful sustained release formulations of the poorly water soluble drug glipizide were obtained by using the plasticized co-processed excipient. Both of them have similar dissolution profile as the commercial product Gluctrol XL®. But the formulation prepared with lactose as pore former showed a large variation in dissolution profile while another formulation prepared with both lactose and hydrophilic polymer as pore formers exhibited less variation in dissolution profile and a higher $\mathrm{f} 2$ value.

In conclusion, the spray dried co-processed plasticized cellulose acetate developed in this study exhibits good physical and mechanical properties. It can be used alone or combined with hydrophilic polymers to control the release rate of the drugs with different solubility.

\section{Document Type}

Dissertation

Degree Name

Doctor of Philosophy (PhD)

\section{Program}

Pharmaceutical Sciences

\section{Research Advisor}

James R. Johnson, Ph.D.

\section{Keywords}

Cellulose acetate, co-processed excipient, matrix tablet, sustained release

\section{Subject Categories}

Medicinal and Pharmaceutical Chemistry | Medicine and Health Sciences | Pharmaceutics and Drug Design | Pharmacy and Pharmaceutical Sciences 
Comments

One year embargo expired May 2013

This dissertation is available at UTHSC Digital Commons: https://dc.uthsc.edu/dissertations/326 


\title{
Development of Co-processed Plasticized Cellulose Acetate for Sustained Release
} Matrix Tablets

\author{
A Dissertation \\ Presented for \\ The Graduate Studies Council \\ The University of Tennessee \\ Health Science Center
}

In Partial Fulfillment

Of the Requirements for the Degree

Doctor of Philosophy

From The University of Tennessee

By

Yinqi Zhou

December 2012 
Copyright (C) 2012 by Yinqi Zhou. All rights reserved. 


\section{DEDICATION}

I dedicate this work to my parents

Mrs. Ruohui Wang and Mr. Shuxuan Zhou and my husband Li Ou my daughter Iris $\mathrm{Ou}$

For their love and support 


\section{ACKNOWLEDGEMENTS}

When I sit down and try to write down the names of those people to whom I owe my gratitude, I find out that the list is such a long one!

First, my sincerest gratitude goes to my beloved professor Dr. Atul J. Shukla, for his guidance, encouragement, motivation and support given to me during the course of this research work. I will always value his advice, honesty and friendship, and it has truly been a pleasure working with him.

I would extend my special thanks and acknowledgement to Dr. James R. Johnson who agreed to mentor me and offer his guidance and support after the sad demise of Dr. Shukla. He led me through the completion of this research project work. My Ph.D work won't be finished without his guidance and support.

I would also like to thank the members of my research committee: Dr. George C. Wood, Dr. William McLaughlin and Dr. Hassan Almoazen for their suggestions, advice and help.

I have been very lucky that I have met many people who give me their support during the past five years, Dr. Chaoju Xiao, Dr. Maria Gerald Rajan, Dr. Paras Jain, Dr. Namrata, Dr. Sonia, Dr. Weiqiang Cheng, Dr. Wen Qu, Dr. Wenli Lu, Dr. Xiao Zhao, Jin $\mathrm{Xu}$, Frank Zhang, Rui Zhu and Suresh Potharaju, I am really grateful to the Shukla/Johnson group and they have made my study and research at UT an enjoyable one.

I would like to thank the University of Tennessee Health Science Center for providing the opportunity and financial assistance to pursue a Doctor of Philosophy degree, and the entire faculty and staff in Department of Pharmaceutical Sciences for their help and support during my graduate study.

Nothing in words could have expressed my deepest appreciation to my family. My parents, Mr. Shuxuan Zhou and Mrs. Ruohui Wang; my husband, Li Ou and my lovely daughter, Iris Ou. I am here because of them. 


\begin{abstract}
Cellulose Acetate (CA) is a polymer extensively used in pharmaceutical applications. Because of the hydrophobic nature and good film properties of CA, it is a good polymer candidate for sustained release matrix tablets. Sustained release matrix tablets of cellulose acetate can be prepared by direct compression or wet granulation methods. However, previous studies showed that a large amount of CA was required to achieve the desired sustained release profile for a sparingly soluble drug and it was difficult to formulate a highly water soluble drug by using CA as the retarding agent. Some studies concluded that $\mathrm{CA}$ is very sensitive to the solubility of the drug and it is not suitable for retarding the release for highly water soluble drugs.

There are two aims in our study. One is to modify the physical characteristic of the CA by using a co-processing technique and increase the capacity of cellulose acetate to control the drug release rate. The other is to modulate the drug release rate from the hydrophobic sustained release tablets and apply these formulation strategies to reduce the burst release and enhance the final release.

Three different process methods including heat treatment, wet granulation and spray drying were used to prepare the co-processed excipients with CA, plasticizer and inert excipient. The physical, flow and compaction properties of the co-processed excipients were evaluated. All the co-processed excipients prepared in this study all showed good flow characteristic. The spray dried process produced high porous particles with large surface area. These particles required the least energy for plastic deformation during compression and formed the tablets with highest mechanical strength. The wet granulated excipients showed moderate plastic deformation capacity and resulting tablets with acceptable tensile strength. The heat treated excipients required more energy to deform under compression pressure than the other two methods and the resulting tablets have poor mechanical properties. For all preparation methods, addition of $10 \%$ plasticizer increases the plasticity of the final excipient and decreases the tablet tensile strength. The tablets containing hydrophilic plasticizer showed better tensile strength than the hydrophobic plasticizer. The tensile strength of the tablets increased at low plasticizer concentration and the reverse effect was observed on higher plasticizer concentration. Both wet granulated and spray dried excipients showed desired flowability and acceptable compactability which makes them good excipient candidates for direct compression formulation.
\end{abstract}

The sustained release characteristic of the co-processed excipients were evaluated by a freely water soluble drug propranolol hydrochloride. The spray dried co-processed excipients demonstrated slower drug release profile than the excipients prepared by the other two processing methods. The surface morphology and initial water penetration study found the spray dried co-processed excipient can form a continuous film like structure on the tablet surface which can effectively prevent water penetration and drug diffusion. The drug release rate from the matrix tablets containing the novel spray dried excipients can be decreased by increasing the hydrophobicity of the plasticizer and 
plasticizer concentration. The drug release rate from the matrix was affected by the compression force but not the $\mathrm{pH}$ of the dissolution medium. Based on porosity results and drug release mechanism study, addition of plasticizer to the co-processed excipients showed a decrease in the drug release rate due to three reasons: a) decrease in the porosity of the tablet; b) decrease in the effective diffusion coefficient; c) assist in maintaining tablet structure during dissolution.

Three different types of pore formers were tried to modulate drug release characteristics from the plastic inert matrix. With the help of pore formers lactose and Starch1500®, both observed improved final release as well as increased burst release. When a small amount of hydrophilic polymer pore former was incorporated into the formulation, it showed a limited burst release initially and complete release at final stage. The optical microscopy results revealed the initial gel formation on the tablet surface with development of a swollen porous structure in the matrix network during dissolution. The drug release rate from this swellable porous matrix system is independent with the type and the particle size of hydrophilic polymer and the $\mathrm{pH}$ of dissolution medium. The drug release rate increased when the hydrophilic polymer concentration was less than $5 \%$. Further increase of the hydrophilic polymer concentration to $10 \%$ did not further increase the drug release rate. The low viscosity grade hydrophilic polymer resulted in rapid burst release and incomplete final release, while middle to high viscosity grades of hydrophilic polymer avoided these problems. The drug release is controlled by both drug diffusion and polymer relaxation. The Fickian diffusion is the dominant release mechanism at the initial stage and the polymer relaxation becomes dominant thereafter.

The spray dried co-processed excipient and the swollen porous matrix system were used to develop the sustained release matrix formulation for three different drugs with different solubility. The optimized formulations for these three drugs either meet the USP specifications or have similar dissolution profile to the commercial product. For a freely water soluble drug propranolol hydrochloride, a roller compaction method was applied to reduce the initial burst release. The tablets prepared by the roller compacted granules showed suppressed burst release and complete final release. For the sparingly water soluble drug theophylline, incorporating $10 \%$ hydrophilic polymer can successfully reduce the burst release as well as improve the final release. Two successful sustained release formulations of the poorly water soluble drug glipizide were obtained by using the plasticized co-processed excipient. Both of them have similar dissolution profile as the commercial product Gluctrol XL $\AA$. But the formulation prepared with lactose as pore former showed a large variation in dissolution profile while another formulation prepared with both lactose and hydrophilic polymer as pore formers exhibited less variation in dissolution profile and a higher $f_{2}$ value.

In conclusion, the spray dried co-processed plasticized cellulose acetate developed in this study exhibits good physical and mechanical properties. It can be used alone or combined with hydrophilic polymers to control the release rate of the drugs with different solubility. 


\section{TABLE OF CONTENTS}

Chapter 1. Introduction........................................................................................................1

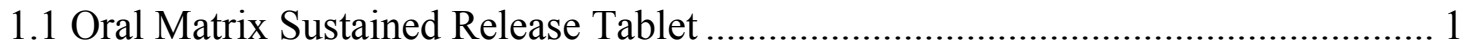

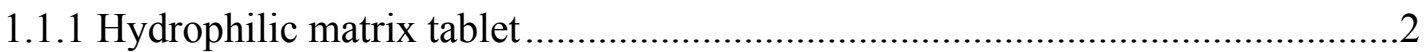

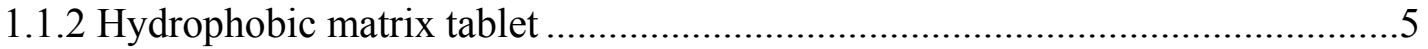

1.2 Mathematic Models for Matrix System ……………............................................. 7

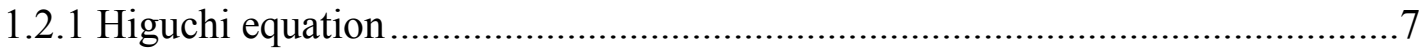

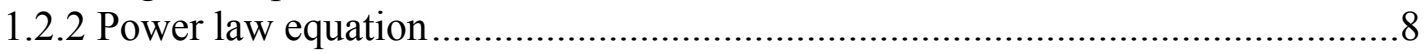

1.2.3 Hopfenberg model .................................................................................

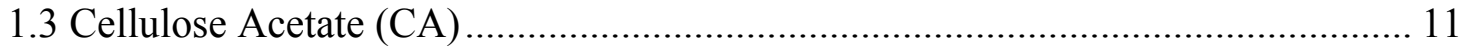

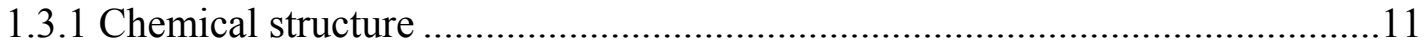

1.3.2 Physicochemical properties of CA...............................................................

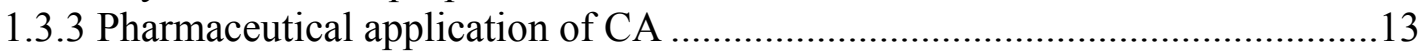

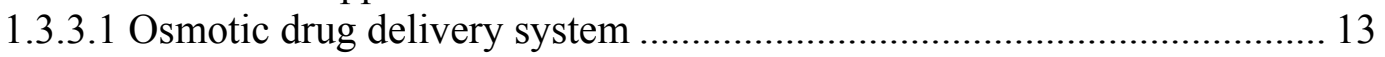

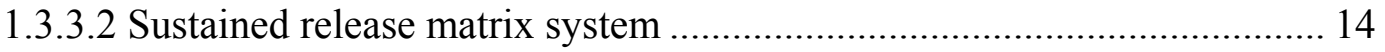

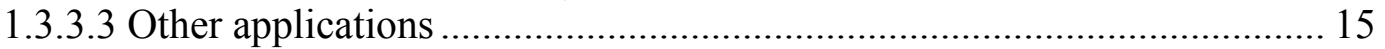

1.4 Application of Plasticizers on Oral Solid Dosage Form........................................ 15

1.5 Co-processed Excipients for Oral Solid Dosage Forms ……………....................... 17

1.5.1 Excipient for oral solid dosage form.......................................................... 17

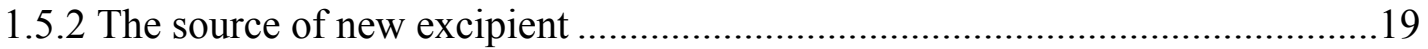

1.5.3 Co-processed excipient ........................................................................19

1.5.4 New excipients in sustained release application ............................................20

Chapter 2. Preparation of Co-processed Plasticized Cellulose Acetate Excipient and Evaluation of the Physical and Compaction Properties ............................................23

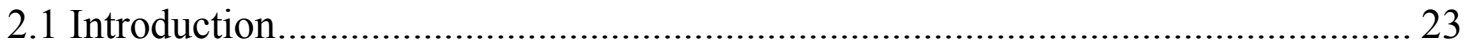

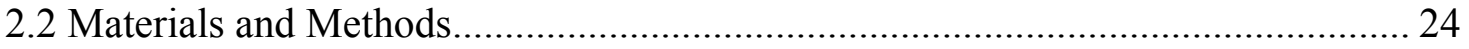

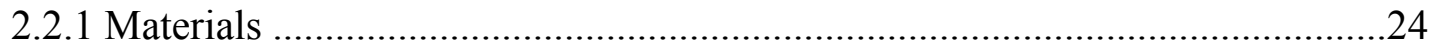

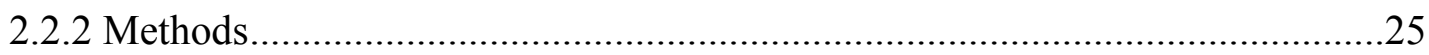

2.2.2.1 Co-processed methods .......................................................................... 25

2.2.2.2 Bulk density, tap density and Carr's index evaluation .............................. 27

2.2.2.3 Powder porosity evaluation................................................................. 27

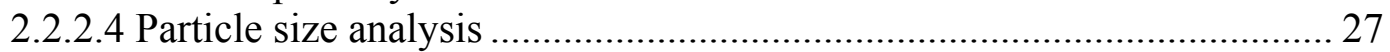

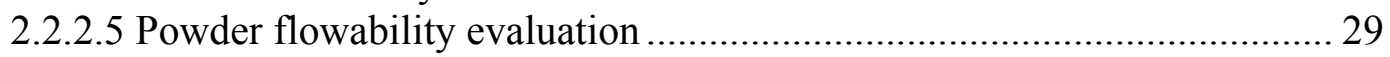

2.2.2.6 Specific surface area measurement ........................................................... 29

2.2.2.7 Scanning electron microscopy (SEM) study ............................................. 29

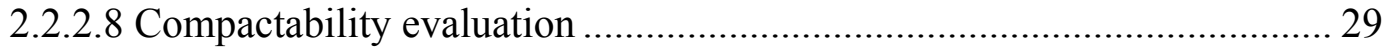

2.2.2.9 Glass transition temperature $(\mathrm{Tg})$ measurement ....................................... 30

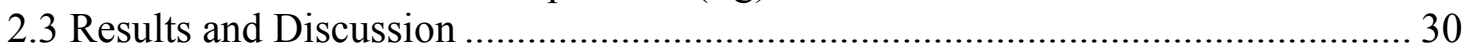

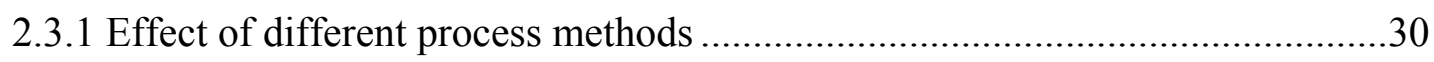

2.3.1.1 Morphology ......................................................................................... 30

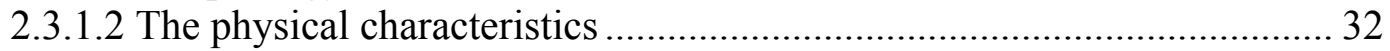

2.3.1.3 Flow characteristics .......................................................................... 34

2.3.1.4 Compactability …………………........................................................ 34 
2.3.1.4.1 Compression profile ......................................................................... 34

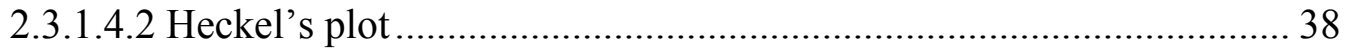

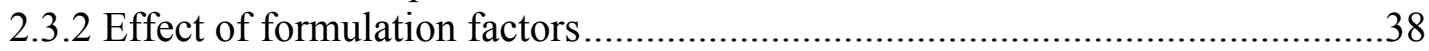

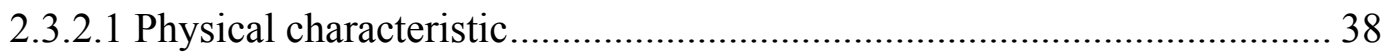

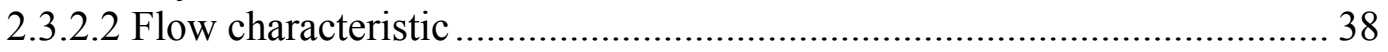

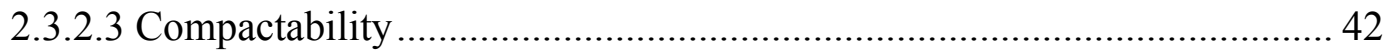

2.3.2.3.1 Compression profile.................................................................... 42

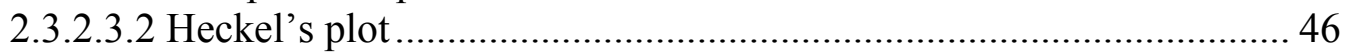

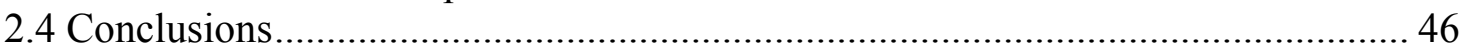

\section{Chapter 3. Sustained Release Properties and Drug Release Mechanism Study of} a Novel Co-processed Excipient...............................................................................................49

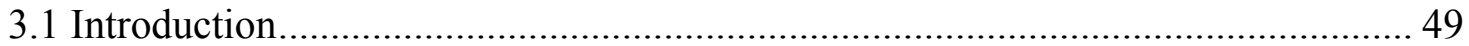

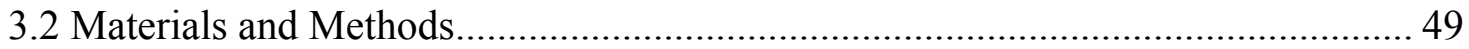

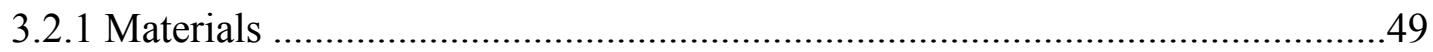

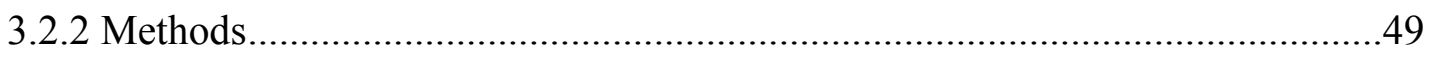

3.2.2.1 Tablets preparation................................................................................... 49

3.2.2.2 In vitro dissolution study .................................................................... 51

3.2.2.3 Tablet porosity measurement ............................................................ 51

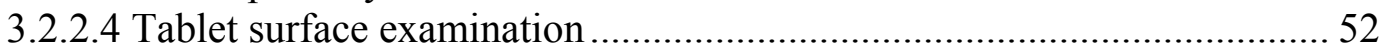

3.2.2.5 Initial water penetration study ............................................................ 52

3.2.2.6 Drug solubility measurement ............................................................ 52

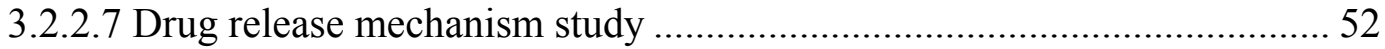

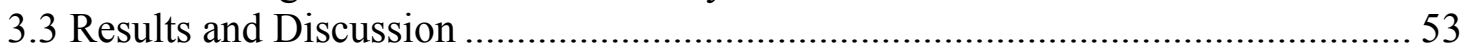

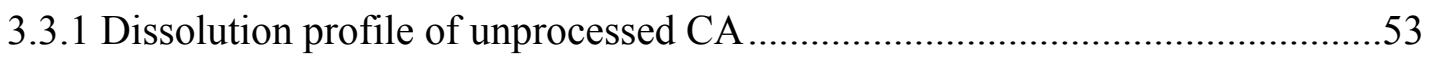

3.3.2 The effect of preparation process of co-processed excipients on drug

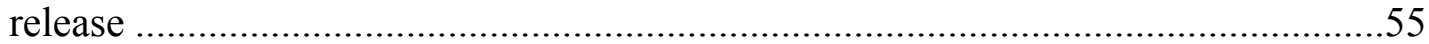

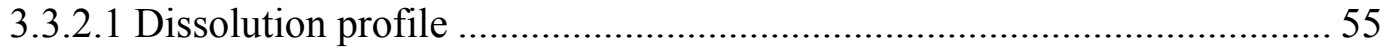

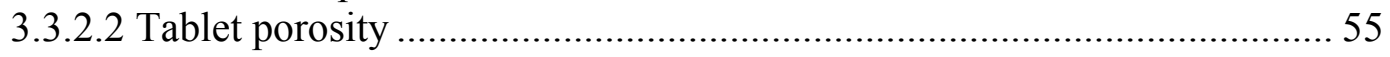

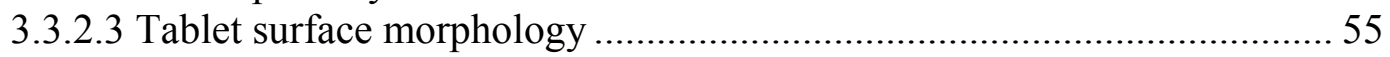

3.3.2.4 Initial water penetration study ................................................................ 59

3.3.3 The effect of co-processed excipients' formulation on drug release ................59

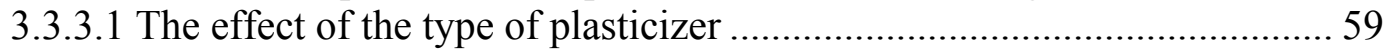

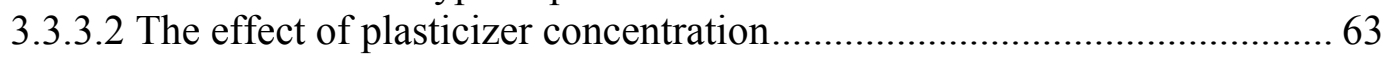

3.3.4 The effect of compression force on drug dissolution.........................................63

$3.3 .5 \mathrm{pH}$ effect of dissolution medium on drug dissolution profile ............................63

3.3.6 Drug solubility effect on drug dissolution profile.............................................63

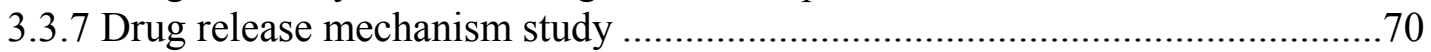

3.3.7.1 Higuchi model.................................................................................... 70

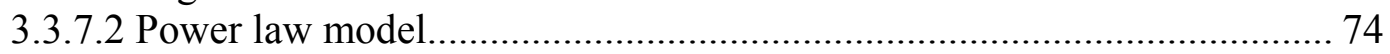

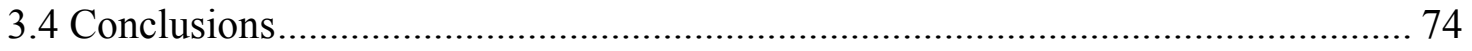

\section{Chapter 4. Hydrophilic Polymer Effect on the Plasticized Cellulose Acetate}

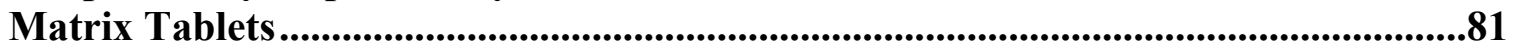

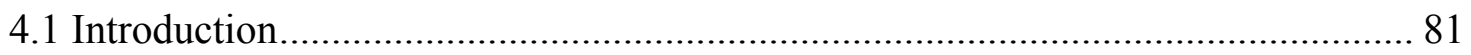

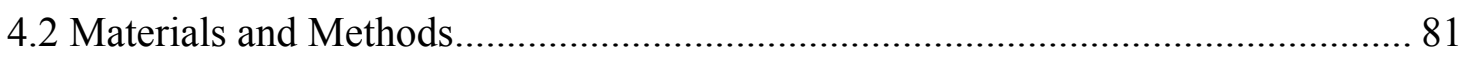




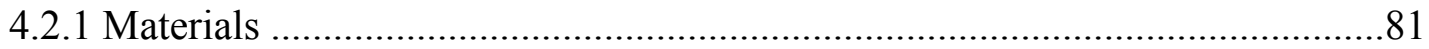

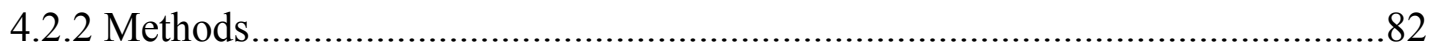

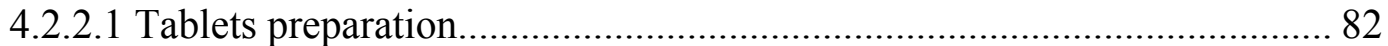

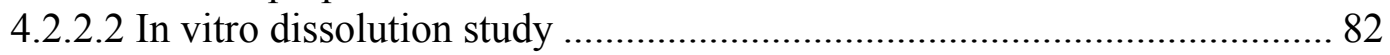

4.2.2.3 Optical microscopic examination .............................................................. 86

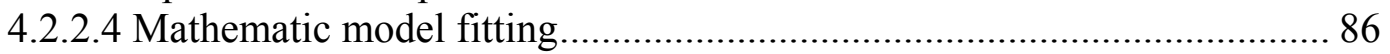

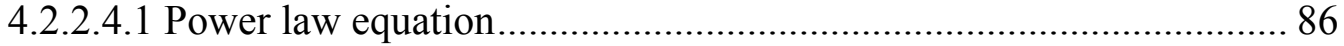

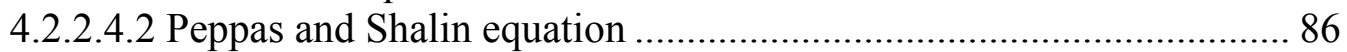

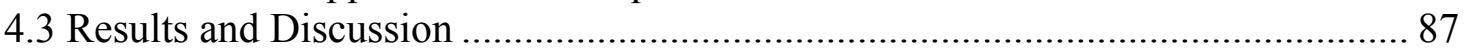

4.3.1 The effect of different pore formers on drug dissolution...................................87

4.3.2 The effect of the concentration of hydrophilic polymer on drug dissolution ...89

4.3.3 The type of hydrophilic polymer effect on drug dissolution ............................89

4.3.4 The effect of hydrophilic polymer viscosity grades on drug dissolution..........92

4.3.5 The particle size of hydrophilic polymer effect on drug dissolution .................92

4.3.6 The effect of formulation factors of co-processed excipient on drug

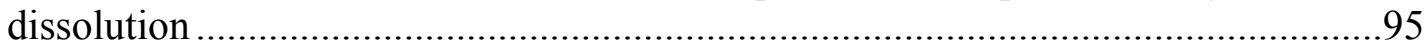

4.3.7 The effect of $\mathrm{pH}$ of dissolution medium on drug dissolution ...........................95

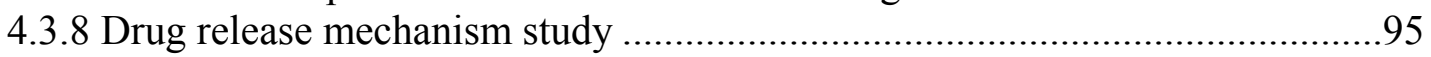

4.3.8.1 Optical microscopic observation.......................................................... 95

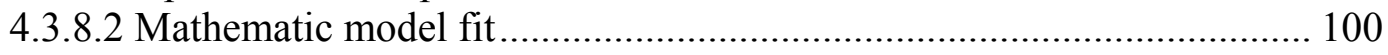

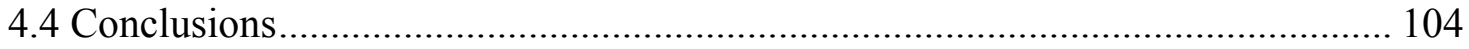

\section{Chapter 5. Application of the Novel Matrix System on the Drugs with Different} Solubility ................................................................................................................................105

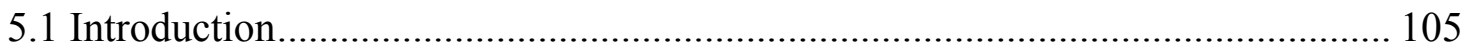

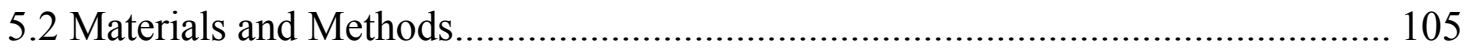

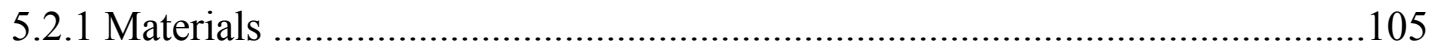

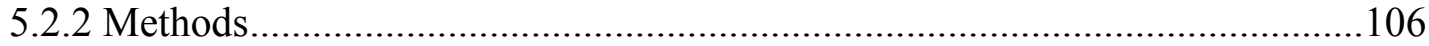

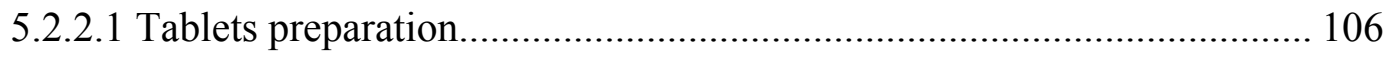

5.2.2.1.1 Propranolol hydrochloride tablets................................................... 106

5.2.2.1.2 Theophylline anhydrous tablets ...................................................... 106

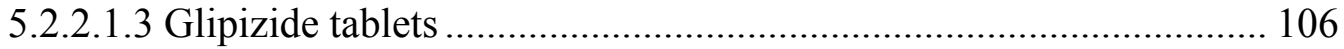

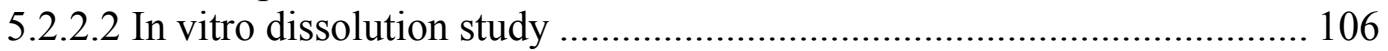

5.2.2.2.1 Propranolol hydrochloride and theophylline .................................. 106

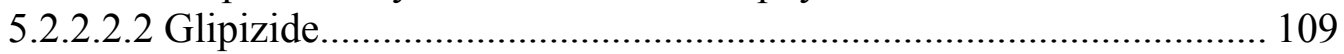

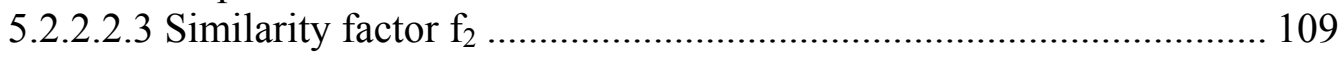

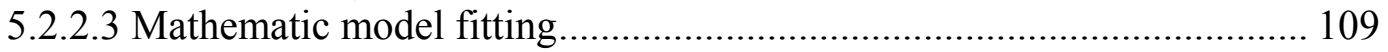

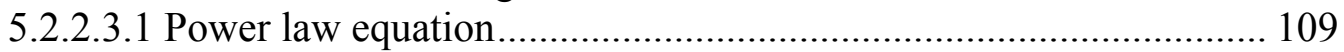

5.2.2.3.2 Peppas and Shalin equation .......................................................... 110

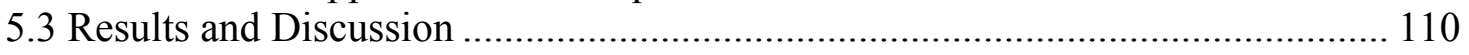

5.3.1 Propranolol hydrochloride matrix tablets .......................................................110

5.3.1.1 The effect of roller compaction process on drug release ......................... 111

5.3.1.2 The effect of the intragranular polymer on drug release........................... 111

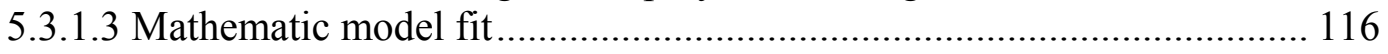

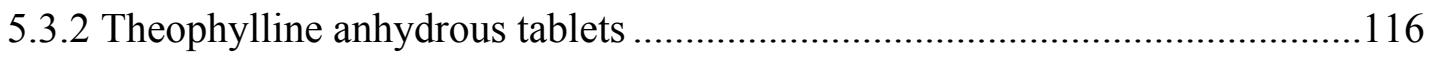

5.3.2.1 Formulation development ................................................................ 116 
5.3.2.2 Mathematic model fit

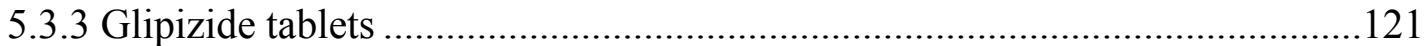

5.3.3.1 The effect of co-processed excipient on drug release ........................... 121

5.3.3.2 The effect of hydrophilic polymer on drug release.............................. 125

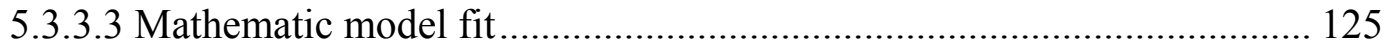

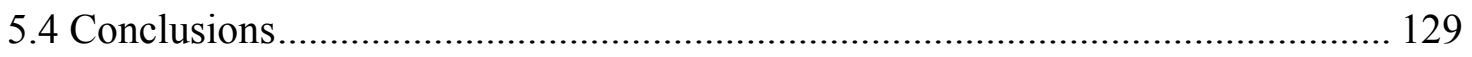

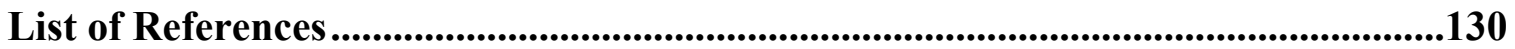

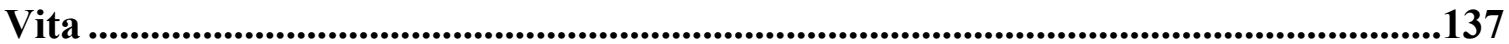




\section{LIST OF TABLES}

Table 1-1. Release exponent " $\mathrm{n}$ " for devices with different geometry ............................9

Table 1-2. Physical and chemical properties of different grade of cellulose acetate .....12

Table 1-3. Physical and chemical properties of commonly used pharmaceutical

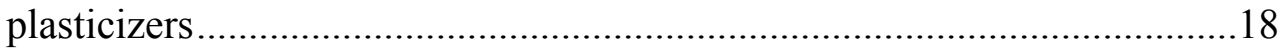

Table 1-4. Commercial available co-processed excipients ...............................................

Table 2-1. Formulations of the co-processed excipients for three preparation

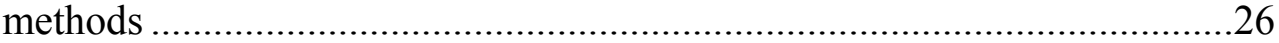

Table 2-2. Formulations of the co-processed excipients prepared from different plasticizers ............................................................................................28

Table 2-3. Formulations of the co-processed excipients prepared from different concentration of plasticizers......................................................................28

Table 2-4. Physical properties for the co-processed excipient prepared by three different methods

Table 2-5. Flow properties for the co-processed excipient prepared by three different methods

Table 2-6. Specific surface area of the co-processed excipients prepared by three different methods

Table 2-7. Physical properties for the co-processed excipients prepared from different plasticizers by using spray dried method

Table 2-8. Physical properties for the co-processed excipients prepared from different concentrations of plasticizer by using spray dried method

Table 2-9. Flow properties for the co-processed excipient prepared by different plasticizers by using spray dried method

Table 2-10. Flow properties for the co-processed excipient prepared by different concentrations of plasticizer by using spray dried method

Table 3-1. Formulations of the tablets prepared from the unprocessed CA....................50

Table 3-2. Porosity of the tablets prepared by propranolol hydrochloride and different co-processed excipients

Table 3-3. Porosity of tablets prepared by propranolol hydrochloride and coprocessed excipients with different plasticizers 
Table 3-4. Porosity of tablets prepared by propranolol and co-processed excipients with different concentration of ATBC

Table 3-5. Porosity of tablets prepared by propranolol and spray dried excipient F2 at different compression force....

Table 3-6. Solubility of different drugs in dissolution medium at $37^{\circ} \mathrm{C}$

Table 3-7. Higuchi release constant and effective diffusion coefficient for different drugs in different matrices

Table 3-8. Tablet size change after dissolution..........................................................

Table 4-1. Formulations for tablets prepared with different type of pore formers .........83

Table 4-2. Formulations for tablets prepared with different concentrations of hydrophilic polymer....

Table 4-3. Formulations for tablets prepared with different hydrophilic polymers ........84

Table 4-4. Formulations for tablets prepared with different viscosity grades of hydrophilic polymer

Table 4-5. Formulations for tablets prepared with different co-processed excipients and hydrophilic polymer Polyox 303

Table 4-6. Formulations for tablets prepared with different co-processed excipients and hydrophilic polymer Polyox 303

Table 4-7. Tablets size change during dissolution 101

Table 5-1. Formulations for propranolol hydrochloride tablets prepared with different intragranular hydrophilic polymers

Table 5-2. Formulations for propranolol hydrochloride tablets prepared with intragranular and extragranular hydrophilic polymers

Table 5-3. Formulations of theophylline tablets prepared with different concentrations of hydrophilic polymers 108

Table 5-4. Formulations of glipizide tablets with different concentrations of coprocessed excipients

Table 5-5. Formulations of glipizide tablets prepared with different concentrations of sodium alginate 108

Table 5-6. The drug release results of formulations containing intragranular hydrophilic polymers at 1 hour and 12 hours 
Table 5-7. The drug release results of formulations containing intragranular and extragranular hydrophilic polymers at 1 hour and 12 hours ......................115

Table 5-8. Comparison of propranolol hydrochloride tablets prepared from PR1 and USP specifications ………………………………………………….......115

Table 5-9. Comparison of theophylline tablets prepared from T3 and USP specifications.... 


\section{LIST OF FIGURES}

Figure 1-1. Chemical structure of cellulose acetate.....................................................12

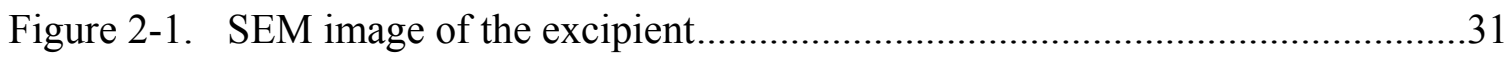

Figure 2-2. Compression profile of the co-processed excipients prepared by three

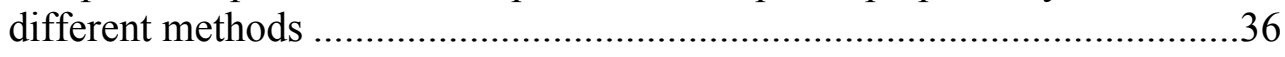

Figure 2-3. Heckel's plot of the co-processed excipients prepared by three different methods

Figure 2-4. Compression profile of the co-processed excipients prepared from different plasticizers by using spray dried method

Figure 2-5. Compression profile of the co-processed excipients prepared from different concentrations of plasticizer by using spray dried method

Figure 2-6. Glass transition temperature of CA with different plasticizers.

Figure 2-7. Heckel's plot of the co-processed excipients prepared from different plasticizers by using spray dried method

Figure 2-8. Heckel's plot of the co-processed excipients prepared from different concentrations of plasticizer by using spray dried method

Figure 3-1. Dissolution profile of the tablets containing unprocessed CA

Figure 3-2. Dissolution profile of tablets containing the co-processed excipients prepared from different process methods .56

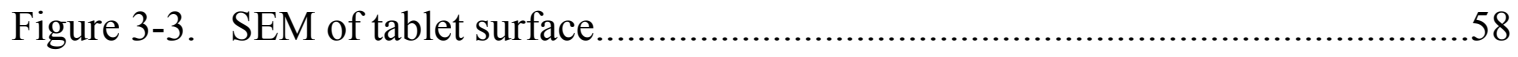

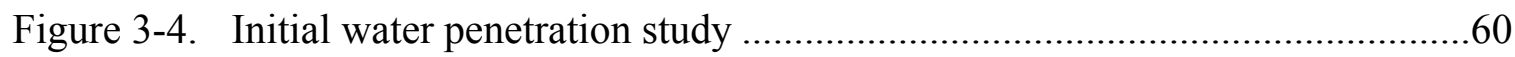

Figure 3-5. The effect of the type of plasticizer of co-processed excipient on the drug release

Figure 3-6. The effect of plasticizer concentration of co-processed excipient on the drug release

Figure 3-7. Compression force effect on drug release..................................................66

Figure 3-8. pH effect on drug release profile ………………………………………....68

Figure 3-9. Drug solubility effect on dissolution profile ................................................71 
Figure 3-10. Higuchi model fitting for tablets prepared from co-processed excipient F0

Figure 3-11. Higuchi model fitting for tablets prepared from the co-processed excipient F2.

Figure 3-12. Power law model fitting for tablets prepared from the co-processed excipient F0.

Figure 3-13. Power law model fitting for tablets prepared from the co-processed excipient F2....

Figure 3-14. Pictures of tablet containing 20\% propranolol hydrochloride and spray dried excipient F0..

Figure 4-1. The effect of the pore formers on drug dissolution.

Figure 4-2. The effect of the concentration of hydrophilic polymer on drug dissolution .90

Figure 4-3. The effect of different hydrophilic polymers on drug dissolution .91

Figure 4-4. The effect of different viscosity of hydrophilic polymer on drug dissolution .....

Figure 4-5. The effect of particle size of hydrophilic polymer on drug dissolution........94

Figure 4-6. The effect of co-processed excipients on drug dissolution........................96

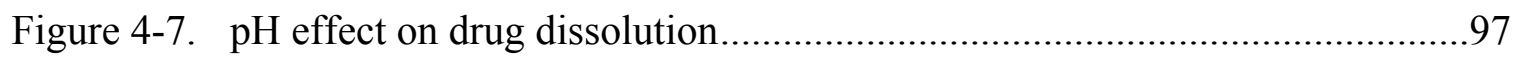

Figure 4-8. Microscopic picture of colored tablets (H0) during dissolution ..................98

Figure 4-9. Microscopic picture of colored tablets (H2) during dissolution ..................99

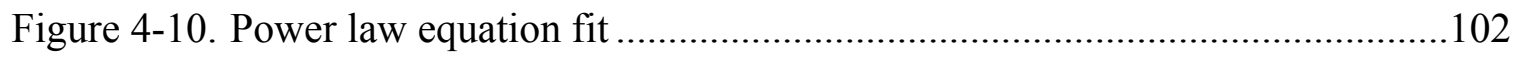

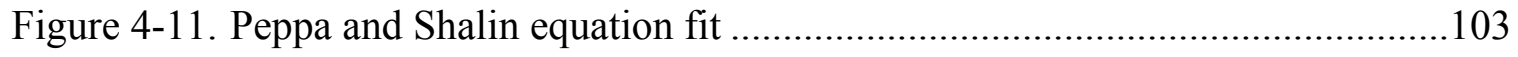

Figure 5-1. Effect of roller compaction on propranolol hydrochloride release.............112

Figure 5-2. Effect of extragranular hydrophilic polymer on propranolol hydrochloride release

Figure 5-3. Power law equation fit of propranolol hydrochloride release profile

Figure 5-4. Peppas and Shalin equation fit of propranolol hydrochloride release profile 
Figure 5-5. Effect of hydrophilic polymer concentrations on theophylline release ......119

Figure 5-6. Power law equation fit of theophylline release profile ..............................122

Figure 5-7. Peppas and Shalin equation fit of theophylline release profile...................123

Figure 5-8. Effect of concentrations of co-processed excipient on glipizide release ....124

Figure 5-9. Effect of hydrophilic polymer concentrations on glipizide release ............126

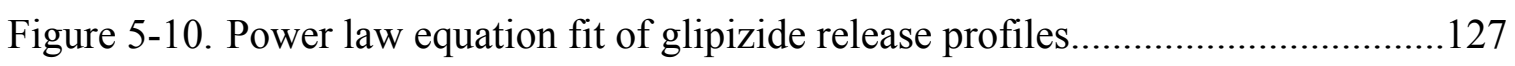

Figure 5-11. Peppas and Shalin equation fit of glipizide release profiles .....................128 


\section{List of Abbreviations}

\begin{tabular}{ll} 
ATBC & Acetyltributyl Citrate \\
ATEC & Acetyltriethyl Citrate \\
CA & Cellulose Acetate \\
EC & Ethyl Cellulose \\
DBS & Dibutyl Sebacate \\
DCP & Di Calcium Phosphate \\
DSC & Differential Scanning Calorimetry \\
HEC & Hydroxyethyl Cellulose \\
HPC & Hydroxypropyl Cellulose \\
HPMC & Hydroxypropyl Methylcellulose \\
Hr & Hour \\
MC & Micro Crystalline Cellulose \\
Min & Minutes \\
mT & Metric Tons \\
PEG & Polyethylene Glycol \\
PEO & Polyethylene Oxide \\
PVP & Polyvinyl Pyrrolidone \\
RPM & Revolutions Per Minute \\
SA & Sodium Alginate \\
Sec & Seconds \\
SEM & Scanning Electron Microscopy \\
SGF & Simulated Gastric Fluid \\
SIF & Simulated Intestinal Fluid \\
TBC & Tributyl Citrate \\
TEC & Triethyl Citrate \\
Tg & Glass Transition Temperature \\
USP & United States Pharmacopeia \\
UV & Ultra Violet \\
\hline
\end{tabular}




\section{Chapter 1. Introduction}

Oral drug delivery is the most preferred route for delivering drugs to the body. The popularity is due to the distinct advantages such as convenient to use, high patient compliance, easy manufacture and relatively low cost. Traditional oral drug delivery systems including oral suspensions, oral solutions, immediate release tablets and capsules often cannot maintain the constant drug concentration in the blood, therefore frequent doses were required for the drugs with a short half-life. In addition side effects may also occur for the drugs with a narrow therapeutic window. Oral sustained release systems have been developed since 1950 for solving these problems. These systems are designed to control the drug release rate from gastrointestinal tract to extend the duration of action and minimize the fluctuation of drug concentration and achieve better therapeutic performance. In addition to the clinical advantages, the sustained release system can also help to extend the product life-cycle. That becomes an important strategy for management of product life-cycle for pharmaceutical industry.

The first sustained release product was developed in 1950 using the trade name Spansules ${ }^{\circledR}[1]$. It was a capsule form which contained a large number of beads. The sustained release profile was achieved by coating a hydrophobic wax on beads containing a sugar core and drug layer. Over the last six decades, a great number of sustained release products were commercialized and a number of new sophisticated technologies were developed and introduced to this area. The major commercialized sustained release systems can be summarized as: matrix system, reservoir system and osmotic system. Drug release is generally controlled by one or a combination of the following mechanisms: drug diffusion, system swelling, system erosion or osmotic pressure.

Among all the sustained release systems, the matrix system is the simplest and the most widely used sustained release system. This is due to the cost effective manufacture process and the accommodation for a wide range of drugs with different chemical and physical properties. The majority of the commercialized matrix systems are in the form of tablets. The preparation process and equipment for preparation of matrix tablets is usually similar to the conventional tablets.

\subsection{Oral Matrix Sustained Release Tablet}

In a matrix tablet, the drug is uniformly blended with other excipients including rate-controlling material(s) and other inactive ingredients. Drug release is controlled either by drug diffusion and/or matrix erosion. Based on the properties of rate-controlling material, the matrix system can be divided into two types: hydrophilic matrix and hydrophobic matrix. 


\subsubsection{Hydrophilic matrix tablet}

In a hydrophilic matrix tablet, the rate-controlling material is the hydrophilic polymer. Upon contact with the aqueous medium or gastrointestinal fluid, the water penetrates into the free spaces between the chains of hydrophilic polymer. The rigid polymer relaxes and become flexible. A glassy to rubbery phase transformation of the hydrophilic polymer occurs. As a result, the tablet swells and a viscous gel layer is formed on the surface of tablet. The tablet consists of two different states: the glassy core and rubbery gel layer. The lengthened and torturous diffusion pathway of the viscous gel layer can hinder the release of embedded drug. As more water enters the tablet, the concentration of the hydrophilic polymer at the surface dilutes and the disentanglement concentration can be reached. The concentration at which polymeric chains are disentangled is related to the rheological properties of the gels and corresponds to the tablet erosion. The tablet can be completely eroded when water continues to penetrate towards the core of the tablet through the gel layer. During dissolution, three driving forces are present in the polymer network: the penetrating concentration gradient, polymer concentration gradient and osmotic force. Drug release from the hydrophilic matrices is controlled by diffusion and/or polymer erosion.

There are three broad categories of polymers that commonly used in the preparation of hydrophilic matrices.

a) Cellulose derivatives: methylcellulose (MC); hydroxyethylcellulose (HEC); hydroxypropylcellulose (HPC); hydroxypropylmethylcellulose (HPMC); and sodium carboxymethylcellulose (NaCMC).

b) Natural polymers: sodium alginate; xanthan gum; carrageenan; locust bean gum; chitosan; guar gum; pectin and modified starches.

c) Synthetic polymers: polyacrylic acid derivative (carbopol); polyethylene oxide (PEO), polyvinyl pyrrolidone (PVP).

These polymers also can be divided into ionic and non-ionic. Non-ionic polymers such as HPMC and PEO will not be ionized in the water and their hydration and erosion are independent of $\mathrm{pH}$. On the contrary, the ionic polymers such as chitosan and Carbopol exhibit $\mathrm{pH}$ dependent hydrophilicity due to ionization of the polymer chain under a certain $\mathrm{pH}$.

Of all the polymers, HPMC is one of the most widely used polymers in hydrophilic matrices. It is cellulose ether containing methoxyl and hydroxypropyl groups. Different substitutions and viscosity grades of HPMC are commercially available. The key features and advantages of HPMC included [2]:

a) Non-ionic polymer resulting in $\mathrm{pH}$ independent drug release performance.

b) Stable within a wide $\mathrm{pH}$ range and resistant to enzymatic degradation.

c) Less chance for chemical interaction or complexation with other formulation components.

d) Accommodation of various drugs and a high level of drug loading. 
e) Good compaction characteristics in both direct compression and granulation.

f) Easy to manufacture.

Another widely used hydrophilic polymer is carbopol. It is a synthetic high molecular weight ionic polymer prepared by crosslinking acrylic acid with allyl sucrose or allylpentaerythritol. Carbopol is also available with different viscosity and particle size grades such as 934P, 974P, 971P and 71G [3]. They are not soluble, but swellable in water. Owing to the present of carboxyl group in the polymer chain, the swellability of carbopol polymers is $\mathrm{pH}$ dependent and the maximum swellability is 1,000 times their original volume at pH6.0 [4]. Unlike the traditional linear polymer like HPMC and HPC which form continuous gel layer by the single entangled polymer chains, the crosslinked carbopol polymer will form a discrete microgel made of many polymer particles. The drug can be entrapped into this microgel and slowly release by diffusion. Since carbopol doesn't dissolve in the water, the erosion doesn't take place in this system. When the system is fully hydrated, the osmotic pressure generated inside the gel network will break the structure of hydrogel by shedding off the discrete pieces. With this unique feature, relatively small amount of the carbopol is required to formulate the hydrophilic matrix tablet.

The factors involved in controlling drug release from hydrophilic matrix tablets were extensively investigated. Drug release from the hydrophilic matrix tablets was less affected by the process variables such as compaction pressure, mixing time and granulation methods $[5,6]$. On the other hand, the formulation variables including drug properties, polymer properties, filler choice and geometric dimension of the matrix were crucial to the drug release from the matrix tablets.

The drug release behavioral from the hydrophilic matrix tablet is a function of drug solubility which was analyzed by the model-independent and model-dependent methods [7]. The study revealed the primary rate limiting factor is matrix erosion for the drugs with poor solubility. When the solubility of the drug is between $0.5 \mathrm{mg} / \mathrm{ml}$ to $5 \mathrm{mg} / \mathrm{ml}$, the drug release rate was controlled by both matrix erosion and medium infiltration into the matrix. In the case of drug solubility more than $5 \mathrm{mg} / \mathrm{ml}$, the rate limiting factor was only the infiltration of medium to the matrix but not the drug solubility. In general, the primary release mechanism of water soluble drug is diffusion and the erosion is the major release mechanism for water insoluble drug. The drugs with high water solubility always exhibit fast release and the rapid initial release so called burst release due to the rapid surface drug diffusion. It is a challenge for formulating the highly water soluble drugs with high dose using the hydrophilic matrix tablet.

In order to obtain a complete gel formation and prolonged drug release, certain polymer concentration should be used in the matrix tablet. Utilizing HPMC as an example, $20 \%-50 \%$ is the typical usage level. For some of the water soluble drugs, when the polymer concentration reaches to a particular level, future increase of the polymer level would not sequentially suppress the drug release $[8,9]$. The polymers with high viscosity grades typically have a fast hydration rate and are more resistant to dilution and 
erosion. This result a strong and thicken gel layer and slower drug dissolution. The high viscosity polymers are generally recommended for highly water soluble drug since the strong and tortuous gel layer provides more resistance to drug diffusion. The particle size of the hydrophilic polymer also can affect the drug release from the matrix tablet. The hydrophilic polymers with smaller particle size have a rapid hydration rate and quick gel formation, therefore better controlled release properties [2, 10] Some polymer manufactures such as Dow chemical provide specific fine particle grades for controlled release applications. It was reported the hydrophobicity of the polymer also plays a role on the drug release.

Incorporation of filler to matrix tablet can improve the flow properties of the granules and mechanical strength of the tablets. It modifies not only the physical properties, but the filler can also manipulate the drug release from the matrix. It is a common strategy for formulation development of the hydrophilic matrix tablet. Water soluble fillers like polyethylene glycol, lactose can increase drug release, because the water penetration was enhanced and the tortuosity of the gel matrix was decreased after the water soluble filler leached out from the system [5]. The insoluble fillers like dicalcium phosphate and microcrystalline cellulose exhibit a relatively slower release rate. The water swellable filler partially pregelatinized maize starch (Starch 1500) was found to have synergistic interaction with the polymer and prevented the water absorption into the matrix, thus slowing down the drug release $[7,11]$.

Geometric factors, including the shape and size of the tablet were reported to affect the drug release from the matrix tablet. The surface area to volume ratio $(\mathrm{S} / \mathrm{V})$ was found to be one of the key variables. The tablets with large $\mathrm{S} / \mathrm{V}$ exhibit fast drug release rate since more surface is available for drug diffusion. The similar release profile can be obtained from the tablets with same $\mathrm{S} / \mathrm{V}$, regardless the tablet shape and dose $[12,13]$. For hydrophilic matrix tablet, the geometry of the tablet keeps changing and the $\mathrm{S} / \mathrm{V}$ is not constant during dissolution. This results in the variation of the drug release rate at different time points. Modification of the geometry of the tablets to maintain the constant $\mathrm{S} / \mathrm{V}$ during dissolution was approved to be effective to achieve the constant release rate. This modification includes the Geomatrix ${ }^{\circledR}$ technology (multi-layer tablet), Smartrix ${ }^{\circledR}$ technology (triple-layered tablet with specific shape in core layer), Dome Matrix ${ }^{\circledR}$ technology (tablet structure arranged by different elementary modules), cup-in-core device and Donald shape tablets. However, many of these designs are very complicated and difficult to manufacture [14].

The combination of a hydrophilic polymer with another hydrophilic or hydrophobic polymer to achieve better drug release performance was extensively investigated. The decreased burst release was observed when a hydrophobic polymer was incorporated into the hydrophilic matrix. This was explained by the increase in the hydrophobicity of the tablet surface and the decrease of water penetration into matrix [15, 16]. However, when a higher ratio of hydrophobic polymer was present in the matrix, it disrupted gel strength and faster drug dissolution occurred [17-19]. The interaction between the nonionic polymer HPMC and anionic polymer $\mathrm{NaCMC}$ helps to overcome the burst effect and lag time associated with the individual polymers. A linear release 
profile can be achieved by use of this combination [20,21]. Combination of the nonionic polymer with the anionic polymer was also utilized to obtain a $\mathrm{pH}$ independent profile for basic drugs. The strong interaction of the basic drug and the anionic polymer leads to the formation of an insoluble drug-polymer complex. This complex retarded the drug release from the matrix and minimized the $\mathrm{pH}$ effect $[22,23]$. The similar phenomenon was appeared while using the combination of nonionic polymer with a cationic polymer and an anionic polymer with a cationic polymer [24-26].

In general, development of the hydrophilic matrix tablets is the state of the art technique. Many formulation factors involved in the development need to be taken into consideration in order to obtain the desired drug release profile and ideal in-vivo performance.

\subsubsection{Hydrophobic matrix tablet}

The primary rate limiting components in a hydrophobic matrix tablet are water insoluble polymers, waxes or combination of both. The examples of hydrophobic waxes material include glycerides, fatty acids and alcohols, hydrogenated vegetable oils [17, 27, 28]. The commonly used hydrophobic polymers consist of ethyl cellulose (EC), cellulose acetate $(\mathrm{CA})$, methacrylic acid copolymers (Eudragit $\left.{ }^{\circledR}\right)$, starch acetates and poly vinyl acetate (PVA) [29-34].

In hydrophobic matrix tablets, the drug is mixed with the hydrophobic components and other excipients, and then be compressed into a tablet. Sustained release profile was achieved by the drug diffusion through the water filled capillary channels or pores existing in tablet network. The drug release behaviors directly related to the porosity and pore structure from the matrix [35]. Such type of matrix is inert in the presence of water and gastrointestinal fluid, and maintains its size and dimensions during drug dissolution. The liquid penetration through the matrix network is the rate-controlling step. The typical mechanism of drug release is diffusion. The drug release from the hydrophobic matrix tablet is a proportional to the square root of time and can be described by the Higuchi equation [36].

The formulation factors and process factors which affect the drug release from the hydrophobic matrix tablets were also investigated by many scientists. The drug properties, particle size of the polymers, formulation variables and process variables were critical factors of drug release from the hydrophobic matrix tablets.

Neau et al [37] studied the drug solubility effect on drug release mechanism from ethylcellulose matrix tablets. Increased drug release rate with an increase of drug solubility was observed for formulations with different drug loadings. At low drug loading, the contribution of polymer relaxation to the drug release diminishes with decreasing drug solubility. Drug solubility effect on drug release was also found in cellulose acetate matrix tablet [38]. The sustained release profile was only obtained for the sparingly water soluble drug theophylline, but not for highly soluble drug dyphylline 
and proxyphylline when cellulose acetate was used as the retarding agent. Additional barrier-coating was required for the highly soluble drug formulations to prevent the massive release and matrix erosion.

The particle size of the polymer is a very important factor in controlling the drug release from the hydrophobic matrix tablet. The coarse grade of hydrophobic polymers failed to achieve the sustained drug release profile while the same viscosity grade of fine particles can effectively control the drug release [39]. According to the percolation theory, fewer drug clusters can be formed when more polymer particles surrounded the drug. Since more polymer particles were presented in the fine particle grades, slower drug release can be obtained [40].

Dabbagh et al [41] investigated the effect of polymer concentration, compression pressure and hydrophilic polymers on drug release from hydrophobic ethylcellulose matrix tablets. The results demonstrated the influence of polymer concentration on drug release rate. Decreased drug release rate was observed as the polymer concentration increased in the matrix, however the polymer concentration did not alter the drug release mechanism. The drug release rate was also affected by the compression pressure. The drug release rate was decreased as the compression pressure was increased to $39.4 \mathrm{MNm}^{-}$ 2 initially. But as the compression pressure reached to the range of $78.7-393.7 \mathrm{MNm}^{-2}$, the drug release was relatively unaffected owing to the elasticity of the ethylcellulose. Drug release rate was increased by incorporation of hydrophilic polymer NaCMC or HPMC $\mathrm{K} 4 \mathrm{M}$ to the hydrophobic matrix. Rapid water uptake and discontinuous gel formation were responsible for the fast drug release profile.

Sadeghi et al [42] found the tensile strength of the matrix tablets was a critical factor for drug release. The results showed the matrices prepared by the water soluble drug propranolol hydrochloride and Eudragit RL or RS could not maintain the physical dimension for a longer period of time. Addition of Aerosil® 200 increased tensile strength of matrices and the tablets could retain their structure which decreased the drug release rate. Azarmi et al [29] reported a thermal treating method to modulate the drug release from Eudragit ${ }^{\circledR}$ RS and Eudragit ${ }^{\circledR}$ RL matrices. When the matrix tablets were heat treated above the glass transition temperature of the polymers, decreased porosity was observed and slower drug release was achieved from the Eudragit matrices.

Compared to the hydrophilic matrix tablets, hydrophobic matrix tablets provided several advantages [17, 19]: a) suitable for highly water soluble drugs; b) drug release is not influenced by the $\mathrm{pH}$ and ionic strength of dissolution medium; c) good stability at varies moisture levels during storage. The major disadvantage of the hydrophobic matrix tablet is the incomplete release of water insoluble drug during the gastrointestinal transition time due to the insufficient concentration gradient [43]. To modulate drug release from this type of matrix, soluble ingredients such as lactose and PEG are required to incorporate into the formulation. The presence of soluble ingredient in the formulation helps to form numerous pores in the hydrophobic matrix network for water penetration and drug diffusion [44]. 


\subsection{Mathematic Models for Matrix System}

\subsubsection{Higuchi equation}

In 1961, Higuchi published a mathematical equation to describe drug release from matrix systems [11]. Although it was developed to describe the drug release profile from the ointment initially, it was later modified to include different geometries and matrix characteristics for describing the drug release from matrix tablets [36]. The basic equation of Higuchi model is Equation 1-1:

$$
\mathrm{Q}_{\mathrm{t}}=\left[\frac{\mathrm{S}^{2} \mathrm{D} \varepsilon}{\tau}\left(2 \mathrm{C}_{0}-\varepsilon \mathrm{C}_{\mathrm{S}}\right) \mathrm{C}_{\mathrm{S}} \mathrm{t}\right]^{\frac{1}{2}}=\mathrm{kt}^{\frac{1}{2}}
$$

where Qt is the cumulative amount of drug released in time $t ; \mathrm{S}$ is the surface area of the tablet; D denotes the drug diffusion coefficient in the matrix phase; Cs is the drug solubility in the matrix; $\mathrm{C}_{0}$ represents the drug concentration in the matrix; $\varepsilon$ is the porosity; $\tau$ denotes the tortuosity of the matrix; and $\mathrm{k}$ is the dissolution rate constant.

Based on this equation, drug release at any given time is proportional to the square root of the time. The release rate constant $\mathrm{k}$ are affected not only by the diffusion coefficient, surface area and drug solubility in the matrix, but also by the matrix properties porosity and tortuosity.

This equation was derived under pseudo steady state assumptions. The assumptions include:

a) Particle size of the drug are small compared to the average diffusion distance;

b) The diffusion coefficient is constant during the dissolution process;

c) Perfect sink conditions are maintained during the dissolution process;

d) The only driving force is the diffusion;

e) The drug concentration in the matrix is greater than the drug solubility in the dissolution medium;

f) There is no interaction between drug and matrix, the swelling or dissolution of the polymer carrier must be negligible; and

g) Only one dimensional diffusion is taken into consideration for the equation, the edge effects must be negligible.

Because of the assumption of the steady state diffusion, the Higuchi equation only can be used to predict drug release less than $70 \%$. If the percentage of drug release is greater than $70 \%$, Higuchi equation cannot give an accurate prediction.

The Higuchi equation is a simple and applicable equation in many cases not only for hydrophobic matrix tablet but also in hydrophilic matrix tablet. When it applied for 
hydrophilic matrix tablet, it is only an empirical equation with square root rate constant and could not characterize the system.

\subsubsection{Power law equation}

The power law equation is a semi-empirical equation to describe the drug release from polymeric systems. The general equation is $[45,46]$ :

$$
\mathrm{M}_{\mathrm{t}} / \mathrm{M}_{\infty}=\mathrm{k}^{*} \mathrm{t}^{\mathrm{n}}
$$

where $\mathrm{M}_{t}$ is cumulative amount of drug released at time $t, \mathrm{M}_{\infty}$ is cumulative amount of drug released at infinite time; $\mathrm{k}$ is release constant incorporating structural and geometric characteristics of the system, and $\mathrm{n}$ is the release exponent indicating the mechanism of drug release.

This equation is derived by Peppas and co-workers, so it is also called Peppas equation. It is a generalization of two independent drug release mechanisms. There are Fickian diffusion and Case II transport. In most systems containing swellable polymers, the drug release is additive result of polymer relaxation and drug diffusion.

The release exponent ' $n$ ' in the power law takes multiple values based on geometry of the system. Different values of $n$ for slab, cylindrical and spherical geometries and corresponding release mechanisms are listed in Table 1-1.

Take the slab as an example, if the $\mathrm{n}$ values $<0.5$, the main mechanism is Fickian diffusion; if the $n$ value $=1$, Case-II transport which is the polymer relaxation is the rate limiting process; if $\mathrm{n}$ value between 0.5 and 1 , the "anomalous transport" takes place where is the combination of both drug diffusion and swelling of the polymer.

The power law equation is a very simple and useful tool to determine the drug release mechanism from the matrix system. It has been successfully applied to many matrix systems $[47,48]$. Similar as the Higuchi equation, the power law equation is only valid for the first $70 \%$ of the release profile. However, it is too simple to offer a robust prediction for complicated release phenomena. These empirical models can only predict the release profile after certain release experiments are conducted and have limited capability to predict how the release profiles will change as the chemical or network properties of the system are varied.

Scientists have also made some modifications to expand the use of the equation. A modification of the power law equation was made by incorporating the concept of lag time [49].

$$
M_{(t-t l a g} / M_{\infty}=k^{*}\left(t-t_{\text {lag }}\right)^{n}
$$


Table 1-1. Release exponent "n" for devices with different geometry

\begin{tabular}{llll}
\hline Slab & Cylinder & Sphere & Release mechanism \\
\hline$<0.5$ & $<0.45$ & $<0.43$ & Fickian diffusion \\
$0.5-1$ & $0.45-0.89$ & $0.43-0.85$ & Anomalous transport \\
$>1$ & $>0.89$ & $>0.85$ & Case transport \\
\hline
\end{tabular}


Another modification was performed by decoupling diffusion and "Case-II transport" with the following expression [50]:

$$
\mathrm{M}_{\mathrm{t}} / \mathrm{M}_{\infty}=\mathrm{k}_{1} * \mathrm{t}^{\mathrm{m}}+\mathrm{k}_{2} * \mathrm{t}^{2 \mathrm{~m}}
$$

where $\mathrm{k}_{1}, \mathrm{k}_{2}$ and $\mathrm{m}$ are constants. First term in the right hand of the equation represents the contribution of Fickian diffusion (F) to overall drug release and the second term represents the contribution of tablet structure relaxation $(\mathrm{R}) . \mathrm{R} / \mathrm{F}$ ratio can be calculated using Equation 1-5.

$$
\mathrm{R} / \mathrm{F}=\mathrm{k}_{2} * \mathrm{t}^{\mathrm{m}} / \mathrm{k}_{1}
$$

The dominant drug release mechanism can be determined by the $\mathrm{R} / \mathrm{F}$ ratio at any given time during dissolution. The drug release profile can be modulated based on the release mechanism.

\subsubsection{Hopfenberg model}

The Hopfenberg model is developed to describe drug release from the degradable or erodible drug delivery system $[51,52]$. The general equation is:

$$
\frac{\mathrm{M}_{\mathrm{t}}}{\mathrm{M}_{\infty}}=1-\left(1-\frac{\mathrm{k}_{0} \mathrm{t}}{\mathrm{C}_{0} \mathrm{a}}\right)^{\mathrm{n}}
$$

where $M_{t}$ and $M_{\infty}$ are the cumulative absolute amounts of drug released at time $t$ and at infinite respectively; $\mathrm{k}_{0}$ is zero order rate constant; $\mathrm{C}_{0}$ denotes the uniform initial drug concentration within the system; and a is the radius of a cylinder or sphere or the halfthickness of a slab; $n$ is a shape factor representing spherical $(n=3)$, cylindrical $(n=2)$ or slab geometry $(\mathrm{n}=1)$.

The model was developed by the following assumptions:

a) There is no edge and end effect.

b) The rate-limiting step of drug release is the surface erosion.

c) Time dependent internal or external diffusion resistances do not influence matrix erosion.

d) Overall kinetics is a zero-order process.

The Hopfenberg model can be applied to a surface eroding polymer matrix system with a zero order surface drug detachment. The drug release rate is proportional to the surface erosion of the device. 


\subsection{Cellulose Acetate (CA)}

\subsubsection{Chemical structure}

Cellulose acetate (CA) is the acetate ester of cellulose. It was produced by the reaction of acetic acid and acetic anhydride with purified cellulose in the present of sulfuric acid. A further partial hydrolysis was used to remove sulfate and a certain number of acetate groups under the controlled conditions. All of the three hydroxyl groups located on the repeated unit of cellulose structure can react and form acetate esters. The amount of esterfication can be controlled by the reaction conditions. The chemical structure of CA is illustrated in Figure 1-1. It contains a cellulose backbone which is a repeat unit of anhydrous glucose and connected by $\beta-1,4$ glycosidic linkages. The three hydroxyl groups were partially substituted by the acetyl group. The degree of the substitution was expressed as the average number of acetyl group in one repeat unit. The length of the cellulose chain and the degree of acetyl substitution directly affect the physicochemical properties of CA.

\subsubsection{Physicochemical properties of CA}

Cellulose acetate is a tasteless, odorless white powder or pellet. It is generally regarded as a nontoxic and nonirritant material. It is hydrophobic in nature and only soluble in certain organic solvents such as acetone, methylene chloride-alcohol blends, methyl acetate, dimethyl formamide, and dioxane. The solubility of CA is greatly affected by the molecular weight and degree of substitution.

There are various grades of CA available on the market with different molecular weight and degree of substitution as listed in Table 1-2. CA polymers have a relatively high glass transition temperature $170-190^{\circ} \mathrm{C}$ and high melting point $230-300^{\circ} \mathrm{C}$. The glass transition temperature and melting point were increased as increasing the molecular weight and degree of substitution of CA. The viscosity of CA polymers listed in Table 1-2 was measured in $10 \% \mathrm{w} / \mathrm{v}$ organic solutions. The viscosity of CA polymers is directly related to the molecular weight and range from 10 to $230 \mathrm{mPa} . \mathrm{s}(10-230 \mathrm{cP})$. Even though there are various grades of CA available, only two of them were approved by FDA for pharmaceutical application. There are CA 320S and CA 398-10 NF.

CA polymers were supplied as fine powder or pellet form. For the powder form, the typical bulk density is $0.32 \mathrm{~g} / \mathrm{cm}^{3}$ and typical tap density is $0.4 \mathrm{~g} / \mathrm{cm}^{3}$. More than $90 \%$ of the powder can pass through 80 mesh sieve. These properties make the CA powder suitable for direct compression application. The pellet form has to be dissolved in a solvent, then form a film after solvent is evaporated. 


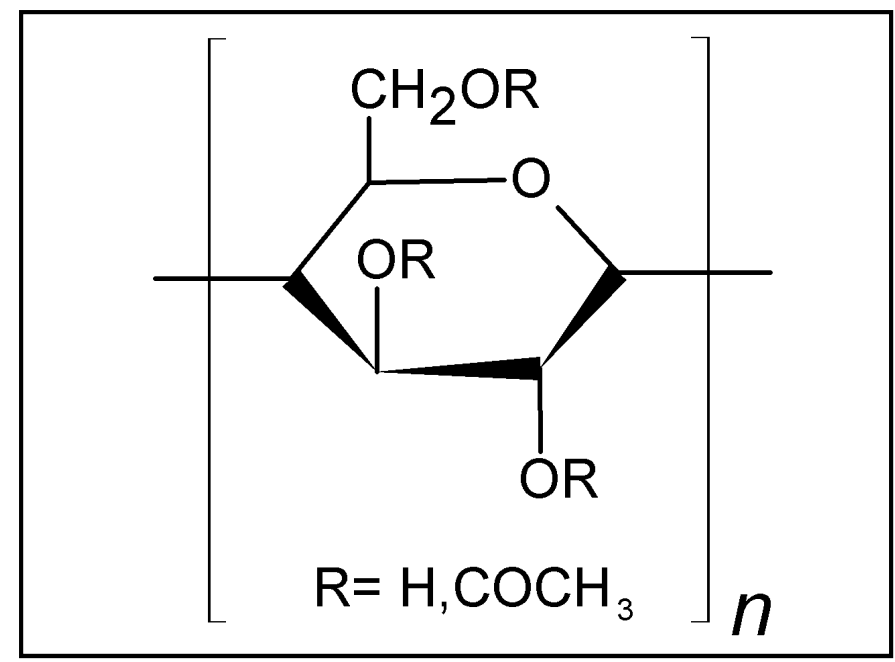

Figure 1-1. Chemical structure of cellulose acetate

Table 1-2. Physical and chemical properties of different grade of cellulose acetate

\begin{tabular}{lccccccc}
\hline CA grades & $\begin{array}{c}\text { Viscosity }^{\mathbf{a}} \\
\mathbf{( c P )}\end{array}$ & $\begin{array}{c}\text { Acetyl } \\
(\mathbf{\%})\end{array}$ & \multicolumn{2}{c}{$\begin{array}{c}\text { Degree of } \\
\text { substitution }\end{array}$} & $\begin{array}{c}\text { Hydroxyl Melting } \\
(\%)\end{array}$ & $\mathbf{T g}^{\circ} \mathbf{C}$ & $\mathbf{M W}_{\mathbf{n}}^{\mathbf{b}}$ \\
& 2.4 & 32.0 & 1.8 & 8.7 & $230-250$ & 180 & 38,000 \\
CA-320S & 11.4 & 39.8 & 2.4 & 3.5 & $230-250$ & 180 & 30,000 \\
CA-398-3 & 22.8 & 39.8 & 2.4 & 3.5 & $230-250$ & 182 & 35,000 \\
CA-398-6 & 38.0 & 39.8 & 2.4 & 3.5 & $230-250$ & 185 & 40,000 \\
CA-398-10NF & 114.0 & 39.7 & 2.4 & 3.5 & $230-250$ & 189 & 50,000 \\
CA-398-30 & 228.0 & 39.5 & 2.4 & 4.0 & $240-260$ & 186 & 60,000 \\
CA-394-60S & N/A & 43.5 & 2.9 & 0.9 & $280-300$ & 185 & 122,000 \\
CA-435-75S & N &
\end{tabular}

${ }^{\mathrm{a}}$ ASTM D 871(Formula A) and D 1343

${ }^{b}$ Number average molecular weight in polystyrene equivalents 


\subsubsection{Pharmaceutical application of CA}

\subsubsection{Osmotic drug delivery system}

The osmotic drug delivery system is a novel delivery system developed in the 1970s which utilizes osmotic pressure as an energy source to control the drug release. It typically consists of the tablet core and a semi-permeable coating layer with a laser drilled hole on the coating membrane. The drug release from the osmotic delivery system was controlled by the water flux rate, drug solubility and osmotic pressure which generated by the osmogent. There are several types of osmotic delivery device on the market including: elementary osmotic pump, push-pull osmotic pump, sandwiched osmotic pump, liquid oral osmotic system, controlled porosity osmotic pump and asymmetric osmotic system. The semi-permeable membrane is an essential part in an osmotic delivery system. This membrane should be insoluble and permeable to the water to pertain sufficient water flux, but relatively impermeable to the contents inside the device to prevent the diffusion of drug and osmogent through the film. It has to be stable and rigid enough to retain its integrity during drug dissolution.

$\mathrm{CA}$ is the most widely used polymer to form the membrane in osmotic drug delivery system due to its excellent mechanical and semi-permeable properties. The properties of the membrane are directly related to the water flux rate and therefore affect the drug release rate. CA membrane has a relatively high water permeability compared to other polymer membranes. The water permeability can be easily be adjusted by the acetyl content of the CA, incorporation of plasticizer and the thickness of the membrane.

As the acetyl content increases, the permeability of CA membrane decreases and water flux rate also decreases. Yuan et al [53] studied an osmotic pump tablet of theophylline by using CA $320 \mathrm{~S}$ or CA $398-10 \mathrm{NF}$ as a coating membrane. The tablets coated with low acetyl content CA (CA-320S) showed higher water permeability and faster release rate of theophylline.

Studies have shown the type and the level of plasticizers can alter the properties of CA film. In general, incorporation of plasticizer to the CA film lowers the glass transition temperature, increases flexibility of film and decreases the strength of the film. The water permeability of the CA film depends on the type of the plasticizer used in the system. Usually, soluble plasticizers increase the water permeability and insoluble plasticizers decrease the water permeability. Guo [54] reported the effect of water soluble plasticizer PEG 600 on water permeability and physical properties of CA film. The water permeability of CA films decreased as increasing PEG-600 to a minimum level due to the antiplasticization effect. At higher concentration PEG 600, especially when the PEG 600 is over $30 \%(\mathrm{w} / \mathrm{w})$, the dramatic increase of water permeability was found owing to the plasticizer channels were formed in the CA films. Liu et al [55] studied the effect of the nature of the plasticizers on the film properties as well as the drug release rate. Addition of hydrophilic plasticizer PEG 200 improved the drug release whereas addition of hydrophobic plasticizer triacetin suppressed the drug release from a monolithic osmotic 
tablet. Films containing PEG as plasticizer developed numerous pores after 24 hours dissolution whereas films containing triacetin as plasticizer still remained a dense film structure.

The thickness of the coating membrane is another factor that affects the drug release from the osmotic system. The water permeability as well as the drug release rate is typically inversely proportional to the thickness of the coating membrane. A study [56] on nifedipine monolithic osmotic tablet found the drug release rate decreases as the thickness of membrane increased from $85-340 \mu \mathrm{m}$. But for the asymmetric osmotic system, the thickness of the coating membrane was found to have a slight effect on drug release rate. This is due to the existence of large numbers of open pores in the membrane. The structure of the membrane played a more important role than the thickness [57].

\subsubsection{Sustained release matrix system}

As a water insoluble hydrophobic polymer, CA is widely used in a sustained release matrix system. Sustained release matrix tablets prepared by cellulose acetate have been investigated by using direct compression or wet granulation methods [38, 58-60]. In most of the cellulose acetate matrix tablet formulations, additional binder was added to improve the tensile strength of the tablets. The results showed drug solubility is an especially important influence on the release rate. The CA matrix is capable of controlling the release rate for a sparingly water soluble drug theophylline when a high concentration of CA were presented in the tablet. But the satisfactory sustained release profiles were difficult to obtain when the drug solubility is higher. This is due to insufficient surrounding of CA to highly soluble drug particles. Additional barriercoating to the soluble drug particles can effectively prevent the massive drug release and matrix erosion.

Another study [61] showed the longer compression dwell time is helpful to prolong the drug release from the CA matrix. In order to keep integration of the matrix tablet during dissolution, enough bonding caused by the fusion between the polymer particles are required. The polymer fusion takes place whiles a temperature higher than glass transition temperature of the polymer occurs during tablet compression. For CA polymers with high glass transition temperature, a long dwell time and high compression pressure are necessary to induce the fusion between the adjacent CA particles. It was concluded that the longer dwell time $(>20 \mathrm{~s})$ of the compression process was required to form bonding by fusion to obtain the sustained drug release profile. But this method is not practical for high speed tablet press production. The production rate will be very limited if a long compression dwell time is applied.

Yuan et al [58] studied the formulation effect on the drug release from the cellulose acetate (CA) or cellulose acetate butyrate (CAB) matrix tablet prepared by direct compression process. It was concluded drug solubility, polymer concentration, type and degree of substitution of polymer, and other excipients presented in the matrix tablets are the key formulation factors. It was found that the tablet prepared by the more 
hydrophobic polymer $\mathrm{CAB}$ showed slower drug release rate than $\mathrm{CA}$. This research also found admixing the plasticizer triethyl citrate (TEC) with CA can help to retard drug release from the matrix tablet.

CA is also used for preparation of matrix microspheres [62-64]. The low bulk density and hydrophobic nature make CA suitable in preparation of a gastric floating system. The smooth and spherical microspheres can be obtained by using CA as a carrier material with encapsulation efficiency ranging from $73 \%$ to $98 \%$. The microspheres sustained the drug release and remained floating for over 10 hours. The drug release rate from the microsphere decreased as the mean particle size and the concentration of the polymer increased [63].

\subsubsection{Other applications}

As an odorless and tasteless polymer with excellent film properties, CA is also used for the taste masking application $[65,66]$. When CA based coating was applied on the surface of acetaminophen granules, the unpleasant bitter taste of acetaminophen diminished. A taste masked chewable tablet can be obtained by compressing these granules with other excipients. Yu et al [66]coated the levofloxacin particles by using the combination of $\mathrm{CA}$ and other $\mathrm{pH}$ sensitive polymer Eudragit ${ }^{\circledR} \mathrm{E}-100$. The improved palatability of drug was obtained. These taste masked drug particles were suitable for oral liquid dosage form.

CA film can be used as rate controlling membrane for transdermal delivery system $[47,67]$. The smooth and flexible film can be prepared by using CA and plasticizer. The plasticized films are permeable for both water vapor and drug. The permeability coefficient of the drug is dependent on the type of plasticizer and the thickness of the film. High permeability coefficient was observed when water soluble plasticizer was applied. Drug diffusion rate increased as thickness of the film decreased. The drug diffusion from the film can be prolonged and followed zero order kinetics. Incorporation of water soluble polymer PVP into the CA film decreased the tensile strength of the film and increased film permeability, but did not alter the drug diffusion kinetics.

\subsection{Application of Plasticizers on Oral Solid Dosage Form}

Plasticizers are typically low molecular weight compounds which are capable of penetrating into the polymer structure and making them soft and flexible. The process of adding a plasticizer or heat to soften the material and makes it more plastic is called plasticization. The plasticization was applied in several of pharmaceutical process including film preparation, hot melt extrusion and matrix tablet preparation to improve the process condition or/and achieve better functionality.

The plasticization is occurred by the intermolecular interaction between the 
plasticizer and the polymer. This interaction increases the free volume between the polymer chains and ease of movement of polymer chains could be dramatically increased. Thus both of the glass transition temperature and the melting viscosity of the polymer decreases after plasticization. The interaction of polymer and plasticizer also help to lower the melting energy of the semi-crystalline polymers and facilitate the fusion.

The purpose of incorporation of plasticizer into the film formulation is to improve the mechanical properties and modify drug release behavior. The plasticizer typically increases the elongation and elastic modulus of film. Addition of plasticizer can prompt to film formation, reduce the brittleness, improve the flexibility and plasticity and increase the mechanic resistance of the film. Lower water permeability and oxygen transition rates were observed for plasticized film. The drug release rate from the film or the film coated pellets can be modulated by the addition of the plasticizer. In general, hydrophilic plasticizer accelerates the drug release and hydrophobic plasticizer suppresses the drug release.

Application of the plasticizer in the hot-melt extrusion process often lowers the process temperature and improves the process condition. With the help of the plasticizer, a hot-melt extrusion process can be performed at lower temperatures to avoid the decomposition of the drug and the polymer. Since the decreased viscosity of the polymer can be obtained after addition of the plasticizer, less energy is required for the process and the process condition is improved.

The application of the plasticizer in sustained release tablets has been reported by several scientists. Hardy et al [68] studied the plasticizer effect on the compression properties of an HPMC matrix tablet. Except for water soluble plasticizer propylene glycol, addition of other plasticizers especially hydrophobic plasticizer decreased the tensile strength of the tablets and increased elasticity of the tablets. Yuan et al [58] admixed the plasticizer with CA to modify theophylline release from the hydrophobic matrix tablet. The rate of release of theophylline decreased after addition of the plasticizer TEC. Xiao [69] investigated the plasticizer effect on the sustained release matrix tablets prepared by ethyl cellulose. It was found addition of the plasticizer decreased porosity of the tablet, thus the drug release rate was also decreased. The slower drug release rate was observed for the tablets prepared with more concentrated and hydrophobic plasticizer.

As a plasticizer for pharmaceutical application, the following properties are required:
a) Good plasticization efficiency
b) Non toxic
b) Compatible with drug and polymer
d) Nonvolatile and stable in the storage condition
e) Regulatory acceptance 
The commonly used pharmaceutical plasticizers and their physicochemical properties are listed in Table 1-3. They have been investigated as plasticizers in different pharmaceutical processes to demonstrate a good interaction with the polymers and a good safety profile.

\subsection{Co-processed Excipients for Oral Solid Dosage Forms}

\subsubsection{Excipient for oral solid dosage form}

All the oral solid dosage forms consist of active pharmaceutical ingredients (API) and excipients. Historically, excipients has been viewed as merely inert additives and not been given the credit for their effect on the finished pharmaceutical dosage form. But the importance of the excipients has been realized in recent years. In fact, the fate of a drug product can be determined by the functionality of the excipient. The major contributions of excipient to the end product include: 1) improving the process condition during manufacturing [70]; 2) improving the physicochemical properties of API and enhancing the stability and bioavailability; 3) modulating the drug release characteristic in the body and improve the patient acceptability; 4) facilitating administration; 5) aiding product identification; 6) attributing to overall safety and effectiveness.

The tablet is the most preferred dosage form among all oral solid dosage forms. This is due to the precise dosing, cost effective manufacturing and good patient compliance. The principal excipients in a tablet formulation include filler, binder, disintegrant, lubricant, glidant and coating material. In-depth knowledge of physicochemical properties of excipients and numerous pharmaceutical tests are required while selecting the right excipient for a tablet formulation. Excipients with multiple functions leading to less complicated formulation as well as time and cost effectiveness are more preferred to be selected by pharmaceutical formulators.

Tablets are manufactured by either granulation or direct compression. Direct compression is simpler and less expensive process because multiple granulation processes were avoided. But it has specific requirement for the properties of the raw materials such as good flowability, good compactability, less segregation potential, low lubricant sensitivity and good machineability in a high speed tablet press. For most of the tablet formulations (70-80\%), the concentration of the excipients are higher than the active drug. Therefore, excipients play a major role for direct compression formulations $[6,71]$. In order to reduce the development process and get the product to the market faster, there is an increasing demand for multi-functional excipients for direct compression formulations. 
Table 1-3. Physical and chemical properties of commonly used pharmaceutical plasticizers

\begin{tabular}{lccc}
\hline Plasticizers & $\begin{array}{c}\text { Boiling point } \\
\left(\mathbf{(}^{\mathbf{C}}\right)\end{array}$ & $\begin{array}{c}\text { Water solubility } \\
(\mathbf{g} / \mathbf{1 0 0} \mathbf{~ m l}) @ \mathbf{2 5}^{\mathbf{0}} \mathbf{C}\end{array}$ & Log P \\
\hline Triethyl Citrate (TEC) & 288 & 5.5 & $1.493 \pm 0.570$ \\
Acetyltriethyl Citrate (ATEC) & 294 & 0.7 & $3.731 \pm 0.641$ \\
Tributyl Citrate (TBC) & 322 & $<0.1$ & $4.681 \pm 0.571$ \\
Acetyltributyl Citrate (ATBC) & 326 & $<0.1$ & $6.919 \pm 0.641$ \\
Dibutyl Sebacate (DBS) & 345 & $<0.1$ & $5.975 \pm 0.228$ \\
\hline
\end{tabular}




\subsubsection{The source of new excipient}

The improved functional excipients typically come from new chemical entities, new grades of existing materials or a new combination of existing materials [10, 72]. Development of a new chemical entity is usually a high risk and large investment. It must undergo a very long cycle with various stages of regulatory approval for assessment of safety and toxicity $[10,71]$. The approval of new excipients is even more complicated than a new drug product. It is a very time and money consuming process. Successful new grades of the existing excipients can be obtained by the chemical modification such as the pregelatinized starch, croscarmellose, and crospovidone, or by particle engineering such as spray dried lactose, Avicel $® 101,102,200$ (Microcrystalline cellulose), Methocel ${ }^{\circledR}$ DC (direct compressible HPMC). It has been a very successful approach for developing new excipients over last three decades. But the extent of functional improvement is very limited because of the restricted range of possible modification. A new combination of the existing excipients is another good choice because most tablet formulations currently consist of multiple excipients. A large number of excipient combinations are available for improving the performance of the existing excipients. Two development processes of the new combinations are typically involved. They are physical mixing and co-processing. The most successful physically mixed excipient on the market is Opadry®. It is a preblended dry powder coating formulation. It combines polymer, plasticizer and pigment to provide a one-step coating formula. Kollidon ${ }^{\circledR}$ SR is another example of a combination of mixed excipients. It contains polyvinyl acetate and polyvinyl pyrrolidone at ratio 80:20 and is used for sustained release matrix tablets. The co-processed excipient is combined at a subparticle level of excipients. This is much broader platform for improving the functionality of the existing excipients and has gained more attention over the years.

\subsubsection{Co-processed excipient}

The International Pharmaceutical Excipients Council (IPEC) defines a coprocessed excipient as follows: "A co-processed excipient is a combination of two or more compendial or non-compendial excipients designed to physically modify their properties in a manner not achievable by simple physical mixing and without significant chemical change" [72]. Co-processed excipients contain two or more excipients and possess the performance advantages over the physical mixture. Not only improved performance but also reduction of undesired properties of a single excipient can be achieved by using co-processing. The co-processing excipients are typically produced by the commercially available pharmaceutical manufacture processes, such as wet granulation, dry granulation, spray drying and freeze drying. Spray drying is the most commonly used method and it is very simple and effective. Most importantly, it can incorporate one excipient into another excipients' particle structure and provide desirable physical advantages.

The typical process of developing a co-processed excipient includes the following steps [73]: a) identify the functionality requirements and select the appropriate excipients; b) determine the ratio of various excipients; c) select the suitable co-process method such 
as spray dry, wet granulation; d) optimize the preparation process; e) evaluate the physicochemical properties and functionality of the co-processed excipient.

The particle properties of excipients such as particle size, particle size distribution, powder porosity and surface roughness can be modified by co-processing, thus improve the functionality of the excipients including improved flowability, better compactability and reduced segregation potential. These improved properties of an excipient make it more suitable for direct compression formulations. The major benefits of the excipients received from the co-processing were summarized as [73]: a) lack of chemical change; b) reducing the number of excipients in one formulation; c) improved physical mechanical properties such as flowability and compactability; d) better dilution potential and less segregation potential; e) less lubricant sensitivity.

There are several co-processed excipients for immediate release formulation available on the market as listed in Table 1-4. The improved manufacturing efficiency and reduced cost of the final drug product can be obtained by using these co-processed excipients.

\subsubsection{New excipients in sustained release application}

A sustained release matrix tablet is the simplest and the most cost effective delivery system for modulating the drug release behavior in the body. The market of sustained release tablets keeps increasing every year, but the development of new excipients is relatively slow. There are only two new sustained release excipients coming to the market in the last few decades.

One is Methocel ${ }^{\circledR}$ DC which was developed by Dow Chemical. It is a new grade of HPMC polymers customized for direct compression application. The DC grade of HPMC can achieve better flowability without comprising the sustainability and compactability by strictly controlling the particle size distribution [74]. The other one is Kollidon ${ }^{\circledR}$ SR which developed by BASF. It is a physical mixture of polyvinyl acetate and polyvinyl pyrrolidone at ratio $80: 20$. Kollidon ${ }^{\circledR}$ SR can be used for both granulation and direct compression process. The water soluble components polyvinyl pyrrolidone can gradually leaches out from the matrix during dissolution thereby modulating the drug release from the hydrophobic matrix system [75].

Beside the commercial available excipients, Xiao [69] developed a co-processed excipient consisting of ethyl cellulose, plasticizer and fillers by a wet granulation method. This excipient showed good flowability and compactability and can be used for preparation of a hydrophobic matrix tablet. But this hydrophobic matrix tablet is only suitable for the drugs with low water solubility. For highly water soluble drug, a compression coating has to be applied to obtain a desired in vitro release profile.

In our study, we have developed a co-processed sustained release excipient utilizing a cellulose acetate with plasticizer and other filler via various preparation 
Table 1-4. Commercial available co-processed excipients

\begin{tabular}{|c|c|c|c|}
\hline $\begin{array}{l}\text { Ingredients of } \\
\text { co-processed } \\
\text { excipients }\end{array}$ & Trade name & Manufacturer & Improved functions \\
\hline $\begin{array}{l}\text { Lactose, } \\
\text { Kollidon 30, } \\
\text { Kollidon CL }\end{array}$ & Ludipress & BASF & $\begin{array}{l}\text { Low degree of } \\
\text { hygroscopicity, good } \\
\text { flowability, tablet hardness } \\
\text { independent of machine speed }\end{array}$ \\
\hline $\begin{array}{l}\text { Mannitol, } \\
\text { Kollindon } \\
\text { CL,PVA }\end{array}$ & Ludiflash & BASF & $\begin{array}{l}\text { Less hydroscopicity, directly } \\
\text { compressible, good flowability, } \\
\text { resulting hard tablet with lower } \\
\text { friability }\end{array}$ \\
\hline $\begin{array}{l}\text { Lactose, } \\
\text { Lactitol }\end{array}$ & $\begin{array}{l}\text { Pharmatose } \\
\text { DCL } 40\end{array}$ & DMV & $\begin{array}{l}\text { Good flowability, high dilution } \\
\text { potential, lower water uptake at } \\
\text { high humidity }\end{array}$ \\
\hline $\begin{array}{l}\text { Lactose, } \\
\text { cellulose }\end{array}$ & Cellactose & Meggle & $\begin{array}{l}\text { Highly compressible, good } \\
\text { mouth-feel, better tableting } \\
\text { at low cost }\end{array}$ \\
\hline $\begin{array}{l}\text { Lactose, } \\
\text { MCC }\end{array}$ & Microcelac & Meggle & $\begin{array}{l}\text { Good flowability and binding } \\
\text { properties, less lubricant } \\
\text { sensitivity }\end{array}$ \\
\hline $\begin{array}{l}\text { MCC, } \\
\text { silica dioxide }\end{array}$ & Prosolv & JRS Pharma & $\begin{array}{l}\text { Better flow, tablet strength and } \\
\text { less lubricant sensitivity }\end{array}$ \\
\hline MCC, Mannitol & $\begin{array}{l}\text { Avicel HFE } \\
102\end{array}$ & $\begin{array}{l}\text { FMC } \\
\text { Biopolymer }\end{array}$ & $\begin{array}{l}\text { Better tablettability at slower } \\
\text { tablet speed, less lubricant } \\
\text { sensitivity }\end{array}$ \\
\hline $\begin{array}{l}\text { MCC, Guar } \\
\text { gum }\end{array}$ & $\begin{array}{l}\text { Avicel CE- } \\
15\end{array}$ & $\begin{array}{l}\text { FMC } \\
\text { Biopolymer }\end{array}$ & $\begin{array}{l}\text { Smoother ,creamier mouth feel, } \\
\text { less tooth-packing }\end{array}$ \\
\hline $\mathrm{MCC}, \mathrm{Na} \mathrm{CMC}$ & $\begin{array}{l}\text { Avicel CL- } \\
611\end{array}$ & $\begin{array}{l}\text { FMC } \\
\text { Biopolymer }\end{array}$ & Increase formulation stability \\
\hline Starch, Lactose & StarLac & Roquette & $\begin{array}{l}\text { Good flowabililty and } \\
\text { compactability with rapid } \\
\text { disintegration time }\end{array}$ \\
\hline $\begin{array}{l}\text { Kollindon VA } \\
64, \\
\text { Plasdone S } 630\end{array}$ & Copovidone & BASF & $\begin{array}{l}\text { Good flowability and binding } \\
\text { properties }\end{array}$ \\
\hline Sugar, dextrin & Di-Pac & Domino & $\begin{array}{l}\text { Good flowability, low } \\
\text { hygroscopicity, direct } \\
\text { compressible }\end{array}$ \\
\hline $\begin{array}{l}\text { MCC, } \\
\text { crospovidone, } \\
\text { HPMC }\end{array}$ & $\begin{array}{l}\text { PanExcea } \\
\text { MHC300G }\end{array}$ & Avantor & $\begin{array}{l}\text { Good flowability, compactability } \\
\text { with rapid disintegration for } \\
\text { immediate release tablet }\end{array}$ \\
\hline $\begin{array}{l}\text { Mannitol, } \\
\text { calcium silicate }\end{array}$ & $\begin{array}{l}\text { PanExcea } \\
\text { MHC200G }\end{array}$ & Avantor & $\begin{array}{l}\text { Good flowability, compactability } \\
\text { with rapid disintegration for oral } \\
\text { disintegrating tablet }\end{array}$ \\
\hline
\end{tabular}


methods. This co-processed excipient is designed to be used for directly compressed sustained release matrix formulation and is suitable for both water soluble and insoluble drugs. 


\section{Chapter 2. Preparation of Co-processed Plasticized Cellulose Acetate Excipient and Evaluation of the Physical and Compaction Properties}

\subsection{Introduction}

A significant growth of the oral drug delivery system market was observed over the last decade. According to the GBI's research, it will maintain an $11.3 \%$ annual growth rate till 2016 [76]. A sustained release matrix tablet is one of the major components in the oral drug delivery system. This is the simplest and the most cost effective dosage form for modulating the drug release behavior in the body. The manufacturing process for sustained matrix tablets is similar to that of traditional tablets. The common preparation methods are wet granulation, dry granulation and direct compression. Among these methods, the direct compression method is the simplest and requires less unit processes. But it has specific requirements for the properties of the powder such as flowability, compactability. For most of the tablet formulations (70$80 \%$ ), the concentration of the excipients are higher than the active drug. Therefore, excipients play a major role for direct compression formulation [6,71]. In order to reduce the development process and get the product to the market faster, there is a market demand for multi-functional excipients for direct compression formulation development of sustained release matrix tablets.

The improved functional excipients typically come from new chemical entities, new grades of existing materials or a new combination of existing materials [72]. Development of a new chemical entity is usually a high risk and large investment. It must undergo various stages of regulatory approval for assessment of safety and toxicity [71] which is a time and money consuming process. Successful new grades of the existing excipients can be obtained by the chemical modification such as the pregelatinized starch, croscarmellose, and crospovidone, or by particle engineering such as spray dried lactose, Avicel ${ }^{\circledR} 101,102,200$ (Microcrystalline cellulose), Methocel ${ }^{\circledR}$ DC (direct compressible HPMC). It has been a very successful approach for developing new excipient over last three decades. But the extent of functional improvement is very limited because of the restricted range of possible modification. A new combination of the existing excipients is another good choice because most tablet formulations currently consist of multiple excipients. A large number of excipient combinations are available for improving the performance of the existing excipients. Two development processes of the new combinations are typically involved. They are physical mixing and co-processing. The most successful physically mixed excipient on the market is Opadry ${ }^{\circledR}$. It is a pre-blended dry powder coating formulation. It combines polymer, plasticizer and pigment to provide a one-step coating formula. Kollidon ${ }^{\circledR}$ SR is another example of a combination of mixed excipients. It contains polyvinyl acetate and polyvinyl pyrrolidone at ratio 80:20 and used for sustained release matrix tablets. The co-processed excipient is combined at a subparticle level of excipients. This is much broader platform for improving the functionality of the existing excipients and has gained more attention over the years.

The International Pharmaceutical Excipients Council (IPEC) defines a co- 
processed excipient as follows: "A co-processed excipient is a combination of two or more compendial or non-compendial excipients designed to physically modify their properties in a manner not achievable by simple physical mixing and without significant chemical change". Co-processed excipient contains two or more excipients and possesses the performance advantages over the physical mixture. Not only improved performance but also reduction of undesired properties of a single excipient can be achieved by using co-processing. The co-processing excipients are typically produced by the commercially available pharmaceutical manufacture processes, such as wet granulation, dry granulation, spray drying and freeze drying. Spray drying is the most commonly used method and it is very simple and effective. Most importantly, it can incorporate one excipient into another excipients' particle structure and provide desirable physical advantages.

Several co-processed excipients for immediate release formulation are available on the market. The improved manufacturing efficiency and reduced cost of the final drug product can be obtained by using the co-processed excipient. But there is no commercially available co-processed excipient for sustained release matrix formulation. In our study, we have developed a co-processed sustained release excipient utilizing a hydrophobic polymer with plasticizer and other filler via various preparation methods. This co-processed excipient is designed to be used for directly compressed sustained release matrix formulation.

The hydrophobic polymer we selected in our study is cellulose acetate (CA). It is an extensively used polymer in pharmaceutical applications. Because of the hydrophobic nature and good film properties of the CA, it is potentially a good polymer candidate for sustained release matrix tablets. Sustained release matrix tablets prepared by cellulose acetate have been investigated by using direct compression or wet granulation methods $[58,61]$. The results show that for a sparingly soluble drug theophylline, a large amount of CA was required to achieve the desired sustained release profile. It was difficult to formulate a highly water soluble drug by using CA as the retarding agent. Some studies concluded that CA is very sensitive to the solubility of the drug and it is not suitable for retarding the release for highly water soluble drug.

The purpose of our study is to modify the physical characteristic of the CA by using co-processing technique and increasing the ability of cellulose acetate for controlling the drug release rate for drugs of varying solubility. This chapter is focused on the preparation and physical characterization of this novel co-processed excipient.

\subsection{Materials and Methods}

\subsubsection{Materials}

Cellulose acetate with $39.8 \%$ acetyl content and $38 \mathrm{cp}$ viscosity (CA 398-10 NF, Eastman, Kingsport, TN) was used as the main polymer in this study. Triethyl citrate 
(TEC), acetyltriethyl citrate (ATEC), acetyltributyl citrate (ATBC) and dibutyl sebacate (DBS) (Morflex Inc., Greensboro, NC) were used as plasticizers. Regular dicalcium phosphate dihydrate (DCP, Rhodia Inc., Blue Island, IL) and milled dicalcium phosphate dihydrate (milled DCP Innophos Inc., Cranbury, IL) were used as filler. Magnesium stearate (Mallinckrodt, Paris, KY) was used as a lubricant.

\subsubsection{Methods}

\subsubsection{Co-processed methods}

Three different methods including heat treatment, wet granulation and spray drying were investigated for preparing the co-processed excipients. The formulations of the co-processed excipients were listed in Table 2-1. We selected ATEC as a hydrophilic plasticizer in F1 and ATBC as a hydrophobic plasticizer in F2. For comparison, we also prepared formulation F0 which doesn't contain any plasticizer.

A heat treatment method is the simplest preparation method in this study. CA was mixed with appropriate amount of plasticizer in a mortar and pestle. This mixture was treated in the $100^{\circ} \mathrm{C}$ oven for 12 hours. The heat treated CA and plasticizer mixture was further mixed with regular DCP in a twin shell blender (Patterson-Kelley Co, East Stroudsburg, PA) for 3 minutes.

The wet granulation method was performed in a high shear granulator (Collete MICROGRAL, Niro Inc., Columbia, MD). Appropriate quantities of CA were mixed with the milled DCP at impeller speed $1000 \mathrm{rpm}$ for 1 minute. The plasticizer was dissolved into acetone-isopropyl alcohol (70:30, v/v) solution. The liquid mixture was added to the powder bed by a peristaltic pump at speed of $20 \mathrm{~g} / \mathrm{min}$. The liquid mixed with the powder at impeller speed of $1000 \mathrm{rpm}$ and chopper speed of $4000 \mathrm{rpm}$ for 2 minute to obtain the wet mass. The wet mass was dried in an oven dryer at $60^{\circ} \mathrm{C}$ for 2 hours to remove the solvent. The dried granules were passed through a 20 mesh sieve to remove the large agglomerates.

The spray drying method was performed at lab scaled mini spray dryer B295 (Buchi, Switzerland). CA was dissolved in acetone: isopropyl alcohol (50:50) solution at the concentration of $3 \%$. An appropriate amount of plasticizer was added in this solution. The milled DCP was suspended in the solution using a magnetic stirrer. This suspension was spray dried while stirring. Operation parameters are as following: Inlet temperature: $80^{\circ} \mathrm{C}$; outlet temperature $36-50^{\circ} \mathrm{C}$; aspirator: $60 \%$; air volume: $40 \mathrm{ml}$ and pumping rate: $50 \%$. The resulting powders were kept in vacuum oven at $60{ }^{\circ} \mathrm{C}$ for 12 hours to remove the excess solvent and then compressed into the $13 \mathrm{~mm}$ tablets under the compression force of 0.3 metric ton by using the Carver press. The co-processed granules were obtained by passing the large tablets through a 20 mesh sieve. In order to investigate the effect of formulation factors on the physical properties of the co-processed excipient, the formulations containing different plasticizers and different concentration of plasticizer 
Table 2-1. Formulations of the co-processed excipients for three preparation methods

\begin{tabular}{lccc}
\hline \multirow{2}{*}{ Ingredients } & \multicolumn{3}{c}{ Percentage (\%) } \\
\cline { 2 - 4 } & F0 & F1 & F2 \\
\hline CA 398-10 NF & 20 & 20 & 20 \\
ATBC & 0 & 0 & 10 \\
ATEC & 0 & 10 & 0 \\
DCP & 80 & 70 & 70 \\
Total & 100 & 100 & 100 \\
\hline
\end{tabular}


were prepared by spray dried method and listed in Tables 2-2 and 2-3.

\subsubsection{Bulk density, tap density and Carr's index evaluation}

Fill the dry granules in a $50 \mathrm{ml}$ graduated cylinder to approximately $40 \mathrm{ml}$, record the weight and bulk volume. The cylinder was tapped on a tap device (Vankel Industrails, Edison, NJ) for 100, 200, 300, 400 times until a constant volume was achieved. This volume was recorded as the tap volume. The bulk density $(\mathrm{g} / \mathrm{ml})$ was calculated by Equation 2-1. The tap density was calculated by Equation 2-2 and the Carr's index was calculated by Equation 2-3.

$$
\begin{aligned}
& \text { Bulk density }=\text { Weight of the sample }(\mathrm{g}) / \text { Bulk volume }(\mathrm{ml}) \\
& \text { Tap density }=\text { Weight of the sample }(\mathrm{g}) / \text { Tap volume }(\mathrm{ml}) \\
& \text { Carr's index }=100 *(\text { Tap density-Bulk density }) / \text { Tap density }
\end{aligned}
$$

(Eq. 2-3)

\subsubsection{Powder porosity evaluation}

The true density of the powder was measured by a helium pycnometer (AccuPyc 1330, Micromeritics, Norcross, GA). Powder porosity was calculated from the Equation 2-4.

$$
\text { Powder porosity }(\%)=(1-\text { bulk density/true density })^{*} 100
$$

\subsubsection{Particle size analysis}

The particle size distribution was evaluated by sieve analysis. This experiment was performed on the Gilsonic Autosiever (Gilson Company Inc, Lewis Center, Ohio) for 7 minutes at amplitude of $5 \mathrm{~mm}$. The mesh size of the sieve used in this study were $20(850 \mu \mathrm{m}), 35(500 \mu \mathrm{m}), 60(250 \mu \mathrm{m}), 80(180 \mu \mathrm{m}), 120(125 \mu \mathrm{m}), 140(106 \mu \mathrm{m})$ and $200(75 \mu \mathrm{m})$. Approximately $10 \mathrm{~g}$ of granules was used for analysis. The geometric mean particle size was calculated by Equation 2-5.

$$
\mathrm{D}_{\mathrm{gw}}=\log ^{-1}[(\Sigma(\mathrm{Wi} * \log \mathrm{Di}) / \Sigma \mathrm{Wi}]
$$

where $\mathrm{D}_{\mathrm{gw}}$ is the geometric mean particle size, $\mathrm{W}_{\mathrm{i}}$ is the weight of the granules stayed on the $i$ th sieve, Di is the diameter of the $i$ th sieve in the stack. 
Table 2-2. Formulations of the co-processed excipients prepared from different plasticizers

\begin{tabular}{lccccc}
\hline \multirow{2}{*}{ Ingredients } & \multicolumn{5}{c}{ Percentage (\%) } \\
\cline { 2 - 6 } & F0 & F1 & F2 & F3 & F4 \\
\hline CA 398-10 NF & 20 & 20 & 20 & 20 & 20 \\
ATEC & 0 & 10 & 0 & 0 & 0 \\
ATBC & 0 & 0 & 10 & 0 & 0 \\
TEC & 0 & 0 & 0 & 10 & 0 \\
DBS & 0 & 0 & 0 & 0 & 10 \\
Milled DCP & 70 & 70 & 70 & 70 & 70 \\
Total & 100 & 100 & 100 & 100 & 100 \\
\hline
\end{tabular}

Table 2-3. Formulations of the co-processed excipients prepared from different concentration of plasticizers

\begin{tabular}{lcccc}
\hline \multirow{2}{*}{ Ingredients } & \multicolumn{4}{c}{ Percentage (\%) } \\
\cline { 2 - 5 } & F0 & F2 & F5 & F6 \\
\hline CA 398-10 NF & 20 & 20 & 20 & 20 \\
ATBC & 0 & 10 & 2.5 & 5 \\
Milled DCP & 80 & 70 & 77.5 & 75 \\
Total & 100 & 100 & 100 & 100 \\
\hline
\end{tabular}




\subsubsection{Powder flowability evaluation}

In order to provide an overall evaluation of the powder flowability, a weighted composite index was used [77]. It can be calculated by Equation 2-6. The three parameters (critical orifice, Carr's index and angle of repose) in this equation all contribute to $1 / 3$ of the overall flowability. The highest value of the composite index is 100. If the value is greater than 70, the sample can be considered to have a good flowability. Carr's index was calculated by Equation 2-3. Critical orifice was measured by using a Flowdex (Hanson Research, Chatsworth, CA) and the angle of repose was measured by funnel method.

$$
\begin{aligned}
\text { Composite index }= & -10 / 9 * \text { critical orifice }+340 / 9+(-2 / 3 * \text { Carr's Index }) \\
& +110 / 3+(-2 / 3 * \text { angle of repose })+50
\end{aligned}
$$

\subsubsection{Specific surface area measurement}

Specific surface area of all the batches was determined by the nitrogen gas adsorption method. The weighed sample was first degassed at $40^{\circ} \mathrm{C}$ under nitrogen gas to remove moisture and contamination. The surface area of the degassed sample was measured by the Gemini Surface Analyzer (Micromeritics, Norcross, GA) using nitrogen gas as absorption gas source. The multipoint BET (Brunauer, Emmett and Teller) equation was used for calculation.

\subsubsection{Scanning electron microscopy (SEM) study}

SEM was used to characterize the powder morphology. The powders were first coated by $10 \mathrm{~nm}$ gold on the surface for improving the conductivity, and then were examined by the FEI XL-30 Scanning Electron Microscopy. (FEI, Hillsboro, OR).

\subsubsection{Compactability evaluation}

The compactability of the co-processed excipients was evaluated by the compression profile and the Heckel plot. The co-processed excipient was first mixed with $0.5 \%$ magnesium stearate in a twin shell blender (Patterson-Kelley Co, East Stroudsburg, PA) for 2 minutes, and then was compressed into $400 \mathrm{mg}$ tablets at compression forces of $0.3,0.5,0.8,1.0$, and 1.5 metric ton by using a $3 / 8$ inch flat faced tooling on a Carver press (Carver Inc., Wabash, IN) . The dwell time was maintaining 3 seconds for all the batches. Five tablets were prepared for each batch. Hardness of the resulting tablet was measured by a PTB 311 hardness tester (PharmaTest, Germany). The tensile strength of tablets was calculated from the Equation 2-7. 
where $\mathrm{D}$ is the tablet diameter and 1 is the tablet thickness.

The Heckel's plot $[78,79]$ for the co-processed excipient was constructed by the Equation 2-8.

$$
\ln (1 /(1-\mathrm{D}))=\mathrm{kP}+\mathrm{A}
$$

where $\mathrm{D}$ is the relative density of a powder column at pressure $\mathrm{P}, \mathrm{k}$ is the slope. A is a constant. The slope of the Heckel's equation can be used to calculate the yield strength as Equation 2-9.

$$
\mathrm{Y}=1 / \mathrm{k}
$$

The lower yield strength indicates less amount energy is required for plastic deformation.

\subsubsection{Glass transition temperature (Tg) measurement}

A cast film method was used to prepare film samples. CA 398-10 NF was dissolved into acetone solution at a concentration of 10\%. Appropriate amount of plasticizer was added to the solution and mixed uniformly with the $10 \%$ CA solution. Thereafter, $1 \mathrm{ml}$ of the solution was transferred to a Teflon Petri dish and dried at $60^{\circ} \mathrm{C}$ in vacuum oven for 24 hours to remove the solvent. The Differential Scanning Calorimeters (DSC) (TA instruments, New Castle, Delaware) was used to determine the Tg of the film. Conventional modulate DSC program was employed in this study. The heating rate is 20 ${ }^{\circ} \mathrm{C} / \mathrm{min}$ and the heating range is $30^{\circ} \mathrm{C}$ to $220^{\circ} \mathrm{C}$. The TA Universal V $4.4 \mathrm{~A}$ software was used to analyze the data.

\subsection{Results and Discussion}

\subsubsection{Effect of different process methods}

\subsubsection{Morphology}

The SEM image of the raw material as well as the co-processed excipients was shown in Figure 2-1. Since the images of F0, F1 and F2 for each process method are similar, we only selected F2 as an example. The original CA has the irregular particle shape and the rough surface. After it mixed with the plasticizer ATBC and heated for 12 hours in the oven, as shown in Figure 2-1B, the particle shape and the surface structure remained similar to the original CA. This indicates the plasticizer is only physically adsorbed on the surface of CA or penetrates into the small pores in the CA particles. Strong interaction between the polymer and the plasticizer was not observed. For the wet 


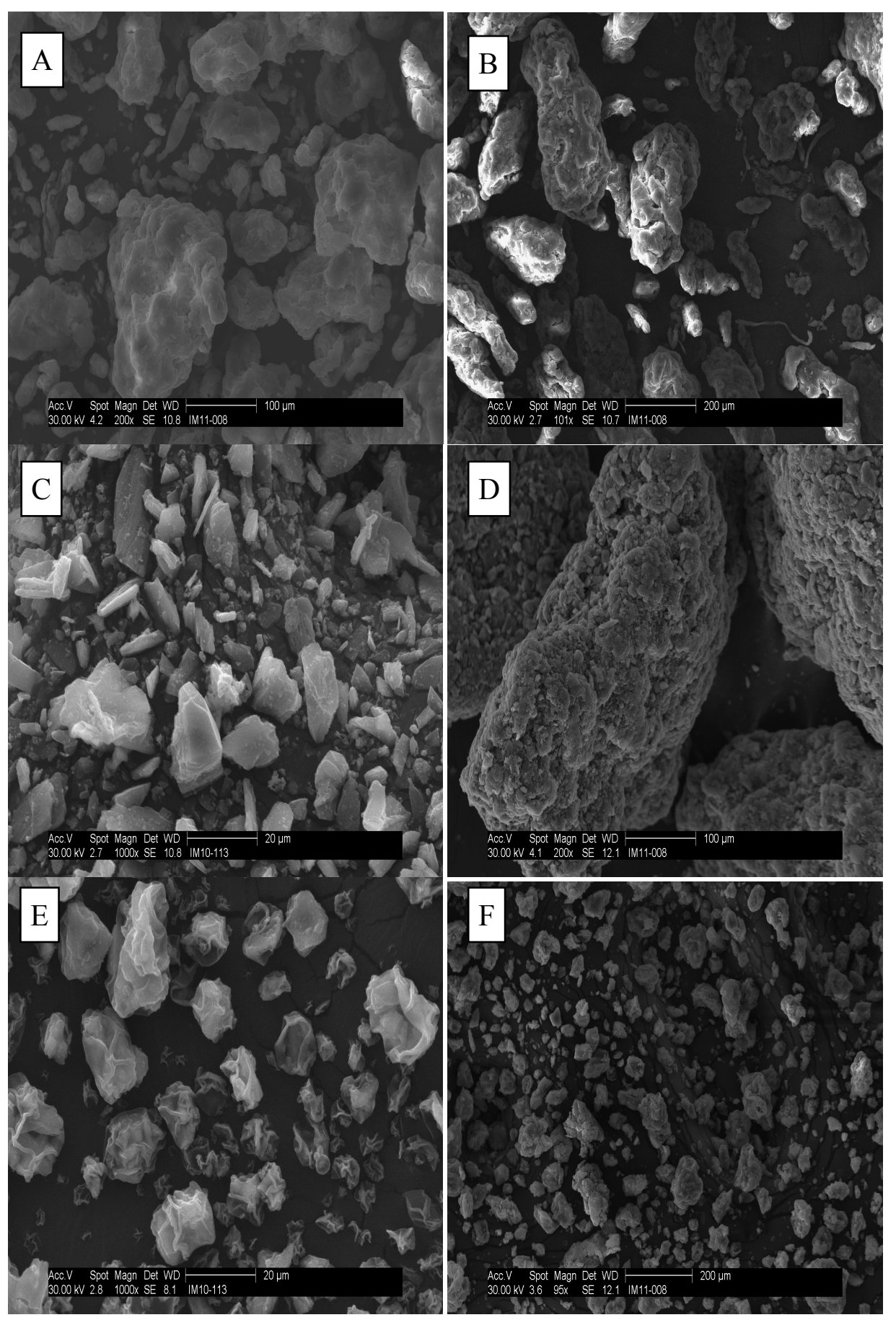

Figure 2-1. SEM image of the excipient

A: Original cellulose acetate 398-10 NF; B: CA mixed with ATBC at 2:1 ratio and heat treated for 12hours; C: milled Dicalcium phosphate; D: co-processed F2 granules by using wet granulation method; E: spray dried powder of F2 formulation; F: dry granules of spray dried F2. 
granulation process, the starting materials are milled DCP and CA mixture, the milled DCP exhibit a long prism crystalline structure under the SEM. These crystalline substances agglomerated with the CA particles after the wet granulation. The crystalline structure remains the same at the final granules as shown in Figure 2-1D. The original CA particle in the agglomerate is hard to identify because of the interaction with wet agent which contains the plasticizer during wet granulation. The CA particles dissolved or partially dissolved in the wetting agent and formed the liquid bridges which remain between the solid particles. This liquid bridge will be solidified once particles are dried. The original shape and surface of CA particles were changed and a new shape and surface were generated. Figure 2-1E showed the spray dried mixture of CA, milled DCP and plasticizer. In this mixture, CA was presented as a film form. These films remained on the surface of the milled DCP or in an individual small piece. The plasticizer completely interacted with CA and remained inside the CA film. The crystalline structure of DCP was totally covered by the plasticized CA film. The particle size of the spray dried powder was increased after dry granulation, but the film form of plasticized CA did not change in the agglomerate as shown in Figure 2-1E.

\subsubsection{The physical characteristics}

The physical properties of the co-processed excipients are influenced by the preparation methods as shown in Table 2-4. For the excipients prepared by the heat treatment method, the three formulations exhibit similar bulk density, tap density and the mean particle size. The only physical difference was observed from the powder porosity. The two formulations with plasticizer showed lower porosity than the formulation without plasticizer. This is because the plasticizer only remained on the polymer surface and did not change the size and surface of the polymer as illustrated in Figure 2-1B. The porosity decrease is due to the liquid plasticizer which filled the pores of the polymer powder. For the excipients prepared by wet granulation method, addition of the plasticizer decreases the bulk density, taps density and increases the powder porosity. These differences can be explained by two factors. Firstly, the interaction of the plasticizer and polymer results in a stronger and smoother film, therefore less particle interlock appears in the processed excipient. Secondly, the density of liquid plasticizer is less than the solid DCP. This contributes to a lower bulk density and tap density. The excipients prepared by the spray dried methods showed similar physical properties. This process method is very powerful and totally changed the physical properties of the original polymer. The resulting new physical form was not affected by the addition of the plasticizer and the type of the plasticizer. Among the three preparation methods, the heat treatment method and wet granulation method generate more density and less porous excipients and the spray dried method generates light and porous excipients. The particle size of the excipients prepared by spray dried method and wet granulation method are larger than the heat treatment method. This is because the agglomerates formed in those two processes. 
Table 2-4. Physical properties for the co-processed excipient prepared by three different methods

\begin{tabular}{lccccc}
\hline Formulations & $\begin{array}{c}\text { Bulk } \\
\text { density } \\
(\mathbf{g} / \mathbf{m l})\end{array}$ & $\begin{array}{c}\text { Tap } \\
\text { density } \\
(\mathbf{g} / \mathbf{m l})\end{array}$ & $\begin{array}{c}\text { Carr's } \\
\text { index } \\
\mathbf{( \% )}\end{array}$ & $\begin{array}{c}\text { Powder } \\
\text { porosity } \\
\mathbf{( \% )}\end{array}$ & $\begin{array}{c}\text { Geometric } \\
\text { mean } \\
\text { particle } \\
\text { size }(\boldsymbol{\mu m})\end{array}$ \\
\hline F0 (Heat treatment) & 0.67 & 0.79 & 15.75 & 69.16 & 196.34 \\
F1 (Heat treatment) & 0.66 & 0.80 & 14.78 & 65.93 & 204.51 \\
F2(Heat treatment) & 0.67 & 0.82 & 17.74 & 65.04 & 205.85 \\
F0(Wet granulation) & 0.77 & 0.88 & 18.61 & 63.50 & 299.82 \\
F1(Wet granulation) & 0.68 & 0.79 & 12.88 & 67.11 & 277.54 \\
F2(Wet granulation) & 0.63 & 0.77 & 13.54 & 68.46 & 287.71 \\
F0(Spray dried) & 0.52 & 0.68 & 21.11 & 73.88 & 249.36 \\
F1(Spray dried) & 0.55 & 0.73 & 23.42 & 73.40 & 261.44 \\
F2(Spray dried) & 0.54 & 0.69 & 22.00 & 72.60 & 247.16 \\
\hline
\end{tabular}




\subsubsection{Flow characteristics}

The flowability of the co-processed excipients is listed in Table 2-5. All the coprocessed excipients prepared in this study showed good flowability, the flowability composite index all greater than 70 . The flowability results suggested that the flowability of the co-processed excipients was only affected by the preparation method and that the formulation factors including addition of plasticizer and the type of plasticizer did not affect the powder flowability. The excipients prepared by heat treatment method and wet granulation method showed better flowability than the spray dried method. The large amount of fine powders existing in spray dried excipients as shown in Figure 2-1E is responsible for the smaller flowability index.

\subsubsection{Compactability}

\subsection{Compression profile}

The compression profile of all the co-processed excipients was shown in

Figure 2-2. The co-processed excipients prepared by spray dried method exhibit the best compactability and form the strongest tablets. Followed by the wet granulation method, poor mechanical strength was observed from the tablets prepared from the heat treatment co-processed excipients. Even at highest compression pressure, the tensile strength of both formulation F1 and F2 are smaller than 1.0. This poor mechanical strength may result in tablet breakage during rigorous handling and transportation. In all the preparation methods, addition of the plasticizer decreased the tensile strength of the tablets due to the soften effect of the plasticizer on the polymer. The type ATEC produces stronger tablets than the hydrophobic plasticizer ATBC for all three preparation methods. The compactability difference can be explained by the surface area results (Table 2-6). For plastic deformation material CA, it will only undergo plastic deformation and won't generate new surface during compression. The tensile strength of final tablet is directly related to the surface area of the material. The bigger surface area of the material, the more chance for the particles connect to each other to form solid bonding and yield stronger tablet. In our study, we found the linear relationship between the surface area and the tensile strength of the tablets at the compression force of $1.5 \mathrm{~m}$ ton. $\left(\mathrm{R}^{2}=0.94\right)$ Typically, plasticizer can penetrate to the polymer structure and make the polymer more flexible and have better mobility. Therefore, the smoother surface will be expected when we mix plasticizer with the polymer. The smooth surface has less surface area compare to the irregular surface. If the plasticizer doesn't interact very well with the polymer, only one part of the plasticizer molecules enters the polymer structure and other part remains outside of the polymer structure, the surface area of the polymer will even less because the excess liquid filled all the void space of the surface. The surface area results indicate the plasticizer ATEC has a better interaction with CA than ATBC. This result was consistence with the result of glass transition temperature. In this study, the co-processed excipient prepared by spray dried method exhibit high powder porosity and large surface area. Without the plasticizer, the wet granulated excipient has similar surface area to the 
Table 2-5. Flow properties for the co-processed excipient prepared by three different methods

\begin{tabular}{lcccc}
\hline Formulations & $\begin{array}{c}\text { Carr's } \\
\text { index } \\
(\mathbf{\%})\end{array}$ & $\begin{array}{c}\text { Critical } \\
\text { orifice } \\
(\mathbf{m m})\end{array}$ & $\begin{array}{c}\text { Angle of } \\
\text { repose } \\
\text { (degree) }\end{array}$ & $\begin{array}{c}\text { Flowability } \\
\text { composite } \\
\text { Index }\end{array}$ \\
\hline F0 (Heat treatment) & 15.75 & 5 & 26.77 & 90.54 \\
F1 (Heat treatment) & 14.78 & 5 & 26.48 & 91.38 \\
F2(Heat treatment) & 17.74 & 5 & 27.56 & 88.69 \\
F0(Wet granulation) & 18.61 & 4 & 26.48 & 89.94 \\
F1(Wet granulation) & 13.54 & 4 & 26.01 & 93.63 \\
F2(Wet granulation) & 12.88 & 4 & 26.19 & 93.95 \\
F0(Spray dried) & 21.11 & 12 & 26.00 & 79.70 \\
F1(Spray dried) & 23.42 & 12 & 27.70 & 77.04 \\
F2(Spray dried) & 22.00 & 12 & 27.60 & 78.05 \\
\hline
\end{tabular}




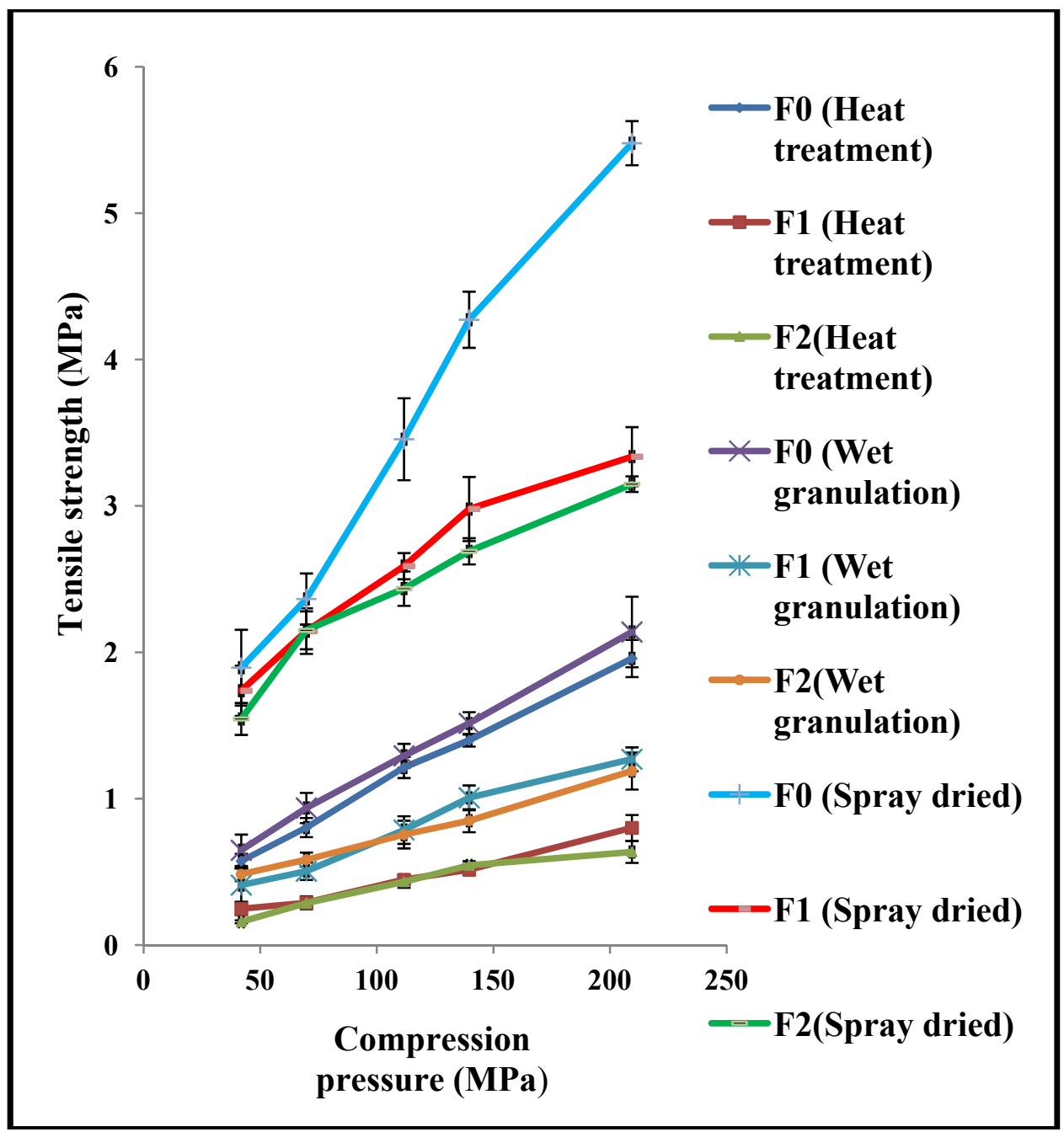

Figure 2-2. Compression profile of the co-processed excipients prepared by three different methods 
Table 2-6. Specific surface area of the co-processed excipients prepared by three different methods

\begin{tabular}{lc}
\hline Formulations & Specific surface area $\left(\mathbf{m}^{2} / \mathbf{g}\right)$ \\
\hline F0 (Heat treatment) & $2.0001 \pm 0.053$ \\
F1 (Heat treatment) & $0.2831 \pm 0.015$ \\
F2 (Heat treatment) & $0.1618 \pm 0.044$ \\
F0 (Wet granulation) & $2.3176 \pm 0.032$ \\
F1(Wet granulation) & $0.8545 \pm 0.009$ \\
F2(Wet granulation) & $0.5860 \pm 0.008$ \\
F0(Spray dried) & $7.7629 \pm 0.005$ \\
F1(Spray dried) & $3.5017 \pm 0.064$ \\
F2(Spray dried) & $2.7999 \pm 0.042$ \\
\hline
\end{tabular}


heat treated excipient. After addition of the plasticizer, the heat treated excipient showed less surface area than the wet granulated excipient for both formulations F1 and F2. This indicated the better interaction between the polymer and the plasticizer in wet granulation process.

\subsection{Heckel's plot}

Heckel's plot results (Figure 2-3) show that addition of plasticizer decreases the yield strength of the co-processed excipients for all preparation methods and the type of the plasticizer did not significantly affect the yield strength. This result suggests the plasticized excipients can deform easier than unplasticized excipient under compression force. All of the co-processed excipients formulations contain $70-80 \%$ DCP. DCP is a typical brittle material and will undergo fragmental deformation during compression. It doesn't have the plastic properties and the slope of Heckel's plot is close to zero. When DCP mixed with the plastic material CA, the mixture showed certain plastic properties, but the slope is still very small and requires more energy for deformation. A plasticizer will add plasticity to the powder mixture and less energy is required for the plasticized mixture. In our study, we also found that preparation methods affect the yield strength. The order for the yield strength of the co-processed excipients is: spray dried $<$ wet granulation $<$ heat treatment. The possible explanation could be: a) the interaction between the plasticizer and CA: the spray dried method provides the best interaction and the least interaction during the heat treatment method; b) the surface area: the larger surface exposed to the compression force, the more area available for deformation and easier plastic deformation can be expected.

\subsubsection{Effect of formulation factors}

\subsubsection{Physical characteristic}

The physical properties of the co-processed excipients prepared from different plasticizers and different concentrations of plasticizer were listed in Tables 2-7 and 2-8. All the formulations listed in Tables 2-7 and 2-8 exhibits very similar physical properties. This indicates the process method played a major role in controlling the physical properties of the co-processed excipients. The formulation factors have less influence on the physical properties of the final excipients.

\subsubsection{Flow characteristic}

Similar results were observed for flow characteristic as shown in Tables 2-9 and 2-10. All of the formulations prepared by the spray dried method showed similar flow properties. The flow characteristic of the granules is directly related to the shape and size of the granules. Because all of the formulations showed the similar density, particle size 


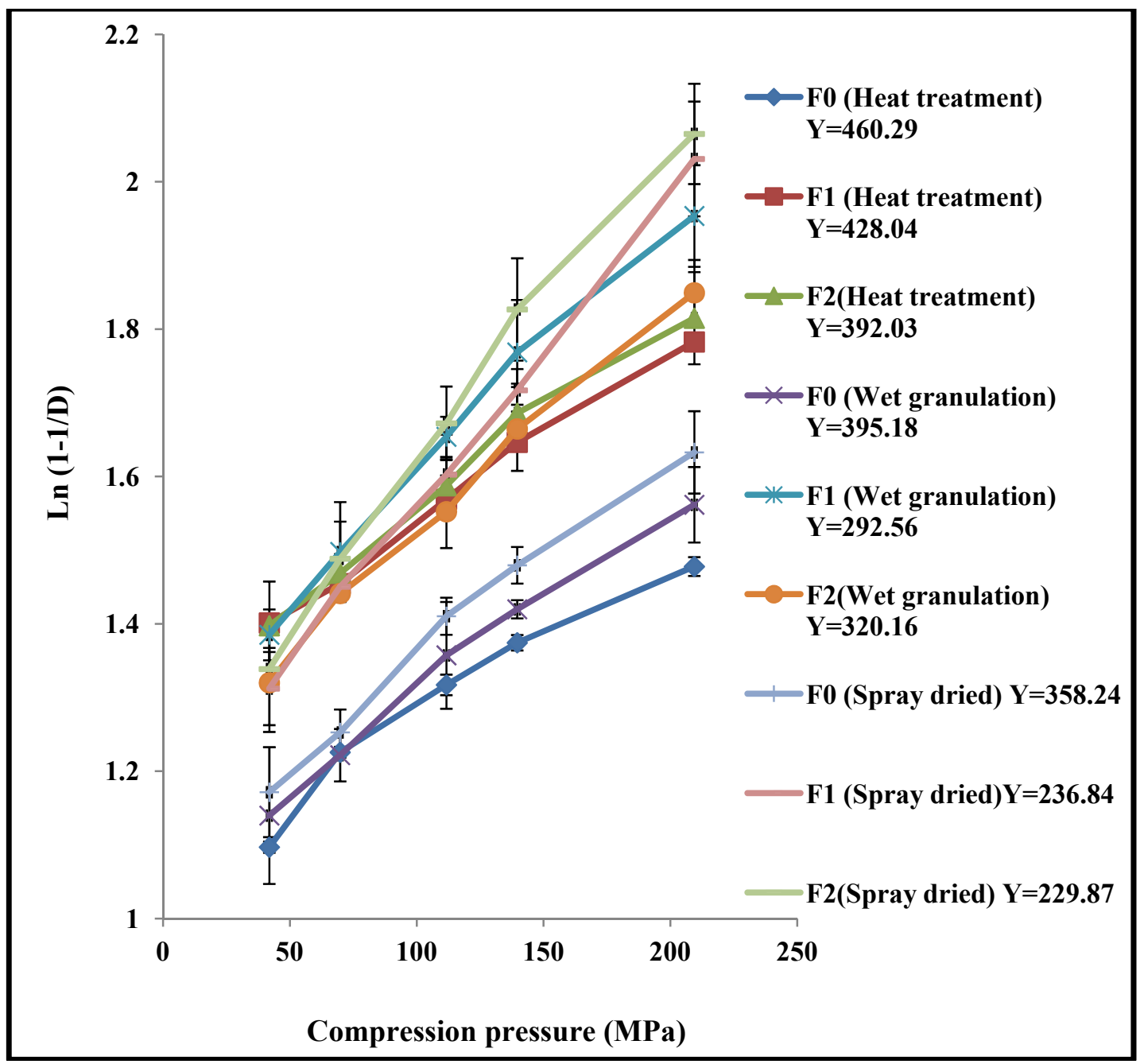

Figure 2-3. Heckel's plot of the co-processed excipients prepared by three different methods 
Table 2-7. Physical properties for the co-processed excipients prepared from different plasticizers by using spray dried method

\begin{tabular}{lccccc}
\hline Formulations & $\begin{array}{c}\text { Bulk } \\
\text { density } \\
(\mathbf{g} / \mathbf{m l})\end{array}$ & $\begin{array}{c}\text { Tap } \\
\text { density } \\
\mathbf{( g / m l )}\end{array}$ & $\begin{array}{c}\text { Carr's } \\
\text { index } \\
\mathbf{( \% )}\end{array}$ & $\begin{array}{c}\text { Powder } \\
\text { porosity } \\
\mathbf{( \% )}\end{array}$ & $\begin{array}{c}\text { Geometric } \\
\text { mean } \\
\text { particle } \\
\text { size }(\boldsymbol{\mu m})\end{array}$ \\
\hline F0 (no plasticizer) & 0.52 & 0.68 & 21.11 & 73.88 & 249.36 \\
F1(ATEC) & 0.55 & 0.73 & 23.42 & 73.40 & 261.44 \\
F2(ATBC) & 0.54 & 0.69 & 22.00 & 72.60 & 247.16 \\
F3(TEC) & 0.54 & 0.67 & 18.35 & 71.32 & 267.94 \\
F4(DBS) & 0.55 & 0.73 & 25.44 & 71.39 & 278.92 \\
\hline
\end{tabular}

Table 2-8. Physical properties for the co-processed excipients prepared from different concentrations of plasticizer by using spray dried method

\begin{tabular}{lccccc}
\hline Formulations & $\begin{array}{c}\text { Bulk } \\
\text { density } \\
(\mathbf{g} / \mathbf{m l})\end{array}$ & $\begin{array}{c}\text { Tap } \\
\text { density } \\
\mathbf{( g / m l )}\end{array}$ & $\begin{array}{c}\text { Carr's } \\
\text { index } \\
\mathbf{( \% )}\end{array}$ & $\begin{array}{c}\text { Powder } \\
\text { porosity } \\
\mathbf{( \% )}\end{array}$ & $\begin{array}{c}\text { Geometric } \\
\text { mean particle } \\
\text { size }(\boldsymbol{\mu m})\end{array}$ \\
\hline F0(no plasticizer) & 0.52 & 0.68 & 21.11 & 73.88 & 249.36 \\
F5(2.5\%ATBC) & 0.52 & 0.67 & 22.16 & 74.07 & 257.36 \\
F6(5\% ATBC) & 0.53 & 0.66 & 18.97 & 73.35 & 247.50 \\
F2(10\%ATBC) & 0.54 & 0.69 & 22.00 & 72.60 & 247.16 \\
\hline
\end{tabular}


Table 2-9. Flow properties for the co-processed excipient prepared by different plasticizers by using spray dried method

\begin{tabular}{lcccc}
\hline Formulations & $\begin{array}{c}\text { Carr's } \\
\text { index (\%) }\end{array}$ & $\begin{array}{c}\text { Critical } \\
\text { orifice(mm) }\end{array}$ & $\begin{array}{c}\text { Angle of repose } \\
\text { (degree) }\end{array}$ & $\begin{array}{c}\text { Flowability } \\
\text { composite } \\
\text { index }\end{array}$ \\
\hline F0(no plasticizer) & 21.11 & 12 & 26.00 & 79.70 \\
F5(2.5\%ATBC) & 22.16 & 12 & 27.76 & 77.83 \\
F6(5\% ATBC) & 18.97 & 12 & 27.07 & 80.42 \\
F2(10\%ATBC) & 22.00 & 12 & 27.60 & 78.05 \\
\hline
\end{tabular}

Table 2-10. Flow properties for the co-processed excipient prepared by different concentrations of plasticizer by using spray dried method

\begin{tabular}{lcccc}
\hline Formulations & $\begin{array}{c}\text { Carr's } \\
\text { index (\%) }\end{array}$ & $\begin{array}{c}\text { Critical } \\
\text { orifice(mm) }\end{array}$ & $\begin{array}{c}\text { Angle of repose } \\
\text { (degree) }\end{array}$ & $\begin{array}{c}\text { Flowability } \\
\text { composite } \\
\text { index }\end{array}$ \\
\hline F0 (no plasticizer) & 21.11 & 12 & 26.00 & 79.70 \\
F1 (ATEC) & 23.42 & 12 & 27.70 & 77.04 \\
F2 (ATBC) & 22.00 & 12 & 27.60 & 78.05 \\
F3 (TEC) & 18.35 & 14 & 30.48 & 76.34 \\
F4 (DBS) & 25.44 & 14 & 27.36 & 73.69 \\
\hline
\end{tabular}


distribution and particle shape, the similar flow properties can be expected. The flowability index for the formulations is greater than 70, which suggest the good flowability of the granules and suitable for direct compression.

\subsubsection{Compactability}

\subsection{Compression profile}

The compression profile of the co-processed excipients was affected by the type of plasticizers and the concentrations of plasticizer as shown in Figures 2-4 and 2-5. The tensile strength was decreased after addition of different type of plasticizers. The tablets containing the most hydrophilic plasticizer TEC showed the highest tensile strength, the lowest tensile strength was observed for tablets containing plasticizer DBS. The reasons of the tensile strength difference caused by the type of plasticizer can be explained by the hydrophobicity of the plasticizer and the interaction of plasticizer with CA. Based on the Log P value, the hydrophobicity of plasticizers used in this study is:

ATBC $>$ DBS $>$ ATEC $>$ TEC. The hydrophobic plasticizer is prone to contribute to the hydrogen bonding between the solid particles, therefore better mechanical strength. For the plasticizers with similar hydrophobicity, the interaction of the plasticizer with CA played a role. The interaction of the plasticizer with CA can be assessed by the glass transition temperature of CA. The glass transition temperature result is shown in Figure 2-6. Four plasticizers used in this study all can decrease the glass transition temperature of CA. The most effective plasticizer is ATEC which showed lowest glass transition temperature. The order of glass transition temperature of the plasticized CA is ATEC $<$ TEC $<$ ATBC $<$ DBS. For the least effective plasticizer DBS, there is little interaction with the polymer and phase separation will take place during compression. The extra liquid available between the solid particles will affect the formation of solid bonding. The significant decrease of the tensile strength was observed.

The effect of plasticizer concentration on the compression profile was demonstrated in Figure 2-5. When the concentration of plasticizer is below 5\%, addition of the plasticizer increases the tensile strength of the tablets. But when the concentration of plasticizer climbs to $10 \%$, the presence of plasticizer decreases the mechanical strength of the tablets. The similar phenomenon was observed when compressing MCC with a small amount of water as plasticizer and compressing HPMC with a small amount of water as plasticizer $[5,80]$. In their study [80], it was found that bonding area increased after adding the plasticizer. But upon increasing the plasticizer concentration, the bonding strength is much reduced due to the softening effect of the plasticizer on the polymer. This effect is stronger than the effect of increasing the bonding area. This explained the effect of plasticizer concentration on the tensile strength of tablets. 


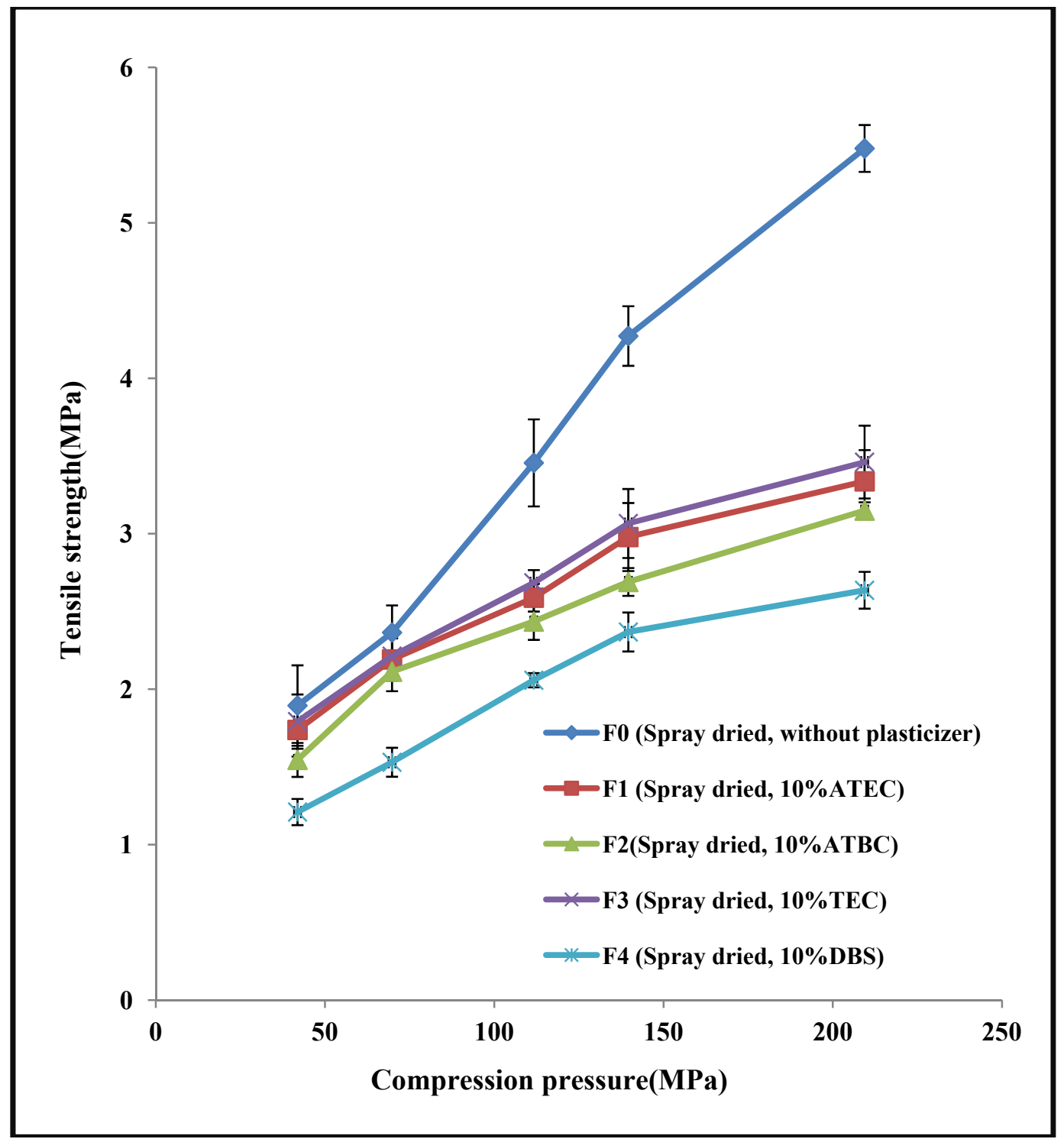

Figure 2-4. Compression profile of the co-processed excipients prepared from different plasticizers by using spray dried method 


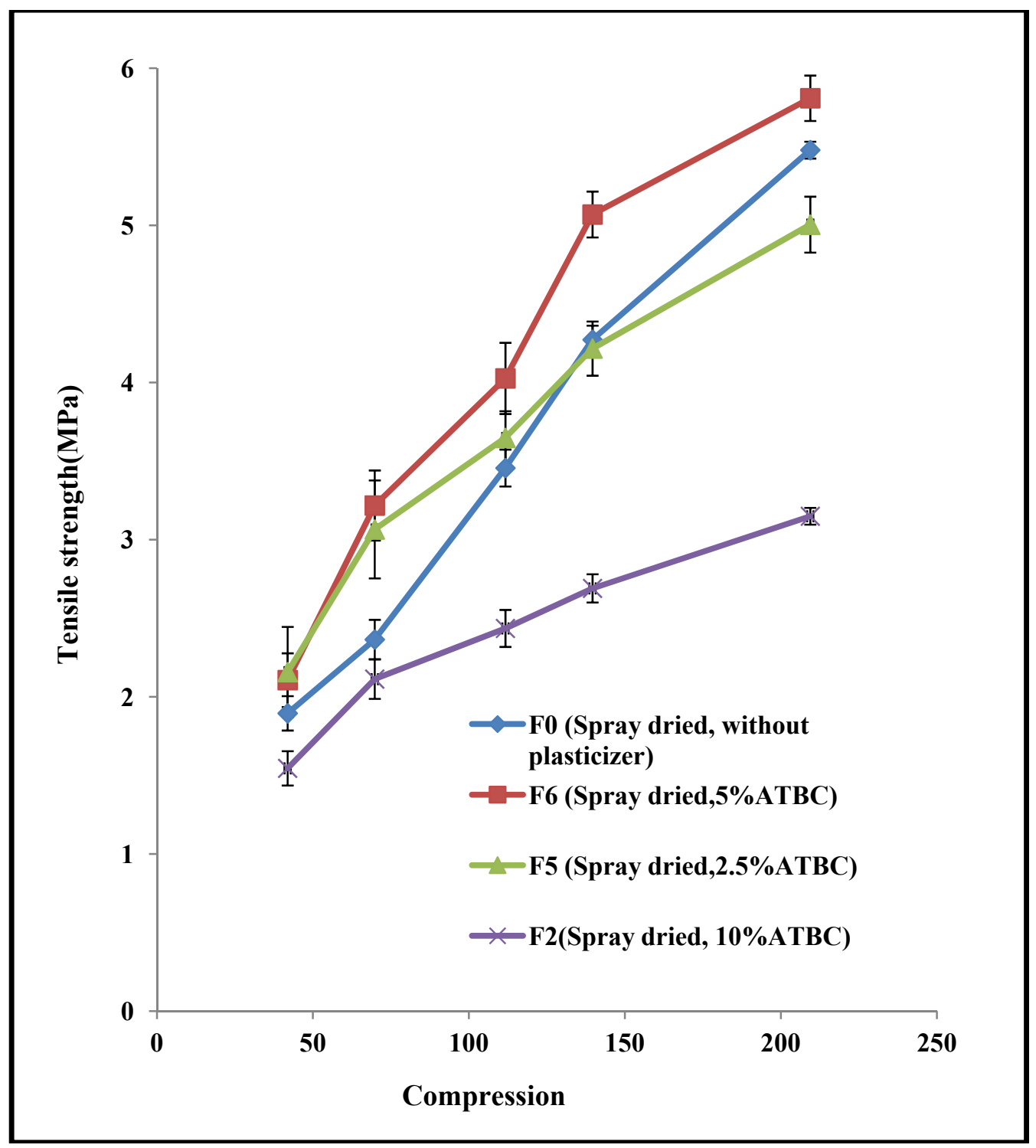

Figure 2-5. Compression profile of the co-processed excipients prepared from different concentrations of plasticizer by using spray dried method 


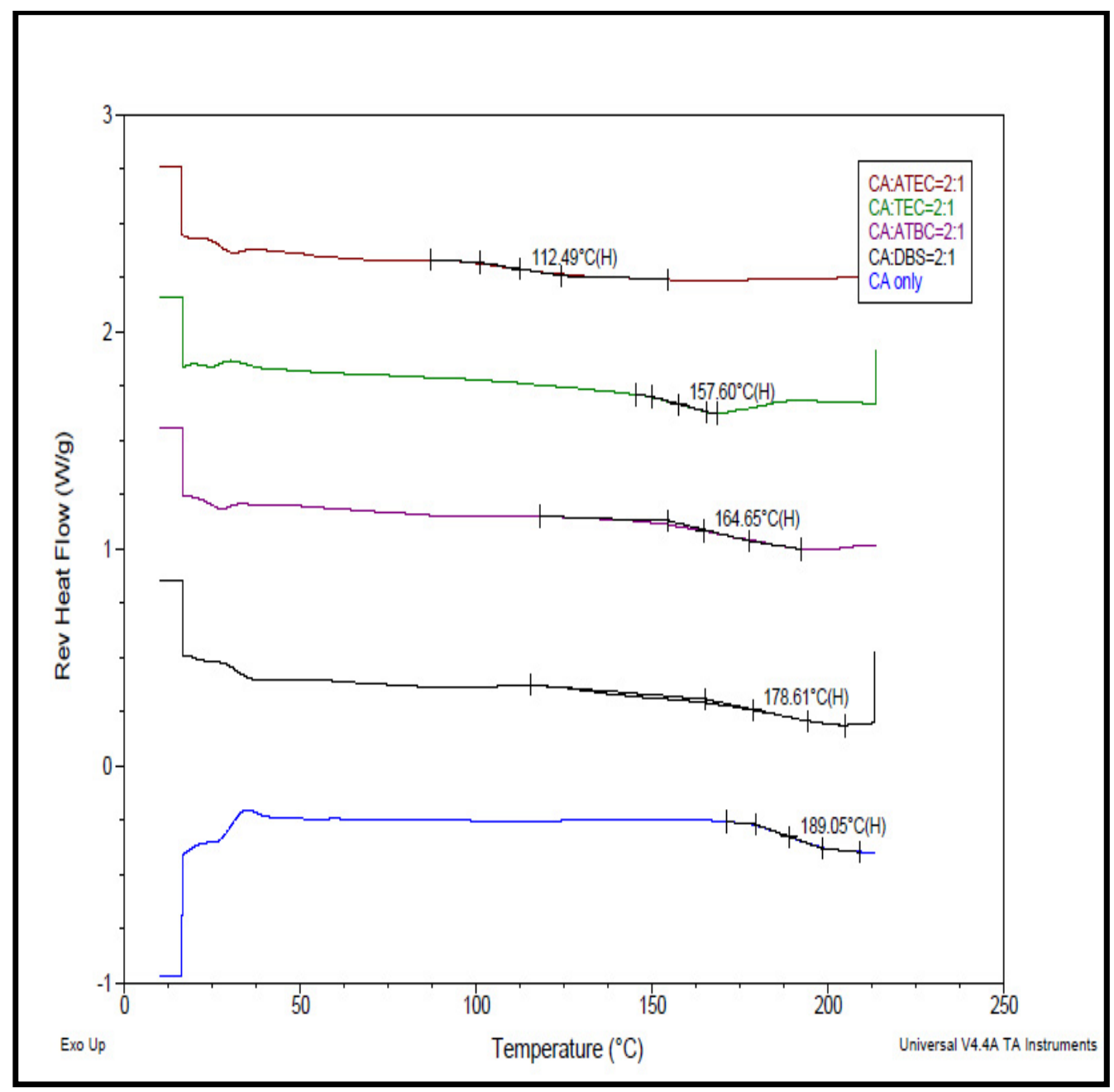

Figure 2-6. Glass transition temperature of CA with different plasticizers 


\subsection{Heckel's plot}

The effect of plasticizers on Heckel's plot is demonstrated in Figure 2-7. All the plasticizers used in this study lower the yield strength. The type of plasticizer did not affect the yield strength of co-processed excipients, but the concentration of plasticizer affects the plasticity of the co-processed excipients. As shown in Figure 2-8, the yield strength of the co-processed excipient is decreased when increasing the plasticizer concentration. This suggests increased plasticity of the co-processed excipient with high concentration of plasticizer.

\subsection{Conclusions}

The co-processed excipients with CA, plasticizer and inert excipient were successfully prepared by using three different methods. The physical, flow and compaction properties of the co-processed excipients were influenced by the formulation factors as well as the process methods. We found the physical and flow properties are controlled by the process methods and the formulation factors have less impact.

The co-processed excipients prepared in this study all showed good flow characteristic. The flowability of heat treated and wet granulated co-processed excipients are better than the spray dried excipients. The compactability of the co-processed excipients is affected by both process methods and formulation factors. The spray dried process produced more porous particles with larger surface area. These particles required the least energy for plastic deformation during compression and formed the tablets with highest mechanical strength. The wet granulated excipients showed moderate plastic deformation capacity and resulting tablets with acceptable tensile strength. The heat treated excipients required more energy to deform under compression pressure than the other two methods. The resulting tablets have poor mechanical property.

For all preparation methods, addition of $10 \%$ plasticizer increases the plasticity of the final excipient and decreases the tablet tensile strength. The tablets containing hydrophilic plasticizer showed better tensile strength than the hydrophobic plasticizer. The increased interaction between the plasticizer and the polymer also results in tablets with better tensile strength. The concentration of plasticizer also affects the compactability of the co-processed excipient. The tensile strength of the tablets increased at low plasticizer concentration and the reverse effect was observed on higher plasticizer concentration. Based on results obtained from the study, both wet granulated and spray dried excipients showed desired flowability and acceptable compactability. All of them appear to be satisfactory excipient candidates for direct compression formulation, but the controlled release capacity of these excipients needs to be investigated. 


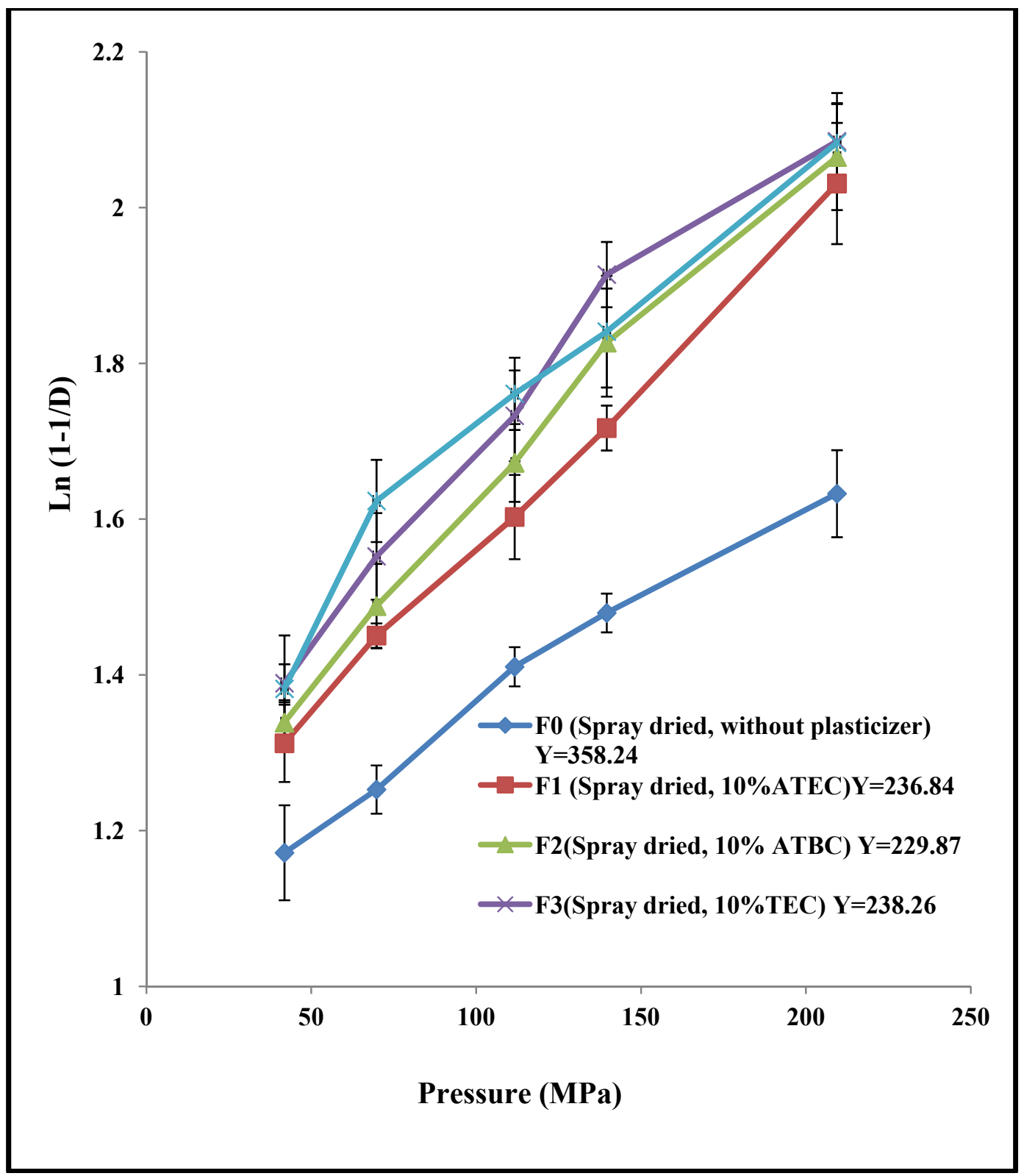

Figure 2-7. Heckel's plot of the co-processed excipients prepared from different plasticizers by using spray dried method 


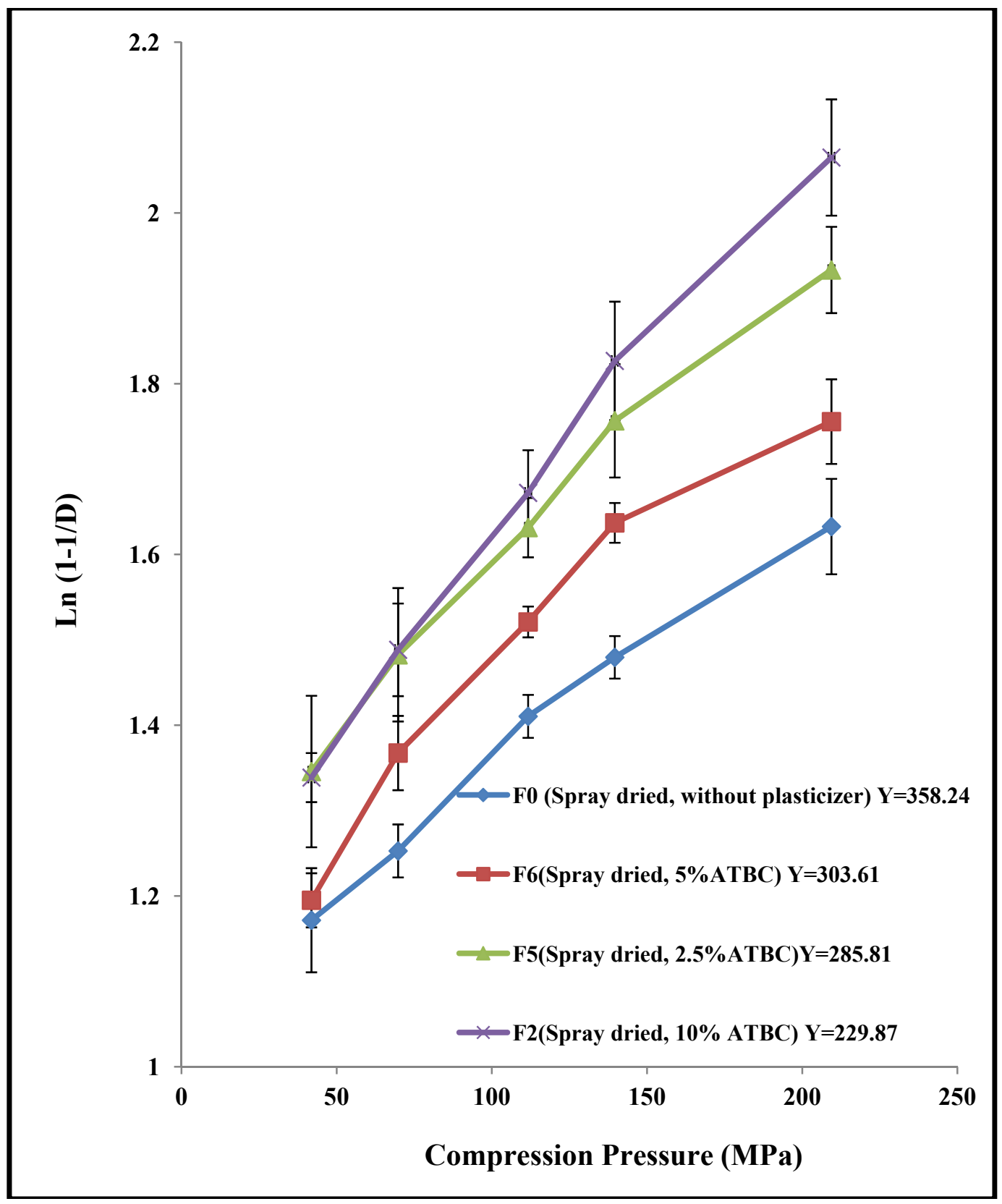

Figure 2-8. Heckel's plot of the co-processed excipients prepared from different concentrations of plasticizer by using spray dried method 


\section{Chapter 3. Sustained Release Properties and Drug Release Mechanism Study of a Novel Co-processed Excipient}

\subsection{Introduction}

The co-processed plasticized cellulose acetate with inert excipients was successfully developed by three different methods as described in chapter two. Those coprocessed excipients showed good flowability, acceptable compactability and suitability for direct compression.

In this chapter, we investigated the sustained release properties of the novel coprocessed excipients and the factors which can affect the drug release from the matrix tablets. The drug release mechanism for the matrix tablets was also studied by using two empirical mathematic equations. A freely water soluble drug propranolol hydrochloride was selected as the model drug to study the drug release characteristics from the matrices. The sparingly water soluble drug theophylline and water insoluble drug flurbiprofen were used to evaluate the drug solubility effect on the dissolution profile.

\subsection{Materials and Methods}

\subsubsection{Materials}

Cellulose acetate with $39.8 \%$ acetyl content and $38 \mathrm{cp}$ viscosities (CA 398-10 NF, Eastman, Kingsport, TN) was used as the main polymer in this study. Triethyl citrate (TEC), acetyltriethyl citrate (ATEC), acetyltributyl citrate (ATBC) and dibutyl sebacate (DBS) (Morflex Inc., Greensboro, NC) were used as plasticizers. Regular dicalcium phosphate dihydrate (DCP, Rhodia Inc., Blue Island, IL) and milled dicalcium phosphate dihydrate (milled DCP Innophos Inc., Cranbury, IL) were used as filler. Magnesium stearate (Mallinckrodt, Paris, KY) was used as a lubricant. Propranolol hydrochloride was used as water soluble model drug, Theophylline anhydrous (BASF, Ludwigshafen, Germany) was used as sparingly water soluble drug and flurbiprofen (Sun Pharma, Maharashtra, India) was used as a water insoluble drug.

\subsubsection{Methods}

\subsubsection{Tablets preparation}

For comparison purpose, the tablets containing different concentrations of unprocessed CA were prepared by a direct compression method. The formulations are listed in Table 3-1. Tablets were compressed on a Carver Press (Carver Inc., Wabash, IN) by using $3 / 8$ inch flat face punch at 1.5 metric ton compression force. The dwell time 
Table 3-1. Formulations of the tablets prepared from the unprocessed CA

\begin{tabular}{lccc}
\hline \multirow{2}{*}{ Ingredients } & \multicolumn{3}{c}{ Percentage (\%) } \\
\cline { 2 - 4 } & $\mathbf{A 1}$ & $\mathbf{A 2}$ & $\mathbf{A 3}$ \\
\hline Propranolol hydrochloride & 20 & 20 & 20 \\
CA 398-10 NF & 15 & 30 & 50 \\
Regular DCP & 64.5 & 49.5 & 29.5 \\
Magnesium stearate & 0.5 & 0.5 & 0.5 \\
Total & 100 & 100 & 100 \\
\hline
\end{tabular}


is 3 seconds and the target weight is $400 \mathrm{mg}$.

Tablets containing the co-processed excipient were also prepared by direct compression method. All the tablets prepared by the co-processed excipients share the same formula. All the tablets contain $20 \%$ drug, $79.5 \%$ co-processed excipient and $0.5 \%$ magnesium stearate. The ingredients were mixed by passing through a 20 mesh sieve 3 times and then compressed into $400 \mathrm{mg}$ tablets at the same compression condition as the unprocessed $\mathrm{CA}$ tablets. In order to investigate the compression force effect, two additional compression forces $0.5 \mathrm{~m}$ ton and $1.0 \mathrm{~m}$ ton also applied.

\subsubsection{In vitro dissolution study}

The USP Apparatus II (Hanson SR-8 Plus, Hanson Research Corporation, Chatsworth, CA) was used to evaluate the drug release. Three tablets from each batch were tested. $900 \mathrm{ml}$ of distilled water, $\mathrm{pH} 7.4$ phosphate buffer and $\mathrm{pH} 7.2$ phosphate buffer at $37 \pm 0.5^{\circ} \mathrm{C}$ was used as the dissolution medium for evaluate the release of propranolol hydrochloride, theophylline and flurobiprofen respectively. The samples were taken at predetermined time points for 12 hours. The absorbance of the solution was measured at $289 \mathrm{~nm}, 274 \mathrm{~nm}$ and $277 \mathrm{~nm}$ respectively by a UV-spectrophotometer (Perkin-Elmer Corporation, Norwalk, CT). The drug concentration and the percentage of drug release were calculated by the software DissLab ${ }^{\circledR}$.

In order to determine the $\mathrm{pH}$ effect on dissolution profile, $900 \mathrm{ml}$ simulated gastric fluid (without enzymes) and simulated intestine fluid (without enzymes) were used as dissolution medium. The tablets prepared by mixing propranolol hydrochloride and spray dried co-processed excipient $\mathrm{F} 2$ for evaluation. The drug dissolution profile was compared by using $f_{2}$ value as described in Equation 3-1.

$$
f_{2}=50 \times \log \left\{\left[1+(1+1 / \mathrm{n}) \sum_{\mathrm{t}=1}\left(\mathrm{R}_{\mathrm{t}}-\mathrm{T}_{\mathrm{t}}\right)^{2}\right]^{-0.5} \times 100\right\}
$$

where $f_{2}$ is a similarity factor, $n$ is the number of sample times, $R_{t}$ is the average percentage of drug released at time $t$ for the reference sample, $T_{\mathrm{t}}$ is the average percentage of drug released at time $t$ for the test sample. According to FDA's guidance for industry, if $f_{2}$ values were greater than 50 , the two dissolution profiles can be consider as similar or equivalent.

\subsubsection{Tablet porosity measurement}

The true density $\left(\rho_{t}\right)$ of the lubricated drug and co-processed excipients mixture were determined by a helium pycnometer (AccuPyc 1330, Micromeritics, Norcross, GA). The density of the tablet $\left(\rho_{\mathrm{a}}\right)$ was calculated from Equation 3-2.

$$
\rho_{\mathrm{a}}=\pi^{*} \mathrm{r}^{2 *} 1
$$


where $r$ is the radial of the tablet and $l$ is the thickness of the tablet.

The porosities of the tablets $(\varepsilon)$ were calculated from the tablet density ( $\rho$ a) and true density ( $\rho t)$ of the mixture using the following Equation 3-3:

$$
\varepsilon=100\left(1-\rho_{\mathrm{a}} / \rho_{\mathrm{t}}\right)
$$

\subsubsection{Tablet surface examination}

The surface of tablets was first coated by $10 \mathrm{~nm}$ gold particles for improving the conductivity. The morphology of the tablets surface was examined by the FEI XL-30 Scannig Electron Microscopy. (SEM, FEI, Hillsboro, OR)

\subsubsection{Initial water penetration study}

The initial water penetration study of the tablets was performed in a $0.1 \%$ methylene blue solution. Tablets was first dipped into this solution for 1 minute, then taken out, wiped the excess liquid and cut into half to observe the surface and the cross section under the Olympus MIC-D microscope. (Olympus, Center Valley, PA).

\subsubsection{Drug solubility measurement}

The excess amount of drug was put in the corresponding dissolution medium, then vortexed for 3 minutes. The mixture was shaken for 7 days at $37^{\circ} \mathrm{C}$ to reach equilibrium. The sample was taken and centrifuged at $10000 \mathrm{rpm}$ for 10 minutes. The supernatant was filtered by a $0.45 \mu \mathrm{m}$ membrane filter. The filtrate was diluted by the dissolution medium and the concentration of the dilution was determined by UVSpectrophotometer (Perkin-Elmer Corporation, Norwalk, CT).

\subsubsection{Drug release mechanism study}

Drug-release mechanism was studied by fitting the dissolution data to two empirical mathematical equations including Higuchi equation and power law.

The Higuchi equation was developed in $1961[11,36]$ to describe the drug release from the inert matrix tablets. The equation is as following:

$$
\mathrm{Q}_{\mathrm{t}}=\left[\frac{\mathrm{S}^{2} \mathrm{D} \varepsilon}{\tau}\left(2 \mathrm{C}_{0}-\varepsilon \mathrm{C}_{\mathrm{S}}\right) \mathrm{C}_{\mathrm{S}} \mathrm{t}\right]^{\frac{1}{2}}=\mathrm{k} \mathrm{t}^{\frac{1}{2}}
$$

where $\mathrm{Qt}$ is the cumulative amount of drug released over a surface unit in time $t ; \mathrm{S}$ is the surface area of the tablet; D denotes the drug diffusion coefficient in the matrix phase; Cs 
is the drug solubility in the matrix; $\mathrm{C}_{0}$ represents the drug concentration in the matrix; $\varepsilon$ is the porosity; $\tau$ denotes the tortuosity of the matrix; and $\mathrm{k}$ is the dissolution rate constant.

Based on this equation, drug release at any given time is proportional to the square root of the time. The release rate constant $\mathrm{k}$ can be calculated from the dissolution profile. $\mathrm{D} \varepsilon / \tau$ (effective diffusion coefficient) also can be calculated from the constant $\mathrm{k}$ and known drug and tablet properties.

The general equation of the power law [45] is Equation 3-5.

$$
\mathrm{M}_{\mathrm{t}} / \mathrm{M}_{\infty}=\mathrm{k}^{*} \mathrm{t}^{\mathrm{n}}
$$

where $\mathrm{M}_{t}$ is cumulative amount of drug released at time $t, \mathrm{M}_{\infty}$ is cumulative amount of drug released at infinite time; $\mathrm{k}$ is release constant incorporating structural and geometric characteristics of the system, and $\mathrm{n}$ is the release exponent indicating the mechanism of drug release.

The release exponent ' $n$ ' in the power law takes multiple values based on geometry of the system. For a cylinder shape tablets, if $\mathrm{n}<0.45$, the major release mechanism is Fickian diffusion; if the $\mathrm{n}$ value $>0.89$, Case-II transport in which the polymer relaxation is the rate limiting process; if $\mathrm{n}$ value between 0.45 and 0.89 , the "anomalous transport" takes place where both of drug diffusion and swelling of the polymer are involved.

Addition to model fit, the geometric dimension of the tablets prepared with drugs of different solubility was measured before and after the dissolution by using the caliper.

\subsection{Results and Discussion}

\subsubsection{Dissolution profile of unprocessed CA}

The previous research on CA for matrix tablet polymer demonstrated that the CA matrices are sensitive to the solubility of the drug [61]. As a retarding agent, it not suitable for controlling the release for freely water soluble drug from the matrix tablet. Our study showed in Figure 3-1 has an agreement with results from the literature. When the concentration of $\mathrm{CA}$ is $15 \%$ and $30 \%$, more than ninety percent drug released within 1 hour. Even the concentration of CA reaches to $50 \%$, eighty percent of drug released within 2.5 hours. Because of the low bulk density of CA $(0.289 \mathrm{~g} / \mathrm{ml})$, the mixtures with higher concentration of CA exceeded the volume limitation of the die. Tablet with higher concentrations of CA was not prepared in this study. These results confirmed that the unprocessed CA could not be used to formulate the sustained release matrix tablets for freely water soluble drug at concentrations less than $50 \%$. 


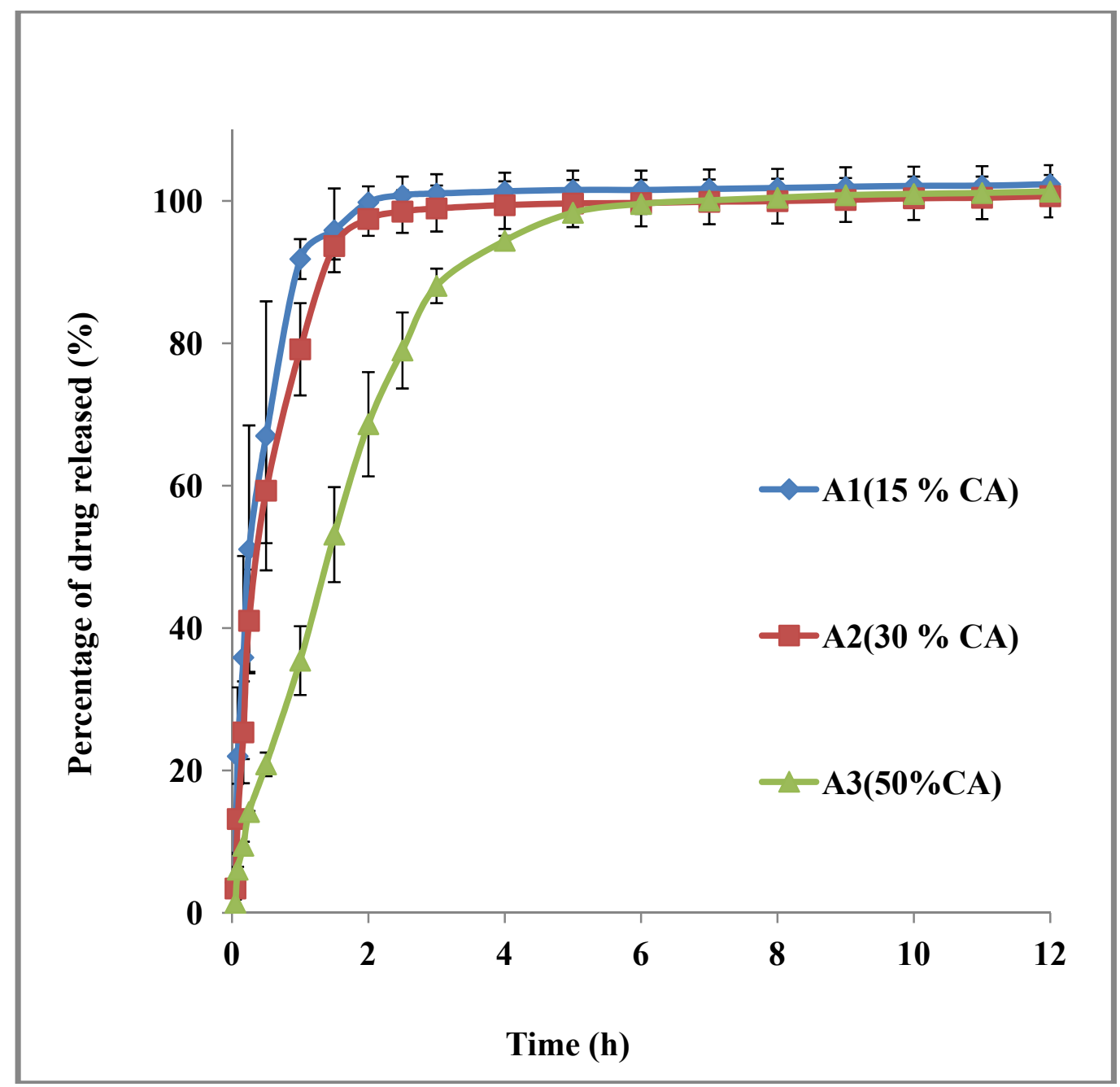

Figure 3-1. Dissolution profile of the tablets containing unprocessed CA 


\subsubsection{The effect of preparation process of co-processed excipients on drug release}

\subsubsection{Dissolution profile}

The dissolution profile (Figure 3-2) shows that drug release characteristic was directly related to the preparation process and formulation factors of the co-processed excipients. All the tablets prepared from the heat treatment co-processed excipient have a rapid dissolution rate. More than $90 \%$ drug was released in the first hour. The tablets prepared from the wet granulated co-processed excipients show slower drug release rate. The slowest drug release rate was obtained from the tablets prepared from spray dried coprocessed excipient. For the co-processed excipients prepared from wet granulation method and spray dried method, addition of the plasticizer significantly decreased the drug release rate and the hydrophobic plasticizer ATBC demonstrated slower release rate than the hydrophilic plasticizer ATEC. Since the spray dried excipients provided the slowest sustained release profile among these three methods, it was selected for further investigation. The difference in drug release profiles can be explained by tablet porosity, tablet surface morphology and initial water penetration rate as discussed below.

\subsubsection{Tablet porosity}

For a hydrophobic matrix tablet, irregular pores will be formed during compression due to the boundaries of the particles. For this system, drug release must be preceded by the drug dissolution in medium filled pores and by diffusion through medium filled channels. The size, structure and amount of the pores are very important for controlling the drug release [81].

The porosity of the tablets is listed in Table 3-2. For all the co-processed excipient, despite of the preparation method, addition of plasticizer significantly decreases the porosity of the tablets $(p<0.05)$. Fewer diffusion channels will be presented due to the decreased porosity. Therefore, the slower drug release profile was observed for the plasticized matrices than the unplasticized matrices. But the type of the plasticizer and the excipient preparation methods did not significantly affect the porosity of the tablets $(\mathrm{p}>0.05)$. Even though there is a clear dissolution difference among the tablets with different co-processed excipients, we could attribute the difference in porosity of the tablets alone. This result suggests the overall porosity is not the only factor which has influence on the drug dissolution. Other factors such as hydrophobicity, size and tortuosity are also expected to play an important role in controlling the drug release from the matrix system.

\subsubsection{Tablet surface morphology}

Figure 3-3 showed the surface morphology of the tablets prepared from coprocessed excipient formula F2 with $20 \%$ propranolol hydrochloride. The surface of the 


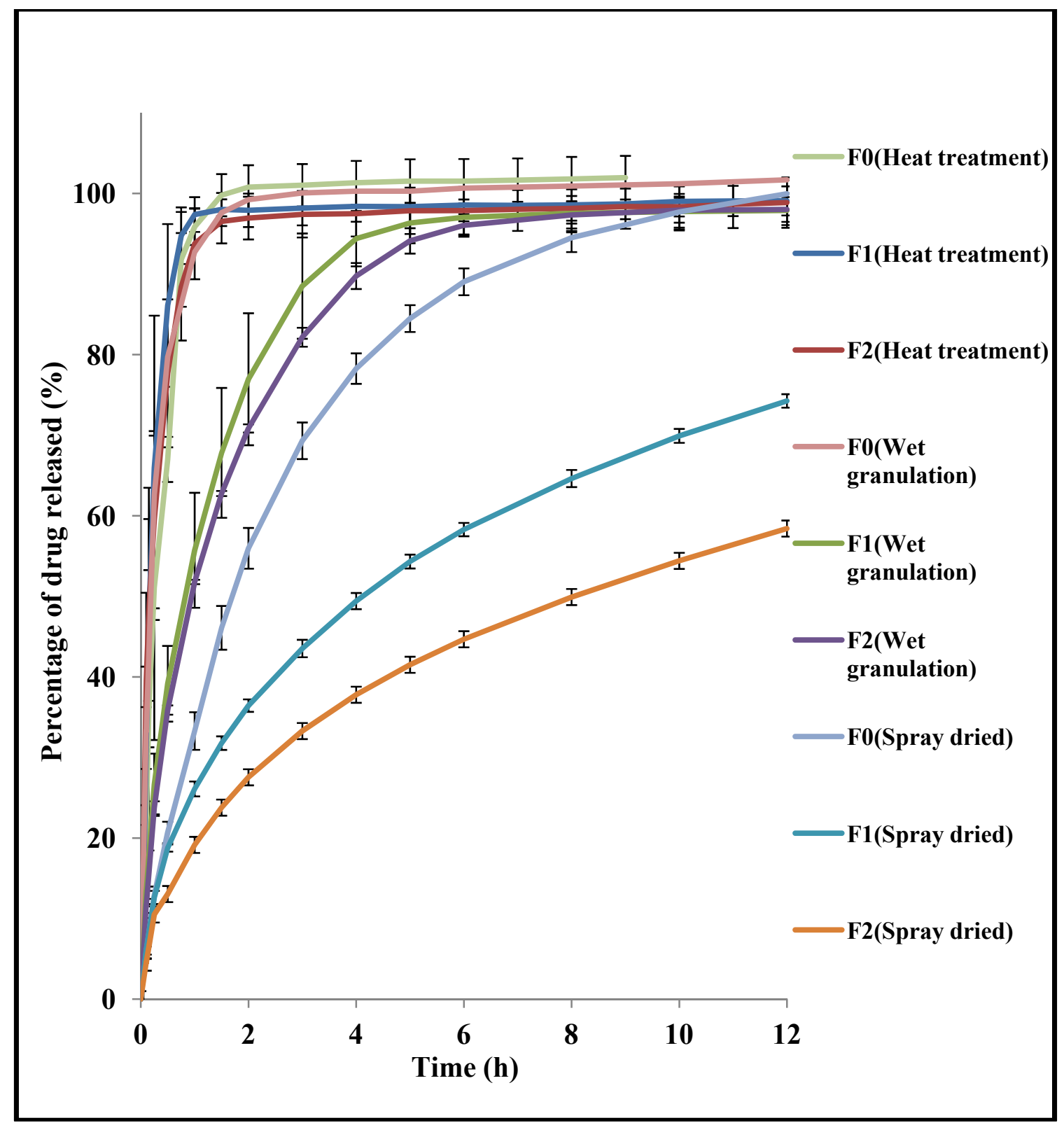

Figure 3-2. Dissolution profile of tablets containing the co-processed excipients prepared from different process methods 
Table 3-2. Porosity of the tablets prepared by propranolol hydrochloride and different co-processed excipients

\begin{tabular}{lccc}
\hline Preparation of co- & \multicolumn{3}{c}{ Porosity (\%) } \\
\cline { 2 - 4 } processed excipients & F0 & F1 & F2 \\
\hline Heat treatment & $17.06 \pm 1.00$ & $10.12 \pm 1.60$ & $10.77 \pm 0.54$ \\
Wet granulation & $17.30 \pm 1.34$ & $10.68 \pm 0.72$ & $10.92 \pm 1.25$ \\
Spray drying & $16.95 \pm 1.48$ & $9.37 \pm 0.65$ & $9.53 \pm 0.85$ \\
\hline
\end{tabular}




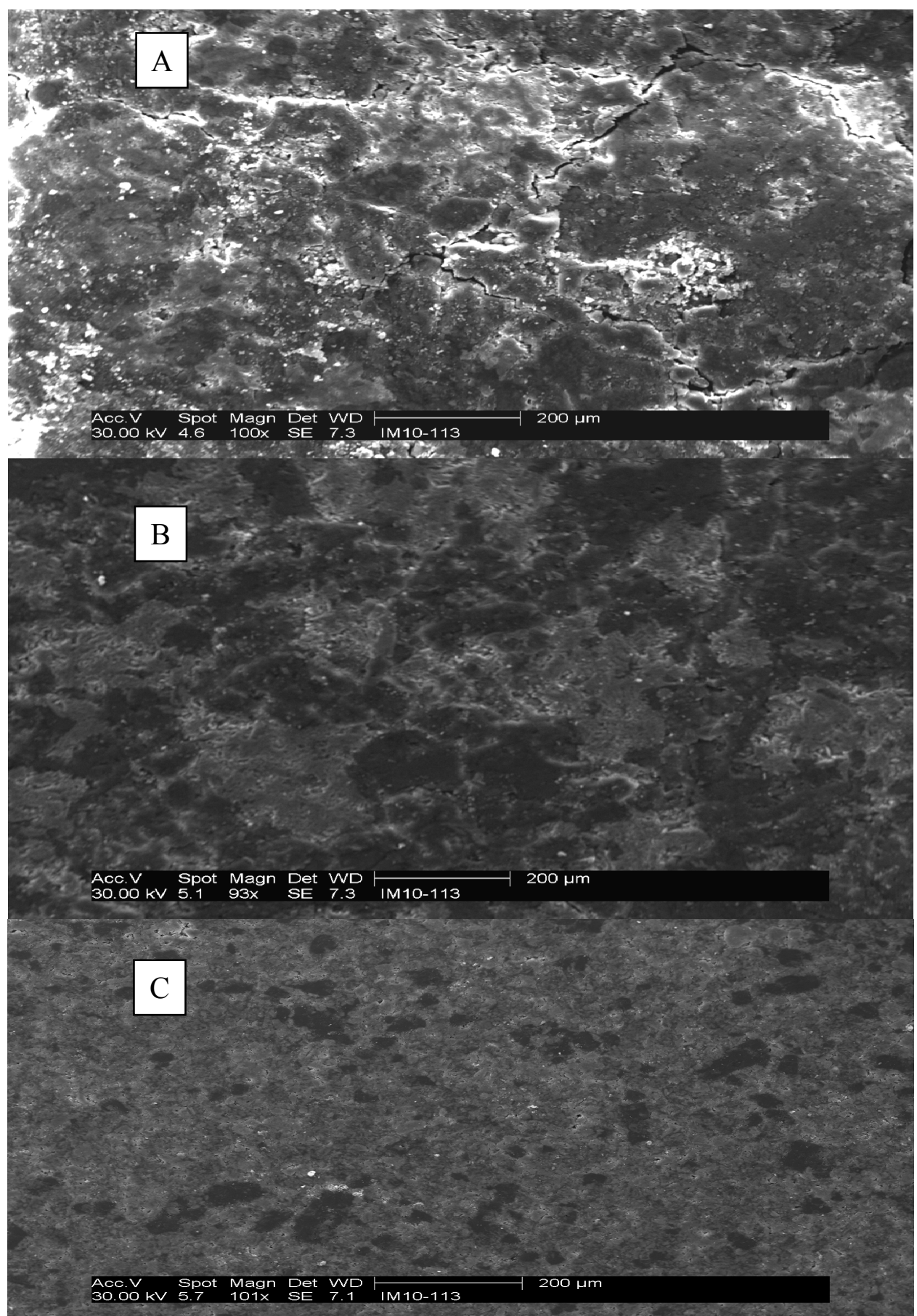

\section{Figure 3-3. SEM of tablet surface}

A: Tablet containing 20\% propranolol and 79.5\% heat treated excipient F2; B: tablet containing $20 \%$ propranolol and $79.5 \%$ wet granulated excipient F2; C: tablet containing $20 \%$ propranolol and $79.5 \%$ spray dried excipient F2. 
tablets prepared from the heat treatment excipient showed several big cracks, these cracks can act as channels for water penetration and drug diffusion, therefore resulting faster drug release. Small cracks were observed on the surface of the tablet prepared from the wet granulated excipient. A smooth and film-like surface was observed from the tablet prepared from the spray dried excipient. The small and individual pores were presented uniformly in the entire surface. This uniform structure can effectively reduce the water penetration and result in a prolonged drug release. These observations reveal the surface characteristics and the effect of pore structure on water penetration and can be used for explaining the dissolution differences among the preparation methods of co-processed excipients. This finding is in good agreement with the literature [61].

\subsubsection{Initial water penetration study}

Initial water penetration results are shown in Figure 3-4. The rapid initial water penetration rate was found in the tablet prepared from the heat treatment excipient. Water penetrates the whole body of the tablet within 1 minute. For the wet granulated excipient, the surface of tablet showed a broken film structure, water penetrated through the broken parts of the film. The water penetrated to $1 / 3$ of the tablet within 1 minute. The slowest initial water penetration rate was obtained from the tablet prepared by spray dried coprocessed excipient. Water stayed primarily on the surface of the tablet and the body of the tablet appeared dry. This confirmed the finding from the tablet surface morphology study. The film-like surface structure prevents the water penetration thereby decreasing the drug release rate.

\subsubsection{The effect of co-processed excipients' formulation on drug release}

\subsubsection{The effect of the type of plasticizer}

The drug release rate was decreased while increasing the $\log \mathrm{P}$ value of plasticizer as illustrated on Figure 3-5. Since the similar porosity results was obtained for the tablets prepared by different plasticizers as shown in Table 3-3. The hydrophobicity of plasticizer plays a role for controlling the drug release. For hydrophilic plasticizers TEC and ATEC, they have some solubility in the water. They will dissolve in the water while in contact with the aqueous dissolution medium and leave the pores for water penetration and drug diffusion resulting in a rapid dissolution rate. DBS and ATBC are hydrophobic and practically insoluble in the water. The rate of these two hydrophobic plasticizers leaving the matrices is much slower than those two hydrophilic plasticizers. According to the glass transition temperature of CA with different plasticizers (Figure 2-4), ATBC has the better interaction with the CA compare to DBS. Therefore, ATBC remains in the matrices longer than DBS. Thus the slowest drug release rate was observed for the tablets containing ATBC as a plasticizer. 


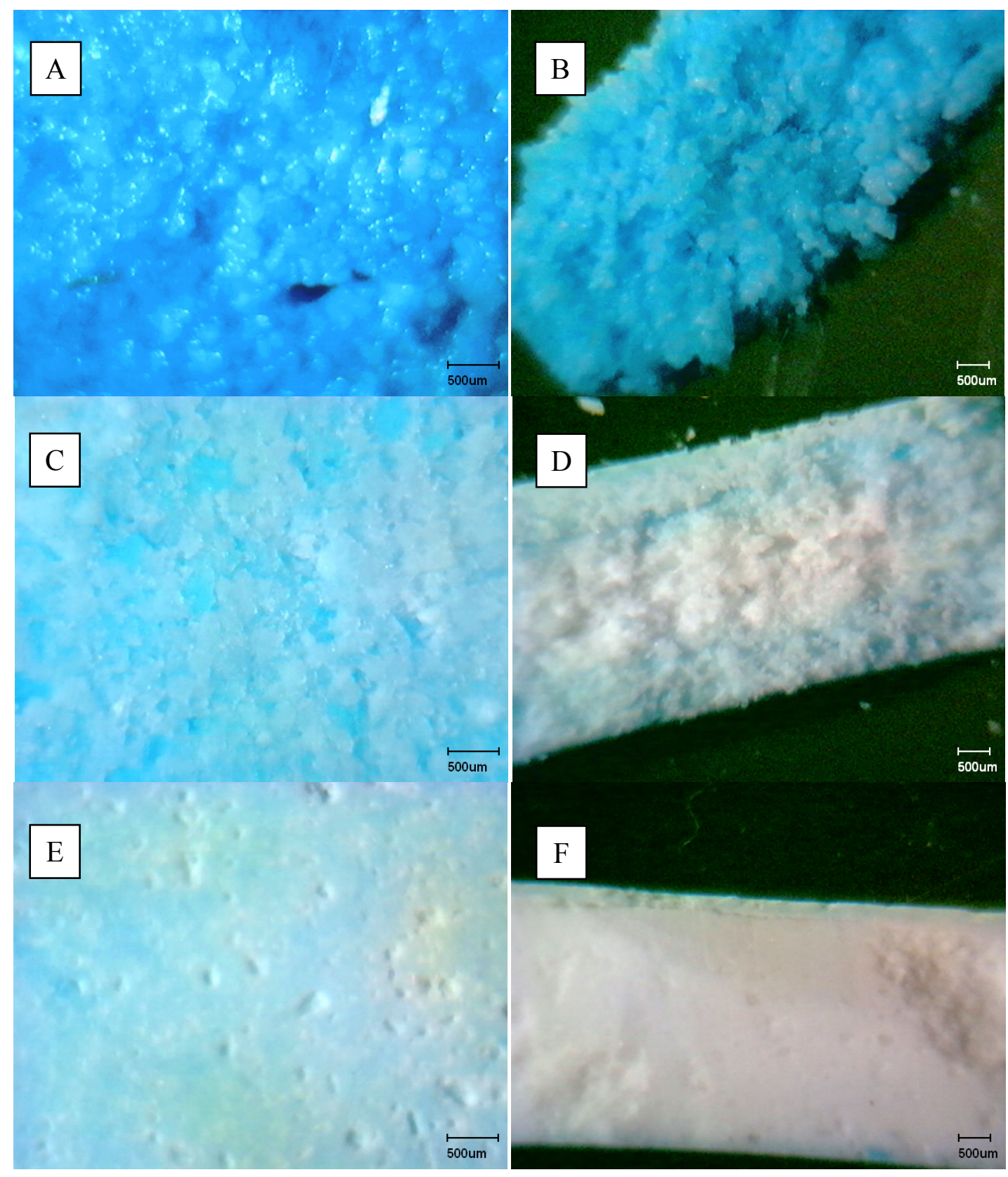

Figure 3-4. Initial water penetration study

A: Surface of tablet containing 20\% propranolol and $79.5 \%$ heat treated excipient F2; B: cross-section of tablet containing $20 \%$ propranolol and $79.5 \%$ heat treated excipient $\mathrm{F} 2$; C: surface of tablet containing $20 \%$ propranolol and $79.5 \%$ wet granulated excipient F2; D:tablets containing 20\% propranolol and $79.5 \%$ spray dried excipient F2. 


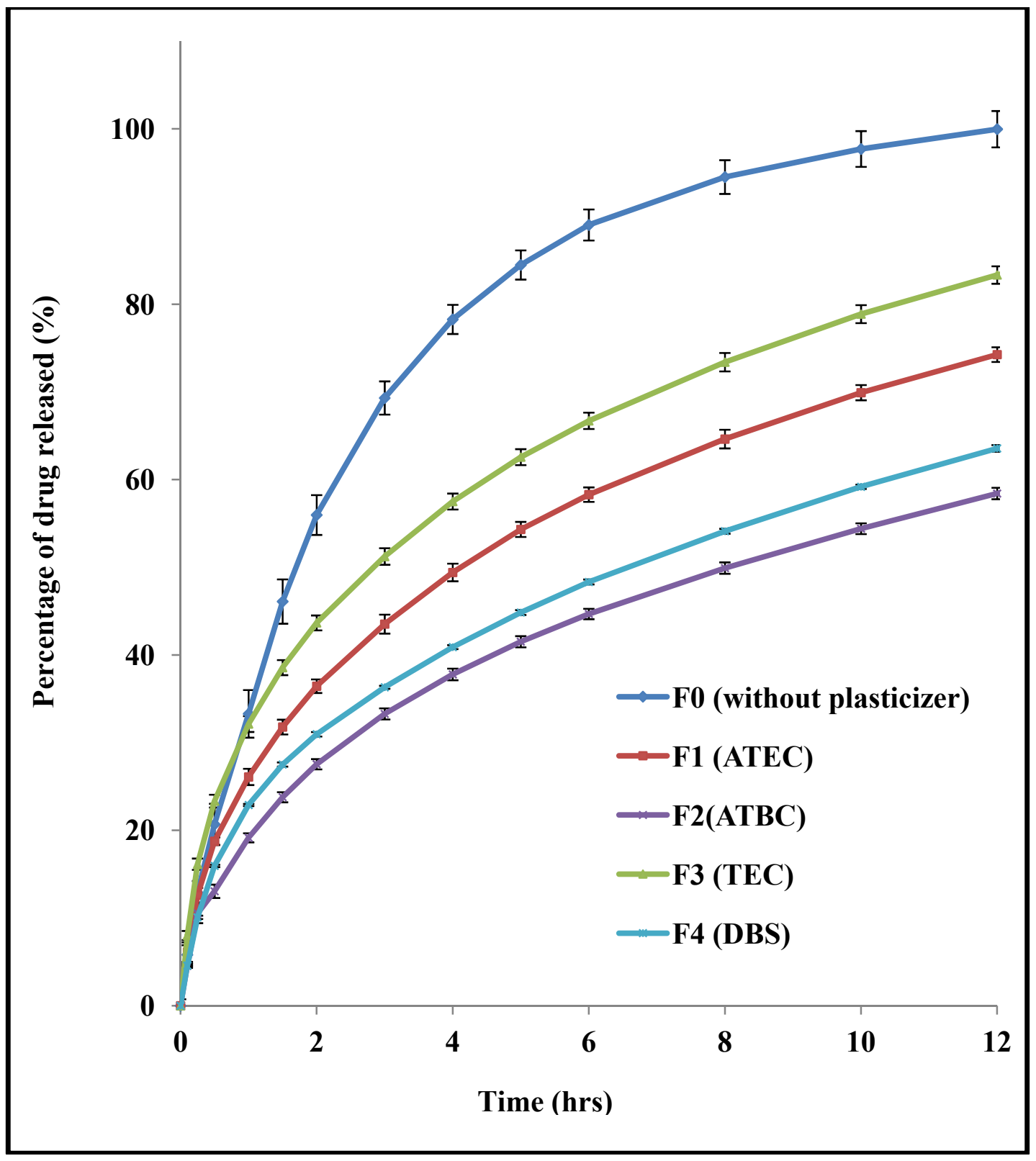

Figure 3-5. The effect of the type of plasticizer of co-processed excipient on the drug release 
Table 3-3. Porosity of tablets prepared by propranolol hydrochloride and coprocessed excipients with different plasticizers

\begin{tabular}{lc}
\hline Formulations & Porosity (\%) \\
\hline F0(no plasticizer) & $16.95 \pm 1.48$ \\
F1(ATEC) & $9.37 \pm 0.65$ \\
F2(ATBC) & $9.53 \pm 0.85$ \\
F3(TEC) & $10.05 \pm 0.88$ \\
F4(DBS) & $10.66 \pm 1.76$ \\
\hline
\end{tabular}




\subsubsection{The effect of plasticizer concentration}

Figure 3-6 demonstrates the concentration of plasticizer has a strong impact on the drug release rate. The drug dissolution rate was decreased with increasing hydrophobic plasticizer concentration. According to the Heckel's plot result (Figure 2-7), the plasticity of the granules was also increased with increasing concentration of plasticizer. Granules with better plasticity form more solid bonding during compression and lead to harder tablet at the same compression pressures. Therefore significant reduction of tablet porosity $(\mathrm{p}<0.05)$ was observed as shown in Table 3-4, also showing a difference in drug dissolution profile.

\subsubsection{The effect of compression force on drug dissolution}

One common feature of the hydrophobic matrix tablet is the compression force effect on drug dissolution. Tablets compressed at high compression force result in less volume and lower porosity. As a consequence, the drug release rate will be slower at the higher compression force. This phenomenon was observed in different hydrophobic matrix systems. In our study, the compression force effect on drug dissolution profile was investigated on the tablets containing $20 \%$ drug propranolol hydrochloride and spray dried co-processed excipient F2. The result is shown in Figure 3-7. It is evident that drug release rate decreased as compression force increased. The porosity of the tablets as shown in Table 3-5 confirmed the porosity decreased as the compression force increased. These results show typical hydrophobic matrix tablet behavior when using this novel coprocessed excipient as a retarding agent.

\subsection{5 $\mathrm{pH}$ effect of dissolution medium on drug dissolution profile}

In our study, $\mathrm{CA}$ is a $\mathrm{pH}$-independent polymer. It remains insoluble in GI tract $(\mathrm{pH}$ 1.2-7.4). The model drug propranolol hydrochloride is a hydrochloride salt with pka 9.5. When the $\mathrm{pH}$ ranges from 1.2 to 7.4 , it shows a $\mathrm{pH}$ independent solubility profile. The result of $\mathrm{pH}$ effect on drug dissolution was shown in Figure 3-8. The drug release rate in three different medium are very close to each other. We used the dissolution profile of water as reference, the $f_{2}$ value of the simulated gastric fluid (SGF) is 71.2 and the simulated intestinal fluid (SIF) is 95.7. The $f_{2}$ results suggest the three dissolution profiles are similar. Based on this result, we can conclude this novel co-processed excipient can provide a $\mathrm{pH}$ independent dissolution profile for a $\mathrm{pH}$ insensitive drug. However, for a $\mathrm{pH}$ sensitive drug, the dissolution profile is expected to vary under different $\mathrm{pH}$ conditions as the solubility of the drug changes.

\subsubsection{Drug solubility effect on drug dissolution profile}

Three drugs with different water solubility were selected in this study. The drug solubility in respect to the dissolution medium were tested and listed in Table 3-6. Based 


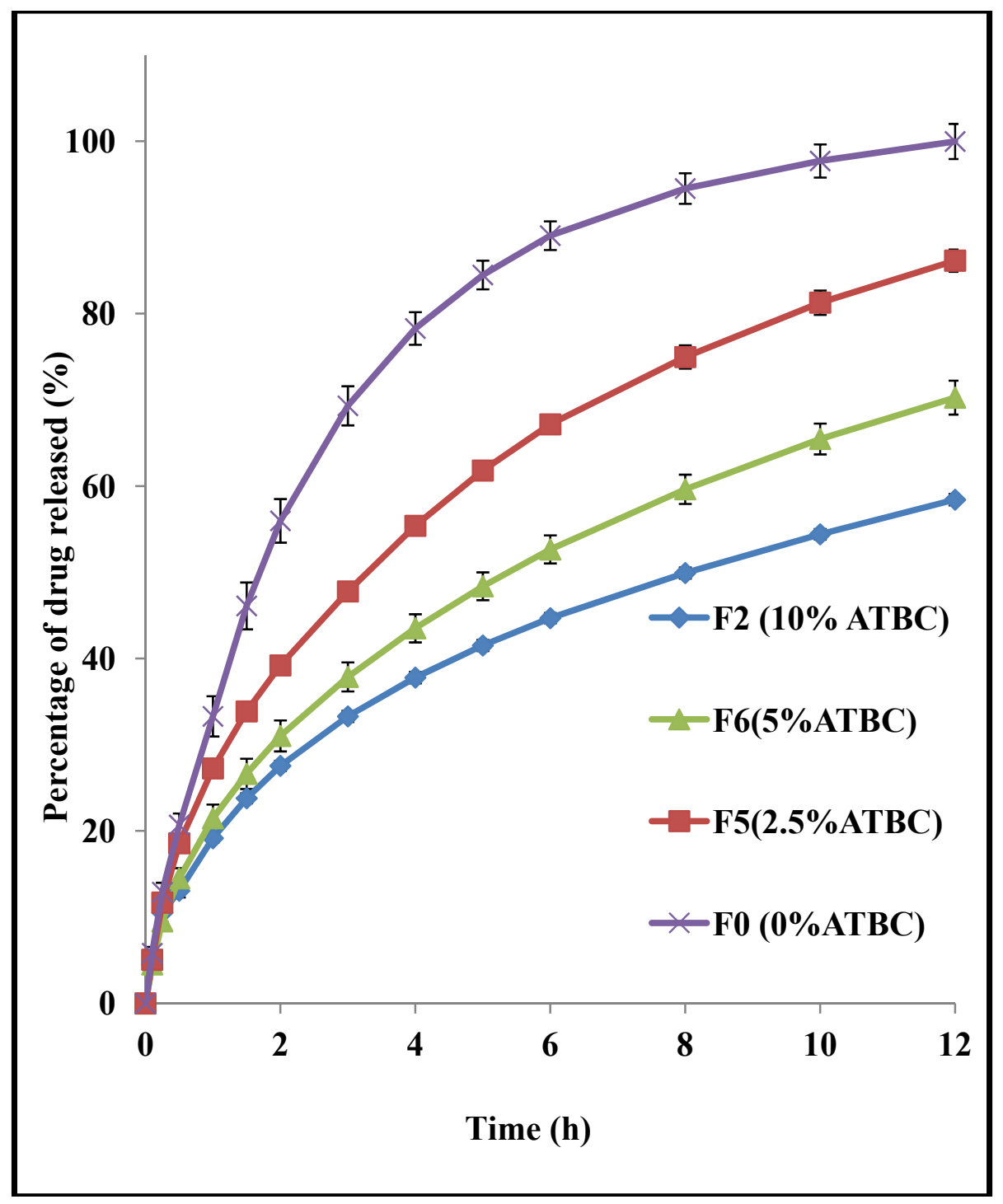

Figure 3-6. The effect of plasticizer concentration of co-processed excipient on the drug release 
Table 3-4. Porosity of tablets prepared by propranolol and co-processed excipients with different concentration of ATBC

\begin{tabular}{lc}
\hline Formulations & Porosity (\%) \\
\hline F0(no plasticizer) & $16.95 \pm 1.48$ \\
F5(2.5\%ATBC) & $15.63 \pm 1.06$ \\
F6 (5\% ATBC) & $12.35 \pm 0.84$ \\
F2(10\%ATBC) & $9.53 \pm 0.85$ \\
\hline
\end{tabular}




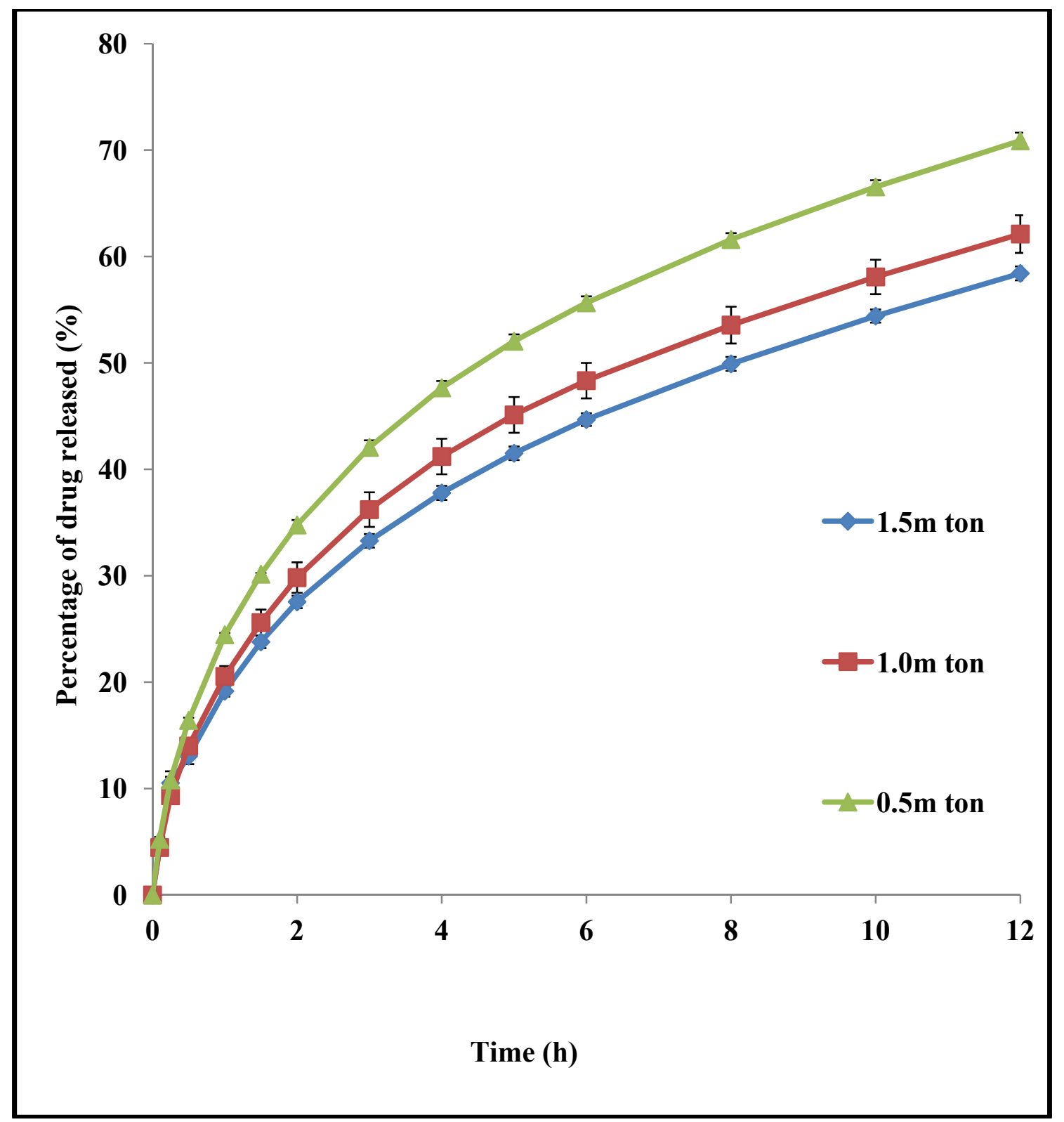

Figure 3-7. Compression force effect on drug release 
Table 3-5. Porosity of tablets prepared by propranolol and spray dried excipient F2 at different compression force

\begin{tabular}{lc}
\hline Compression force & Porosity (\%) \\
\hline $0.5 \mathrm{~m}$ ton & $14.63 \pm 1.26$ \\
$1.0 \mathrm{~m}$ ton & $10.97 \pm 0.63$ \\
$1.5 \mathrm{~m}$ ton & $9.53 \pm 0.85$ \\
\hline
\end{tabular}




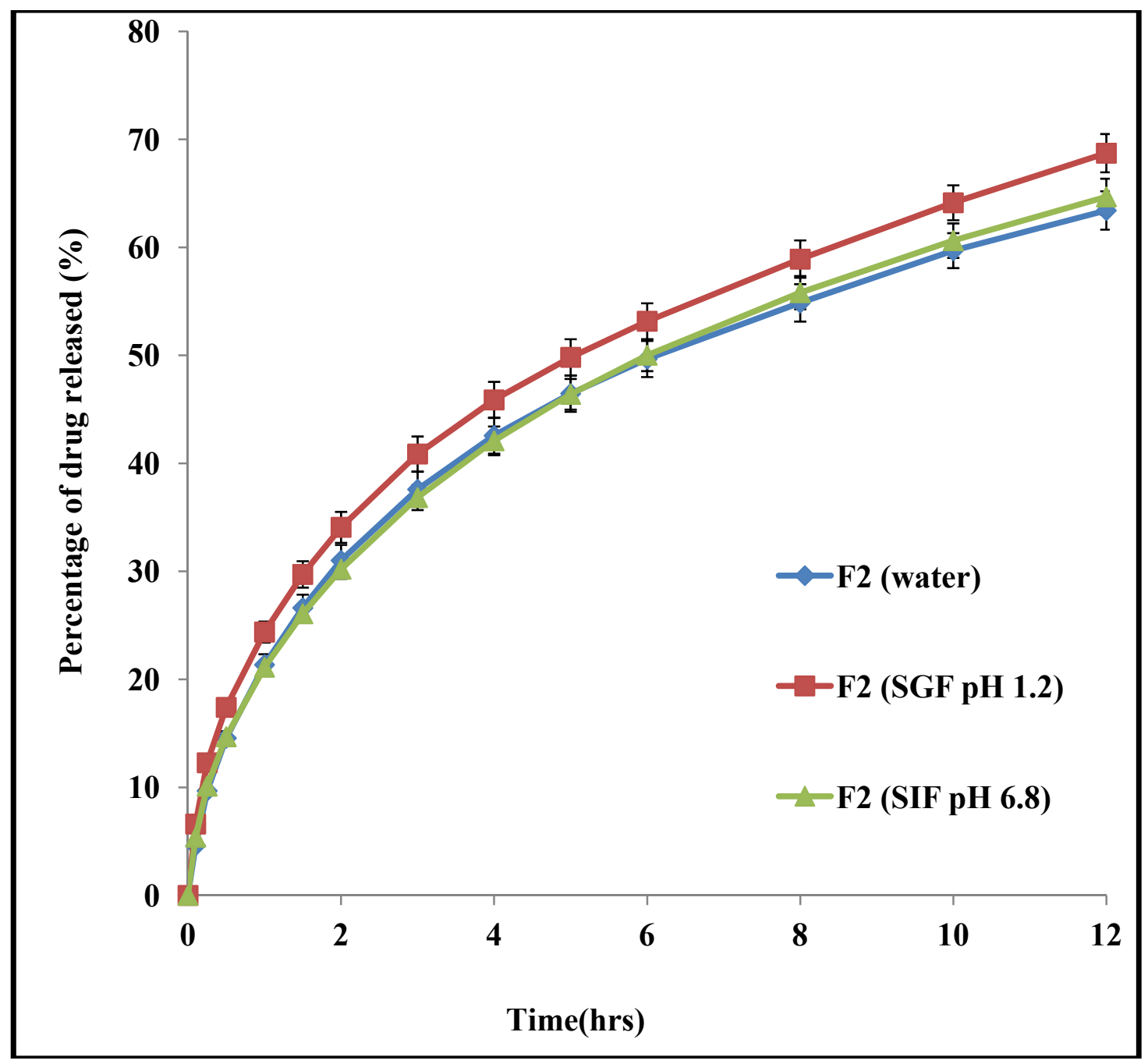

Figure 3-8. pH effect on drug release profile 
Table 3-6. Solubility of different drugs in dissolution medium at $37^{\circ} \mathrm{C}$

\begin{tabular}{lcc}
\hline Drug & Dissolution medium & Solubility (mg/ml) \\
\hline Propranolol hydrochloride & DI water & $192.79 \pm 1.93$ \\
Theophylline anhydrous & pH 7.4 phosphate buffer & $11.19 \pm 0.18$ \\
Flurbiprofen & pH 7.2 phosphate buffer & $4.52 \pm 0.07$ \\
\hline
\end{tabular}


on the solubility result, propranolol hydrochloride was used as a freely water soluble drug; theophylline anhydrous was used as sparingly soluble drug and flurbiprofen was used as water insoluble drug. The solubility effect on drug dissolution profile was illustrated in Figure 3-9. For all the co-processed excipients selected in this study, the drug with higher solubility always show faster release rate. For the same drug, the order of the release rate is F0>F1>F2. This order is valid for all three drugs. This result indicates the plasticized co-processed excipient has better control release ability than the unplasticized co-processed excipient. However, the difference of the drug release rate between the plasticized excipient and unplasticized excipient for three drugs is not the same. The freely soluble drug propranolol hydrochloride exhibits a large difference (more than $40 \%$ release difference in 12 hours) in dissolution profile between the plasticized formulation and unplasticized formulation. While there is a small amount decrease (less than $10 \%$ release difference in 12 hours) of plasticized formulation for the water insoluble drug flurbiprofen was observed. This result suggests the plasticizer effect has more impact when utilized on a water soluble drug.

\subsubsection{Drug release mechanism study}

\subsubsection{Higuchi model}

The Higuchi model was used to plot the drug release profile of the tablets with three different drugs and two different spray dried co-processed excipients. The results were displayed in Figures 3-10 and 3-11. All six formulations showed a good fit $\left(\mathrm{R}^{2}\right.$ $>0.98$ ) for the Higuchi model. This indicates the drug release from the novel coprocessed excipient matrix follows square root kinetics. The constant $\mathrm{K}$ which is the slope in the Higuchi equation represents the drug release rate. A higher $\mathrm{K}$ value was observed for unplasticized matrices (F0) in spite of the solubility of the drug. The drug with higher solubility also showed a higher $\mathrm{K}$ value for both plasticized and unplasticized matrices.

Typically, two factors are responsible for reducing the drug diffusion coefficient in the matrix. One is the porosity factor $\varepsilon$. Since the drug cannot cross the solid matrix, the void space available for drug diffusion is reduced by $1 / \varepsilon$. Another is tortuosity factor $\tau$. The pores structure in the matrix does not tend to be straight and the drug need to travel longer distance in order to diffuse. The diffusion coefficient is reduced by the increasing path length which is a tortuosity factor $\tau$. The effective diffusion coefficient from the matrix considered both factors and is defined as:

$$
\mathrm{D}_{\mathrm{eff}}=\mathrm{D} \varepsilon / \tau
$$

where $\mathrm{D}_{\text {eff }}$ is the effective diffusion coefficient; $\mathrm{D}$ is diffusion coefficient. According the Higuchi equation, after we know the $\mathrm{K}, \mathrm{C}_{\mathrm{s}}, \mathrm{C}_{0}$ and $\varepsilon, \mathrm{D}_{\text {eff }}$ can be calculated. $\mathrm{D}_{\text {eff }}$ describes the real situation for drug diffusion from the matrix [82]. 


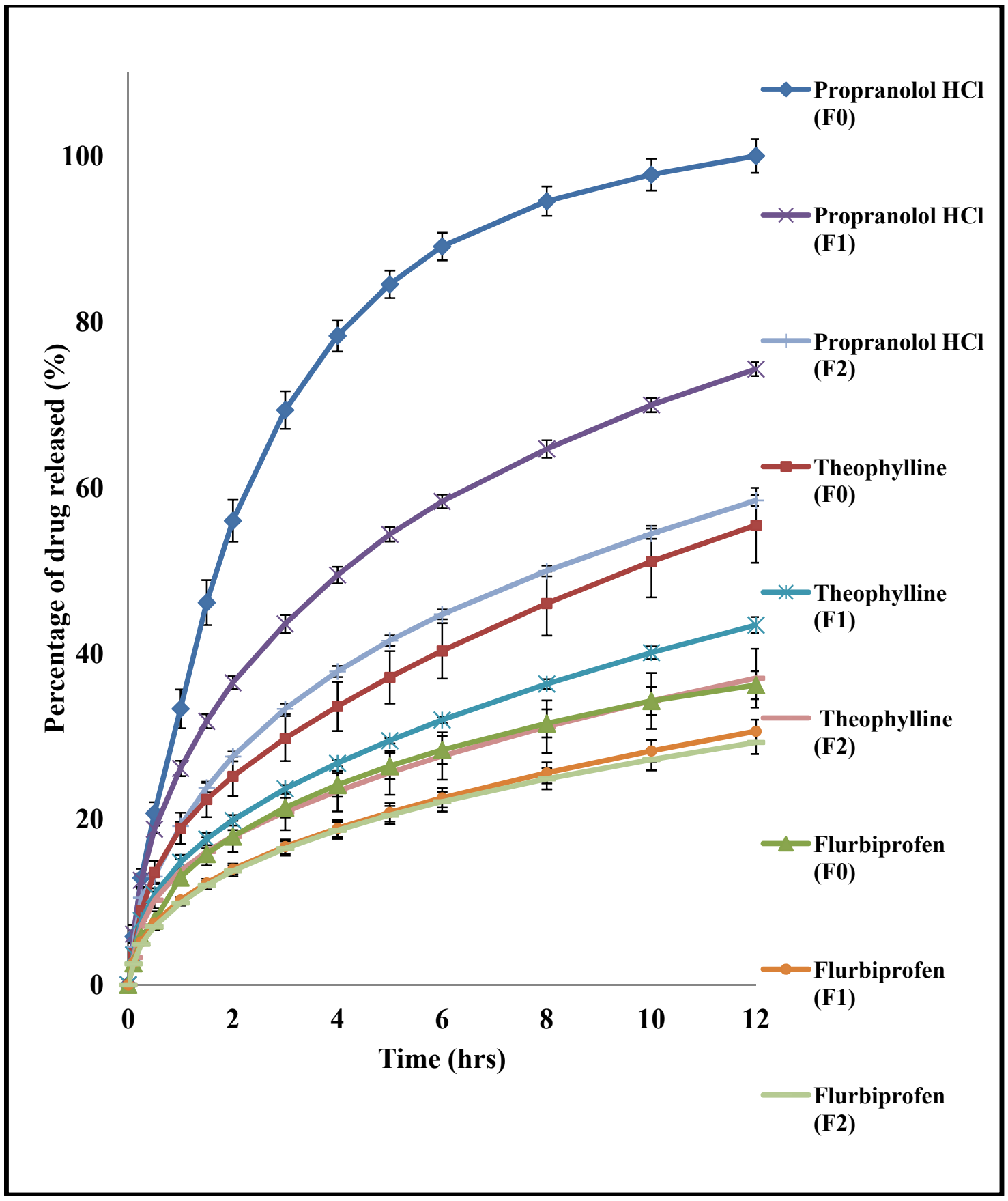

Figure 3-9. Drug solubility effect on dissolution profile 


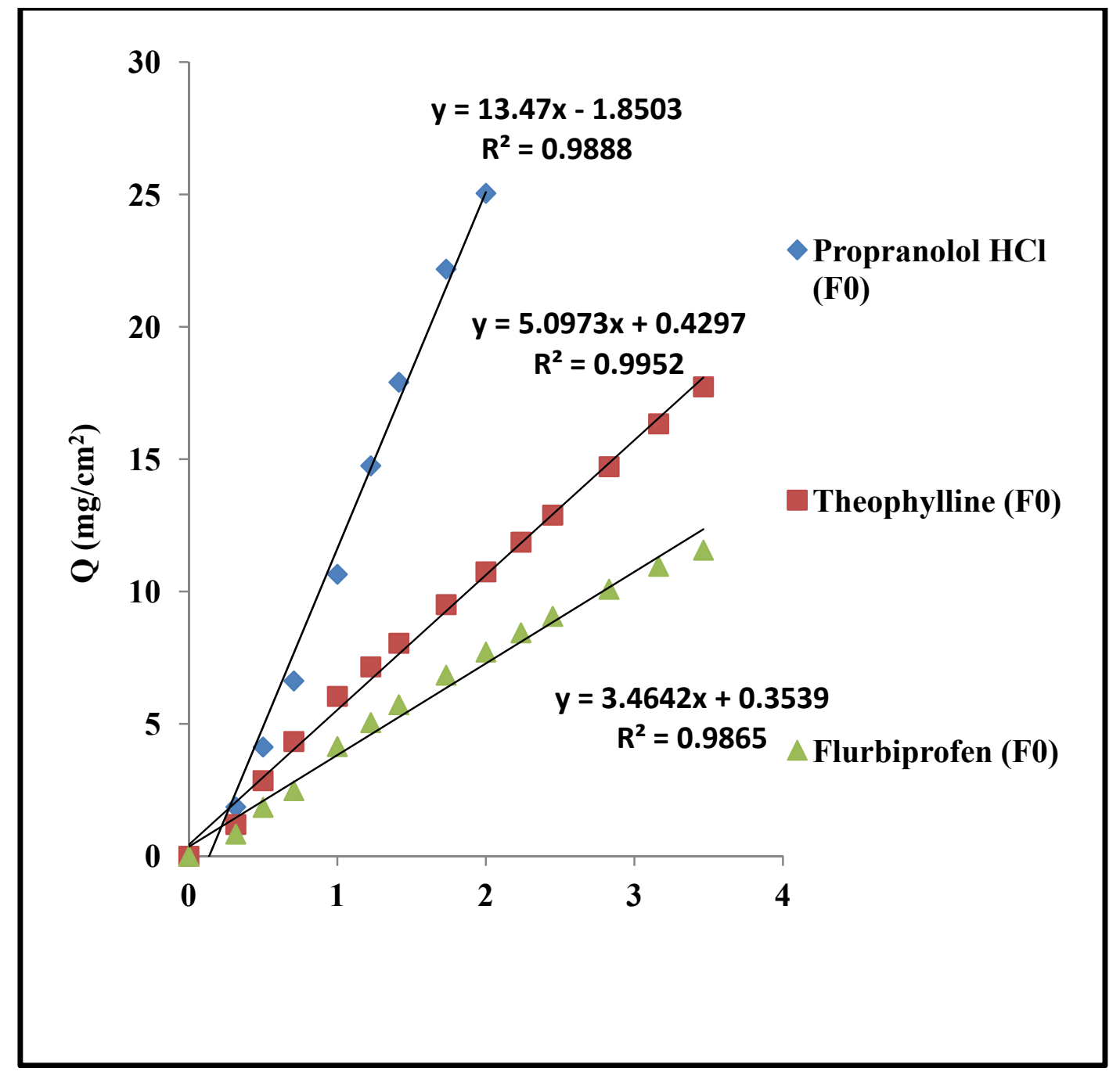

Figure 3-10. Higuchi model fitting for tablets prepared from co-processed excipient F0 


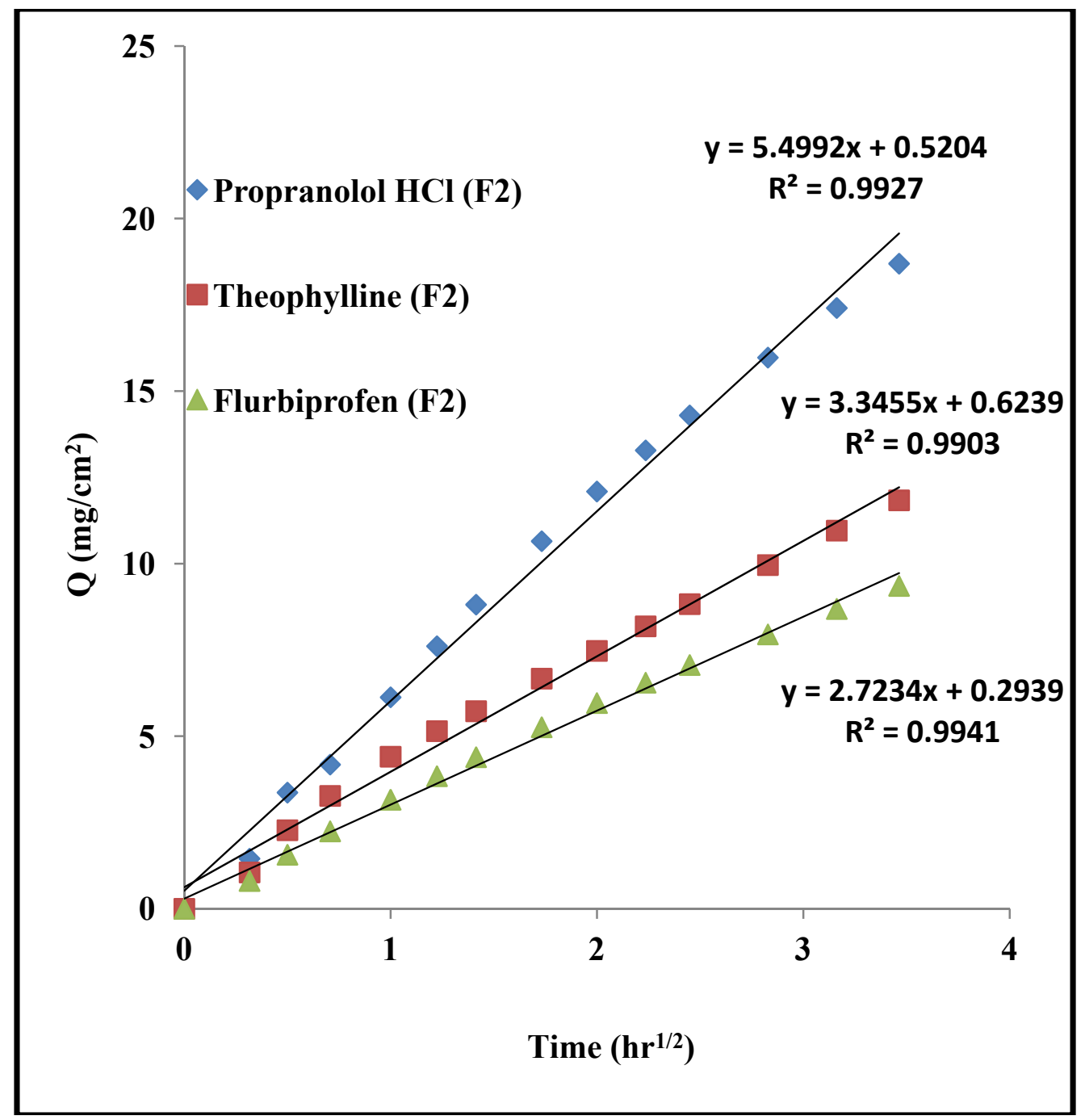

Figure 3-11. Higuchi model fitting for tablets prepared from the co-processed excipient F2 
The $\mathrm{D}_{\text {eff }}$ for six different matrices was calculated and listed in Table 3-7. For all three drugs, the $\mathrm{D}_{\text {eff }}$ in an unplasticized matrix (F0) is higher than in a plasticized matrix (F2). This indicates the presence of the plasticizer can help to prevent the drug diffusion from the matrix.

\subsubsection{Power law model}

The same six dissolution profiles were fitted to the logarithmic transformed power law equation. The results were illustrated in Figures 3-12 and 3-13. The $n$ value which is the slope of logarithm transformed power law equation indicates the drug release mechanism. For all the tablets prepared by the spray dried co-processed excipients F2, $n$ values for all drugs are less or close to 0.45 . This suggests drug release is controlled by Fickian diffusion. For the tablets prepared by the spray dried co-processed excipient F0, $n$ values for water insoluble drug flurbiprofen and sparingly water soluble drug theophylline are less or close to 0.45 . But $\mathrm{n}$ value for water soluble drug propranolol hydrochloride is 0.621 , which suggests both drug diffusion and matrix relaxation take place.

For the hydrophobic matrix tablet, the tablet will remain intact after dissolution. Since the polymer CA is water insoluble and does not hydrate in the water, the size change of matrix tablet during dissolution was not expected. Table 3-8 listed the tablet size change after the dissolution. Except for the tablet containing propranolol hydrochloride and spray dried F0, other tablets all maintained the similar size after dissolution. This confirmed with the result of the power law equation that tablet relaxation did not contribute significantly to the drug release. For the tablet comprising freely water soluble drug and unplasticized excipient, a $13.35 \%$ increase in thickness was observed. The picture taken after dissolution (Figure 3-14B) showed the several cracks in the body of tablet. This cracks can shorten the drug diffusion pathway, therefore increase the drug release rate. These cracks also contribute to the matrix relaxation mechanism. Because we did not observe the matrix relaxation in other 5 formulations, it can be concluded this is not caused by the polymer relaxation. The possible reason maybe the high osmotic pressure generated by the freely water soluble drug propranolol hydrochloride affected the tablet structure and caused the resulting cracks. However for the tablet prepared with propranolol hydrochloride and plasticized co-processed excipient F2 the plasticized tablet is more robust and can withstand the osmotic pressure and the tablet structure can be maintained. For the water soluble drug, the plasticizer can maintain tablet shape during dissolution, therefore prolonging the drug release.

\subsection{Conclusions}

The spray dried co-processed excipients successfully controlled the release of a freely water soluble drug from a matrix tablet. It demonstrated a slower drug release profile than the co-excipients prepared by the other two processing methods. The surface morphology and initial water penetration study found the spray dried co-processed 
Table 3-7. Higuchi release constant and effective diffusion coefficient for different drugs in different matrices

\begin{tabular}{lcc}
\hline Formulations & K value & D $_{\text {eff }}$ \\
\hline Propranolol (F0) & 13.47 & 0.00739 \\
Theophylline (F0) & 5.0973 & 0.014697 \\
Flurbiprofen (F0) & 3.4642 & 0.016688 \\
Propranolol (F2) & 5.4992 & 0.001108 \\
Theophylline (F2) & 3.3455 & 0.006296 \\
Flurbiprofen (F2) & 2.7234 & 0.010293 \\
\hline
\end{tabular}




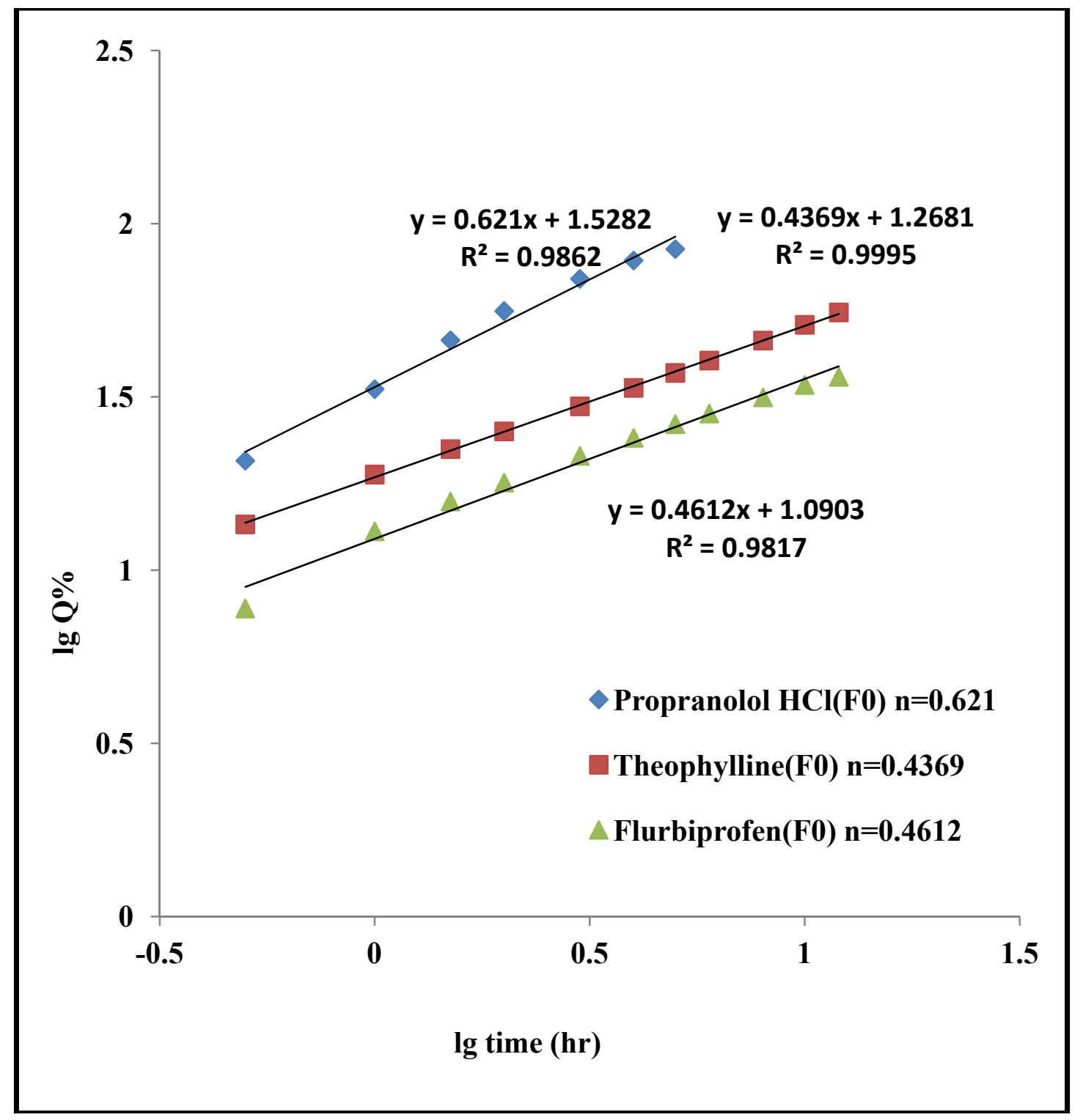

Figure 3-12. Power law model fitting for tablets prepared from the co-processed excipient F0 


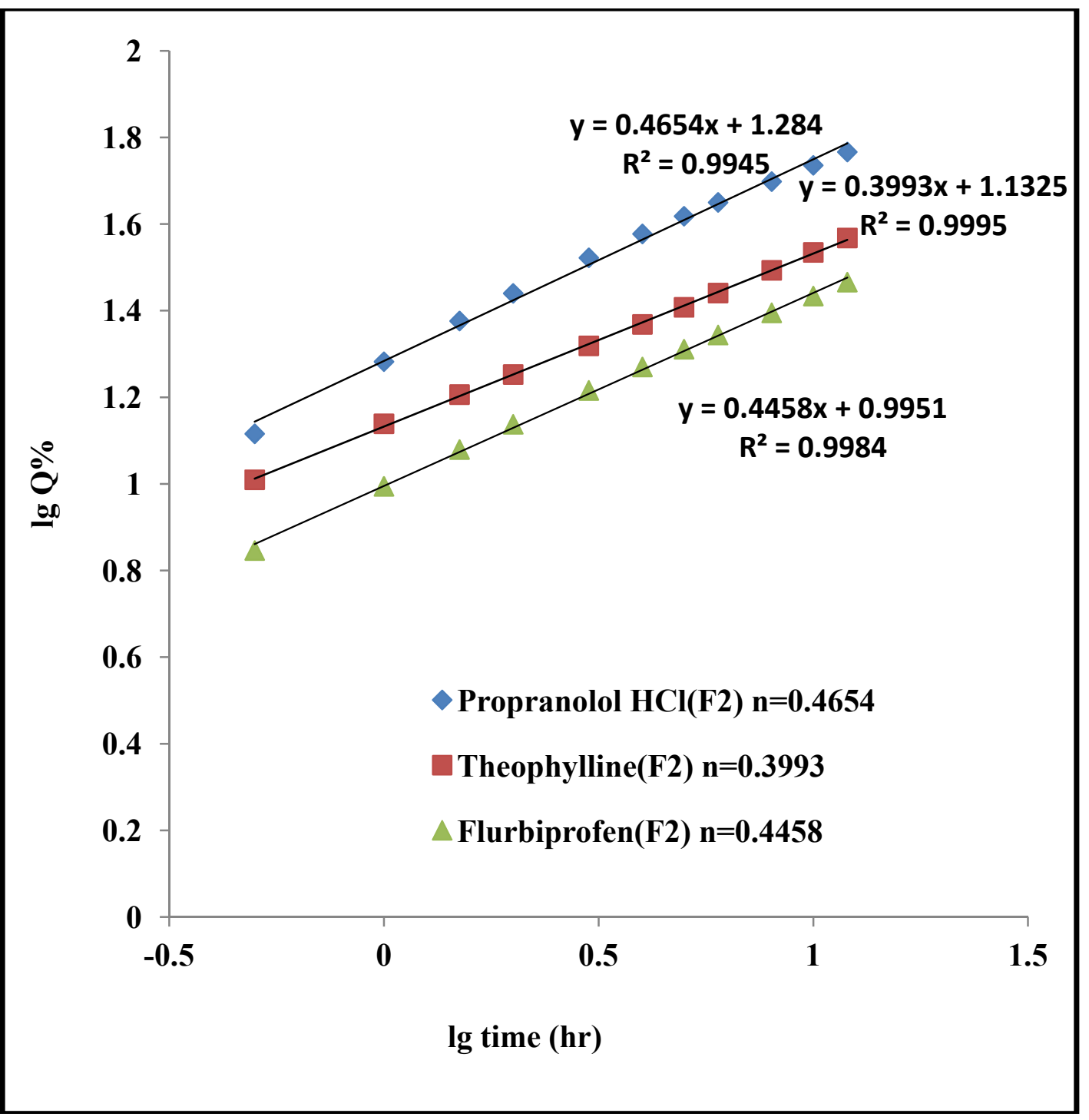

Figure 3-13. Power law model fitting for tablets prepared from the co-processed excipient $\mathbf{F} 2$ 
Table 3-8. Tablet size change after dissolution

\begin{tabular}{lcc}
\hline Formulations & Diameter change (\%) & Thickness change(\%) \\
\hline Propranolol (F0) & $2.65 \pm 0.3$ & $13.35 \pm 0.43$ \\
Theophylline (F0) & $1.57 \pm 1.31$ & $0.66 \pm 0.01$ \\
Flurbiprofen (F0) & $2.09 \pm 1.23$ & $0.29 \pm 0.21$ \\
Propranolol (F2) & $0.18 \pm 0.32$ & $0.28 \pm 0.57$ \\
Theophylline (F2) & $0.10 \pm 0.10$ & $0.66 \pm 0.16$ \\
Flurbiprofen (F2) & $0.76 \pm 0.26$ & $0.19 \pm 0.42$ \\
\hline
\end{tabular}




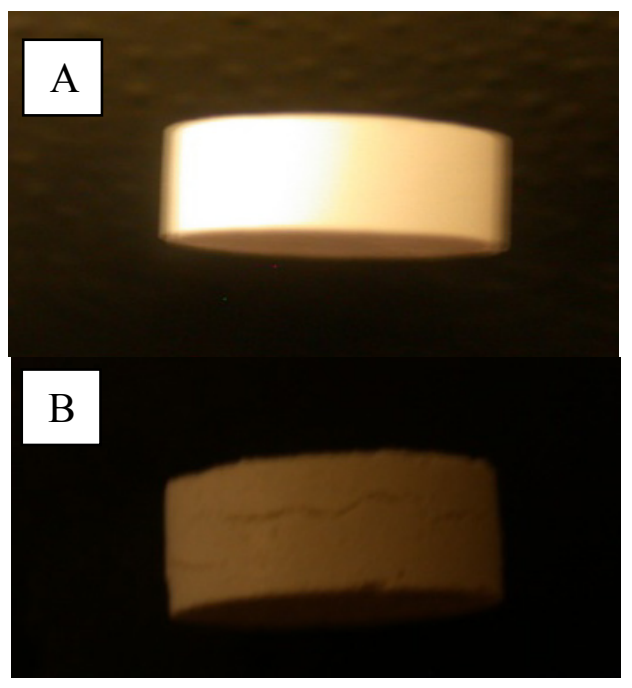

Figure 3-14. Pictures of tablet containing 20\% propranolol hydrochloride and spray dried excipient $F 0$

A: Before dissolution; B: after 12 hours dissolution. 
excipient can form the continuous film liked structure on the tablet surface which can effectively prevent water penetration and drug diffusion. The drug release rate from the matrix tablets containing the novel spray dried excipients can be decreased by increasing the hydrophobicity of the plasticizer and plasticizer concentration. The drug release rate from the matrix was affected by the compression force but not the $\mathrm{pH}$ of the dissolution medium. The drug release from the matrix tablet can be described by Higuchi equation and the power law equation. Based on porosity results and drug release mechanism study, addition of plasticizer to the co-processed excipients showed decrease in the drug release rate is due to three reasons: a) decrease in the porosity of the tablet; b) decrease in the effective diffusion coefficient; c) assist in maintaining tablet structure during dissolution. 


\section{Chapter 4. Hydrophilic Polymer Effect on the Plasticized Cellulose Acetate Matrix Tablets}

\subsection{Introduction}

For a highly water soluble drug, the use of hydrophilic polymer alone for controlling the drug release from the matrix is restricted due to the rapid diffusion through the gel layer. For such drugs, hydrophobic polymers are suitable as retarding agents. The hydrophobic polymers can offer several advantages including good stability at varying $\mathrm{pH}$ and resistant to the environmental moisture [2, 17, 19] However, for a simple monolithic hydrophobic matrix, an increase of the diffusion pathway and a decrease of the effective diffusion area as a function of time often results in incomplete release within the gastrointestinal transit time. To modulate drug release, it may be necessary to incorporate soluble ingredients such as lactose or PEG into formulation. The presence of soluble ingredient in the formulations helps to form numerous pores in hydrophobic matrix network for water penetration and drug diffusion. Therefore, complete drug release can be obtained from the highly porous matrix [44]. But burst release is a potential risk when an excessive amount of water soluble ingredient is present in the hydrophobic matrix. The hydrophilic nature of these pore formers can result the rapid surface erosion and cause fast surface drug release initially.

In this chapter, we developed a swellable porous matrix tablet by incorporating a small amount of hydrophilic polymer into the hydrophobic matrix. The swelling property of the hydrophilic polymer during dissolution is utilized to generate highly porous structure in the hydrophobic matrix for obtaining complete drug release. The initial viscous gel formation of the hydrophilic polymer upon contacting with water also can help to suppress the burst release of the drug. The effect of the type of hydrophilic polymer, the concentration of hydrophilic polymer, the viscosity of hydrophilic polymer and the particle size of hydrophilic polymer on drug release and the drug release mechanism from this novel system was investigated in this chapter.

\subsection{Materials and Methods}

\subsubsection{Materials}

Cellulose acetate with 39.8\% acetyl content and $38 \mathrm{cp}$ viscosity (CA 398-10 NF, Eastman, Kingsport, TN) was used as the main polymer in this study. Triethyl citrate (TEC), acetyltriethyl citrate (ATEC), acetyltributyl citrate (ATBC) and dibutyl sebacate (DBS) (Morflex Inc., Greensboro, NC) were used as plasticizers. Milled dicalcium phosphate dihydrate (milled DCP Innophos Inc., Cranbury, IL) was used as filler. Magnesium stearate (Mallinckrodt, Paris, KY) was used as a lubricant. Spray dried lactose monohydrate (Lactose 316, Foremost, Baraboo, WI) and partially pregelatinized corn starch $\left(\operatorname{Starch}{ }^{\circledR} 1500\right.$, Colorcon, West point, PA) were used as pore former for 
hydrophobic matrix tablet. Propranolol hydrochloride was used as a water soluble model drug. Five different viscosity grades (E5, E50, K100 CR, K4M CR and K100M CR) of hydroxypropyl methylcellulose (HPMC, Dow Chemical Company, Midland MI), the highest viscosity grade of hydroxypropyl cellulose (HPC HXF, Asland, Covington, KY), hydroxyethyl cellulose (HEC HHX, Asland, Covington, KY) and polyethylene oxide (Polyox® 303, Dow Chemical, Midland MI) were selected as hydrophilic polymer. Methylene blue (JT Baker, Phillipsburg, NJ) and FD\&C orange (Opatint PG, Colorcon, West point, PA) were used as dye to indicate the different components in the tablets.

\subsubsection{Methods}

\subsubsection{Tablets preparation}

The tablet formulations were listed in Tables 4-1 to 4-6. The tablets were prepared by direct compression method. All the ingredients were mixed by passing through 20 mesh sieve 3 times. The mixtures were compressed on a Carver Press (Carver Inc., Wabash, IN) by $3 / 8$ inch flat face punch at 1.5 metric ton compression force. The dwell time is 3 seconds and the target weight is $400 \mathrm{mg}$.

\subsubsection{In vitro dissolution study}

The USP Apparatus II (Hanson SR-8 Plus, Hanson Research Corporation, Chatsworth, CA) was used to evaluate the drug release. Three tablets from each batch were tested. $900 \mathrm{ml}$ of distilled water at $37 \pm 0.5^{\circ} \mathrm{C}$ was used as the dissolution medium for evaluate the release of propranolol hydrochloride. The samples were taken at predetermined time points for 12 hours. The absorbance of the solution was measured at $289 \mathrm{~nm}$ by a UV-spectrophotometer (Perkin-Elmer Corporation, Norwalk, CT). The drug concentration and the percentage of drug release were calculated by the software DissLab®.

The difference of drug dissolution profiles was evaluated by using $f_{2}$ value as described in Equation 4-1 [24].

$$
f_{2}=50 \times \log \left\{\left[1+(1+1 / \mathrm{n}) \sum_{\mathrm{t}=1}\left(\mathrm{R}_{\mathrm{t}}-\mathrm{T}_{\mathrm{t}}\right)^{2}\right]^{-0.5} \times 100\right\}
$$

where $f_{2}$ is a similarity factor, $\mathrm{n}$ is the number of sample times, $\mathrm{R}_{\mathrm{t}}$ is the average percentage of drug released at time $t$ for the reference sample, $T_{t}$ is the average percentage of drug released at time $t$ for the test sample. According to FDA's guidance for industry, if $f_{2}$ values were greater than 50 , the two dissolution profiles can be consider as similar or equivalent. 
Table 4-1. Formulations for tablets prepared with different type of pore formers

\begin{tabular}{lcccc}
\hline \multirow{2}{*}{ Ingredients } & \multicolumn{4}{c}{ Percentage (\%) } \\
\cline { 2 - 5 } & H0 & H2 & P1 & P2 \\
\hline Propranolol hydrochloride & 20 & 20 & 20 & 20 \\
Spray dried excipient F2 & 79.5 & 74.5 & 74.5 & 74.5 \\
Polyox ${ }^{\circledR} 303$ & 0 & 5 & 0 & 0 \\
Lactose 316 & 0 & 0 & 5 & 0 \\
Starch ${ }^{\circledR} 1500$ & 0 & 0 & 0 & 5 \\
Magnesium Stearate & 0.5 & 0.5 & 0.5 & 0.5 \\
Total & 100 & 100 & 100 & 100 \\
\hline
\end{tabular}

Table 4-2. Formulations for tablets prepared with different concentrations of hydrophilic polymer

\begin{tabular}{lcccc}
\hline \multirow{2}{*}{ Ingredients } & \multicolumn{4}{c}{ Percentage (\%) } \\
\cline { 2 - 5 } & H0 & H1 & H2 & H3 \\
\hline Propranolol hydrochloride & 20 & 20 & 20 & 20 \\
Spray dried excipient F2 & 79.5 & 77 & 74.5 & 69.5 \\
Polyox ${ }^{\circledR} 303$ & 0 & 2.5 & 5 & 10 \\
Magnesium Stearate & 0.5 & 0.5 & 0.5 & 0.5 \\
Total & 100 & 100 & 100 & 100 \\
\hline
\end{tabular}


Table 4-3. Formulations for tablets prepared with different hydrophilic polymers

\begin{tabular}{lcccc}
\hline \multirow{2}{*}{ Ingredients } & \multicolumn{4}{c}{ Percentage (\%) } \\
\cline { 2 - 5 } & H2 & H4 & H5 & H6 \\
\hline Propranolol hydrochloride & 20 & 20 & 20 & 20 \\
Spray dried excipient F2 & 74.5 & 74.5 & 74.5 & 74.5 \\
Polyox 303 & 5 & 0 & 0 & 0 \\
HPMC K100M CR & 0 & 5 & 0 & 0 \\
HPC HXF & 0 & 0 & 5 & 0 \\
HEC HHX & 0 & 0 & 0 & 5 \\
Magnesium Stearate & 0.5 & 0.5 & 0.5 & 0.5 \\
Total & 100 & 100 & 100 & 100 \\
\hline
\end{tabular}

Table 4-4. Formulations for tablets prepared with different viscosity grades of hydrophilic polymer

\begin{tabular}{lllccc}
\hline \multirow{2}{*}{ Ingredients } & \multicolumn{5}{c}{ Percentage (\%) } \\
\cline { 2 - 6 } & H7 & H8 & H9 & H10 & H4 \\
\hline Propranolol hydrochloride & 20 & 20 & 20 & 20 & 20 \\
Spray dried excipient F2 & 74.5 & 74.5 & 74.5 & 74.5 & 74.5 \\
HPMC E5 & 5 & 0 & 0 & 0 & 0 \\
HPMC E50 & 0 & 5 & 0 & 0 & 0 \\
HPMC K100LV & 0 & 0 & 5 & 0 & 0 \\
HPMC K4M & 0 & 0 & 0 & 5 & 0 \\
HPMC K100M & 0 & 0 & 0 & 0 & 5 \\
Magnesium Stearate & 0.5 & 0.5 & 0.5 & 0.5 & 0.5 \\
Total & 100 & 100 & 100 & 100 & 100 \\
\hline
\end{tabular}


Table 4-5. Formulations for tablets prepared with different co-processed excipients and hydrophilic polymer Polyox 303

\begin{tabular}{lccc}
\hline \multirow{2}{*}{ Ingredients } & \multicolumn{3}{c}{ Percentage (\%) } \\
\cline { 2 - 4 } & H2A & H2B & H2C \\
\hline Propranolol hydrochloride & 20 & 20 & 20 \\
Spray dried excipient F2 & 74.5 & 74.5 & 74.5 \\
Polyox ${ }^{\circledR} 303(20-60$ mesh) & 5 & 0 & 0 \\
Polyox ${ }^{\circledR} 303(60-140$ mesh) & 0 & 5 & 0 \\
Polyox 303 (<140mesh) & 0 & 0 & 5 \\
Magnesium Stearate & 0.5 & 0.5 & 0.5 \\
Total & 100 & 100 & 100 \\
\hline
\end{tabular}

Table 4-6. Formulations for tablets prepared with different co-processed excipients and hydrophilic polymer Polyox 303

\begin{tabular}{lccc}
\hline \multirow{2}{*}{ Ingredients } & \multicolumn{3}{c}{ Percentage (\%) } \\
\cline { 2 - 4 } & H11 & H12 & H2 \\
\hline Propranolol hydrochloride & 20 & 20 & 20 \\
Spray dried excipient F0 & 74.5 & 0 & 0 \\
Spray dried excipient F1 & 0 & 74.5 & 0 \\
Spray dried excipient F2 & 0 & 0 & 74.5 \\
Polyox® 303 & 5 & 5 & 5 \\
Magnesium Stearate & 0.5 & 0.5 & 0.5 \\
Total & 100 & 100 & 100 \\
\hline
\end{tabular}




\subsubsection{Optical microscopic examination}

In order to identify different components of tablet during dissolution, the colored tablets were prepared. An appropriate amount of methylene blue was added to the coprocessed excipient formulation in $0.1 \%$ concentration. The methylene blue was processed along with the other excipient as the same condition. The final co-processed excipient appeared a dark blue color. An appropriate amount of FD\&C orange dye was dispersed in the isopropanol alcohol, and then mixed with Polyox ${ }^{\circledR}$ 303. The wet mass was kept in $40^{\circ} \mathrm{C}$ oven overnight to remove the solvent. The dried particles were passed through 20 mesh sieve to obtain orange color Polyox ${ }^{\circledR}$ 303. The colored tablets were prepared by blue co-processed excipient F2, orange Polyox ${ }^{\circledR} 303$, white model drug propranolol hydrochloride and lubricant as the same concentration and same preparation procedure as the formulation $\mathrm{H} 0$ and $\mathrm{H} 2$. These colored tablets were placed in the same dissolution condition as the normal tablets for 1, 6 and 12 hours, and then took it out to observe the surface and cross-section under the Olympus MIC-D microscope. (Olympus, Center Valley, PA). The geometric dimension of the tablets was measured by using the caliper.

\subsubsection{Mathematic model fitting}

\subsection{Power law equation}

Drug-release mechanism was first studied by fitting the dissolution data to the power law equation [45].

$$
\mathrm{M}_{\mathrm{t}} / \mathrm{M}_{\infty}=\mathrm{k}^{*} \mathrm{t}^{\mathrm{n}}
$$

where $\mathrm{M}_{t}$ is cumulative amount of drug released at time $t, M \infty$ is cumulative amount of drug released at infinite time; $\mathrm{k}$ is release constant incorporating structural and geometric characteristics of the system, and $\mathrm{n}$ is the release exponent indicating the mechanism of drug release.

The release exponent ' $n$ ' in the power law takes multiple values based on geometry of the system. For a cylinder shape tablets, if $n<0.45$, the major release mechanism is Fickian diffusion; if the $\mathrm{n}$ value $>0.89$, Case-II transport in which the polymer relaxation is the rate limiting process; if $\mathrm{n}$ value between 0.45 and 0.89 , the "anomalous transport" takes place where both of drug diffusion and swelling of the polymer are involved.

\subsection{Peppas and Shalin equation}

Peppas and Shalin equation [50] is a modification of power law equation. It was performed by decoupling diffusion and "Case-II transport" with the following expression: 


$$
\mathrm{M}_{\mathrm{t}} / \mathrm{M}_{\infty}=\mathrm{k}_{1} * \mathrm{t}^{\mathrm{m}}+\mathrm{k}_{2} * \mathrm{t}^{2 \mathrm{~m}}
$$

where $\mathrm{k}_{1}$ and $\mathrm{k}_{2}$ are the release constants and $\mathrm{m}$ is the pure Fickian diffusion exponent. The exponent $\mathrm{m}$ is related to the aspect ratio of the tablets. . For the aspect ratio of tablet around 3 , the $m$ value is 0.45 . And $m=0.45$ was used in our study. The first term in the right hand of the Equation 4-3 represents the contribution of Fickian diffusion (F) and the second term represents the contribution of tablet structure relaxation $(R) . R / F$ ratio can be calculated using Equation 4-4:

$$
\mathrm{R} / \mathrm{F}=\mathrm{k}_{2} * \mathrm{t}^{\mathrm{m}} / \mathrm{k}_{1}
$$

The dominant drug release mechanism can be determined by the $\mathrm{R} / \mathrm{F}$ ratio at any given time during dissolution. The model fit was performed by the software GraphPad Prism 4.

\subsection{Results and Discussion}

\subsubsection{The effect of different pore formers on drug dissolution}

Incompletely release is a common problem associated with the hydrophobic matrix tablet. The typical solution is to add water soluble pore formers such as lactose and PEG to the matrix. The water soluble ingredient in the matrix will dissolve during dissolution and the pores will be formed in the matrix after the soluble material leached out. Those pores can act as the channels for water penetration and drug diffusion, therefore help to increase the drug release at the final stage. In our study, we selected three different pore formers. There are lactose, Starch 1500 and Polyox ${ }^{\circledR}$ 303. Lactose is a traditional pore former and easily dissolves in the water. Starch 1500 is a partially gelatinized starch and it is not soluble in the water. But it will expand after absorbing the dissolution medium thus pores or cracks will be created in tablet structure. Polyox ${ }^{\circledR} 303$ is a water soluble polymer. It has swelling property and can form a viscous gel upon contact with dissolution medium. The gel will be eroded during dissolution and leave empty space in the matrix for drug diffusion. The effect of the three pore formers on the drug release was shown in Figure 4-1. Compared to the formulation without any pore former $(\mathrm{H} 0)$, all the pore formers used in our study significantly increased the drug release at 12 hours $(\mathrm{P}<0.05)$. Due to the swelling property of $\operatorname{Starch} \AA 1500$ and Polyox ${ }^{\circledR}$ 303 , the swollen tablets with large amount of water filled pores and cracks were observed after dissolution for both formulations $\mathrm{P} 2$ and $\mathrm{H} 2$. The complete drug release $(>90 \%)$ at 12 hours was also observed for those two formulations. For formulation P1 which contains lactose as pore former, the external tablet size remained the same as original during dissolution. The drug release at 12 hours for P1 was only $78.59 \pm 0.40 \%$. This indicates the pores generated inside are not sufficient for complete drug release. The impact of the pore formers on drug release was exhibited not only on the final stage but also on the initial stage. A significant burst release at the first hour was observed for both 


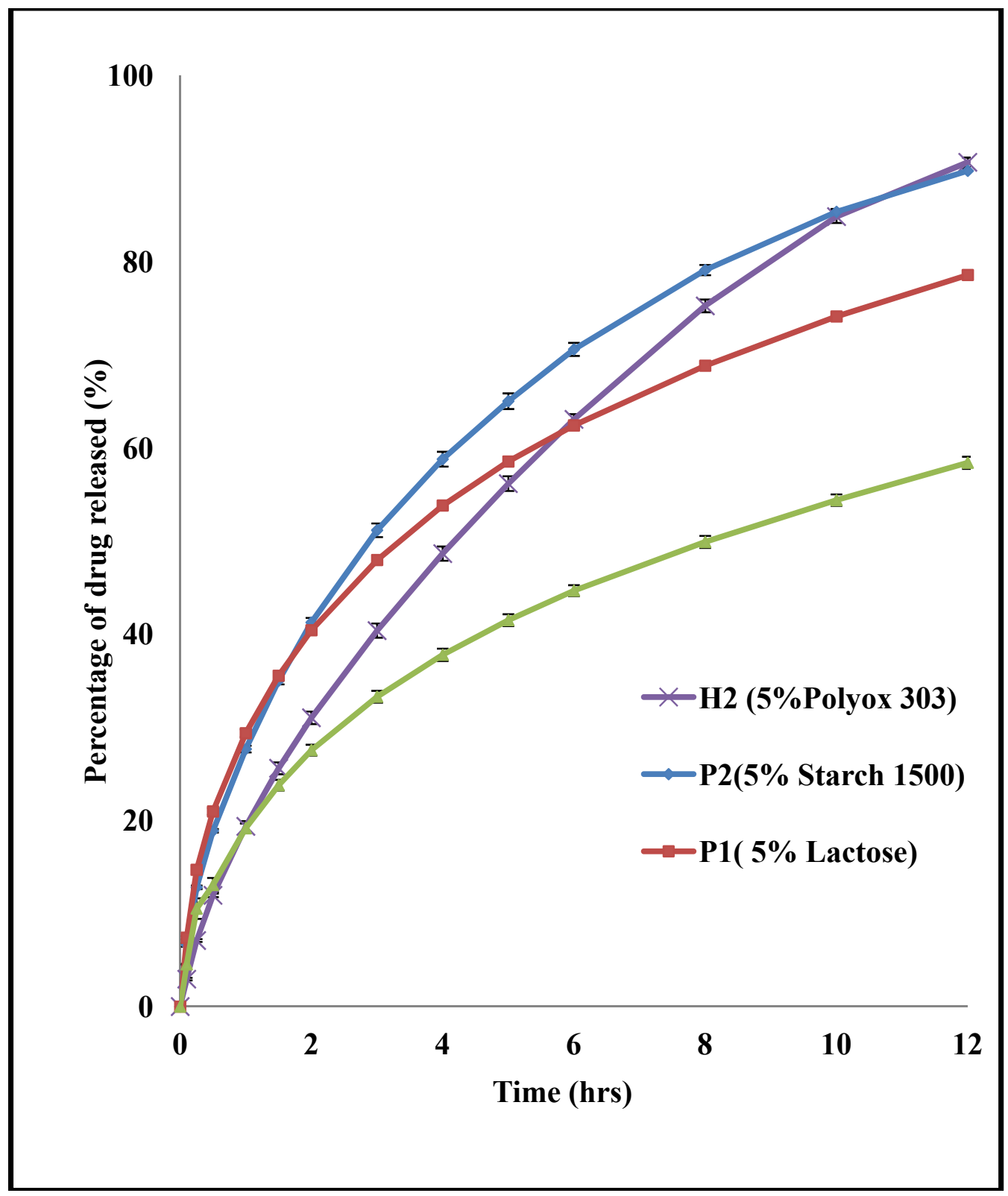

Figure 4-1. The effect of the pore formers on drug dissolution 
formulation P1 and P2. This can be explained by the hydrophilic nature of lactose and Starch 1500. They can facilitate the surface erosion of the tablet resulting rapid surface drug release. Formulation $\mathrm{H} 2$ which contains Polyox ${ }^{\circledR} 303$ as the pore former showed limited burst release at first hour. The first hour release of formulation $\mathrm{H} 2$ is similar as the formulation $\mathrm{H} 0$ without any pore former. Even though Polyox ${ }^{\circledR} 303$ also has a hydrophilic nature, the initial viscous gel formation helped to prevent the rapid burst release. The viscous gel formation counterbalanced the hydrophilic nature of Polyox ${ }^{\circledR}$ 303 Therefore a similar release rate during the first hour for formulation $\mathrm{H} 0$ and $\mathrm{H} 2$ was observed.

\subsubsection{The effect of the concentration of hydrophilic polymer on drug dissolution}

The concentration effect of the hydrophilic polymer was illustrated in Figure 4-2. Compared to the formulation without any hydrophilic polymer, addition of the hydrophilic polymer significantly increased the drug release at 12 hours $(\mathrm{P}<0.05)$. The addition of hydrophilic polymer has less influence on the initial drug release. The decreased drug release rate only took place on 0.25 hour while 0.5 hour and 1.0 hour remained similar $(\mathrm{P}>0.05)$. When the concentration of the hydrophilic polymer is in the range of $0-5 \%$, the drug release rate increased with increasing hydrophilic polymer concentration. Once the concentration of hydrophilic reached 5\%, further increasing the concentration of hydrophilic polymer to $10 \%$ did not change the drug release rate. This indicates the limit amount of the hydrophilic polymer that is required for improving the final drug release and an excess amount of hydrophilic polymer will not further contribute to modulating the drug release profile within these ranges.

\subsubsection{The type of hydrophilic polymer effect on drug dissolution}

Cellulose derivatives are most commonly used hydrophilic polymers in development of hydrophilic matrix tablets. To exclude the $\mathrm{pH}$ effect of the system, we selected three $\mathrm{pH}$ independent cellulose derivatives with similar viscosity but different substitution. These were HEC HHX, HPMC 100KM CR and HPC HXF. According to the chemical structure difference, the rank of hydrophilicity is HEC $>$ HPMC $>$ HPC. Due to the difference of the hydrophilicity, these polymers have different erosion and swelling behavior. It was demonstrated that those polymers exhibited different sustained release behavior as the hydrophilic matrix tablets were developed. In addition to the cellulose derivatives, another commonly used $\mathrm{pH}$ independent hydrophilic polymer polyethylene oxide (Polyox ${ }^{\circledR} 303$ ) was chosen. It is a hydrophilic polymer with relatively fast swelling and erosion rate. The type of hydrophilic polymer effect on the drug release was shown in Figure 4-3. Only formulation H5 prepared with the most hydrophobic polymer HPC showed slightly slower drug release at the final stage. The other dissolution curves are very close to each other. The $f_{2}$ value was used for comparison of these four dissolution profiles. We selected formulation $\mathrm{H} 2$ as reference, the $f_{2}$ values for $\mathrm{H} 4, \mathrm{H} 5$ and $\mathrm{H} 6$ are $64.09,69.65$ and 74.99 respectively. This result suggests the type of hydrophilic polymer has less impact on the drug release in this matrix system. 


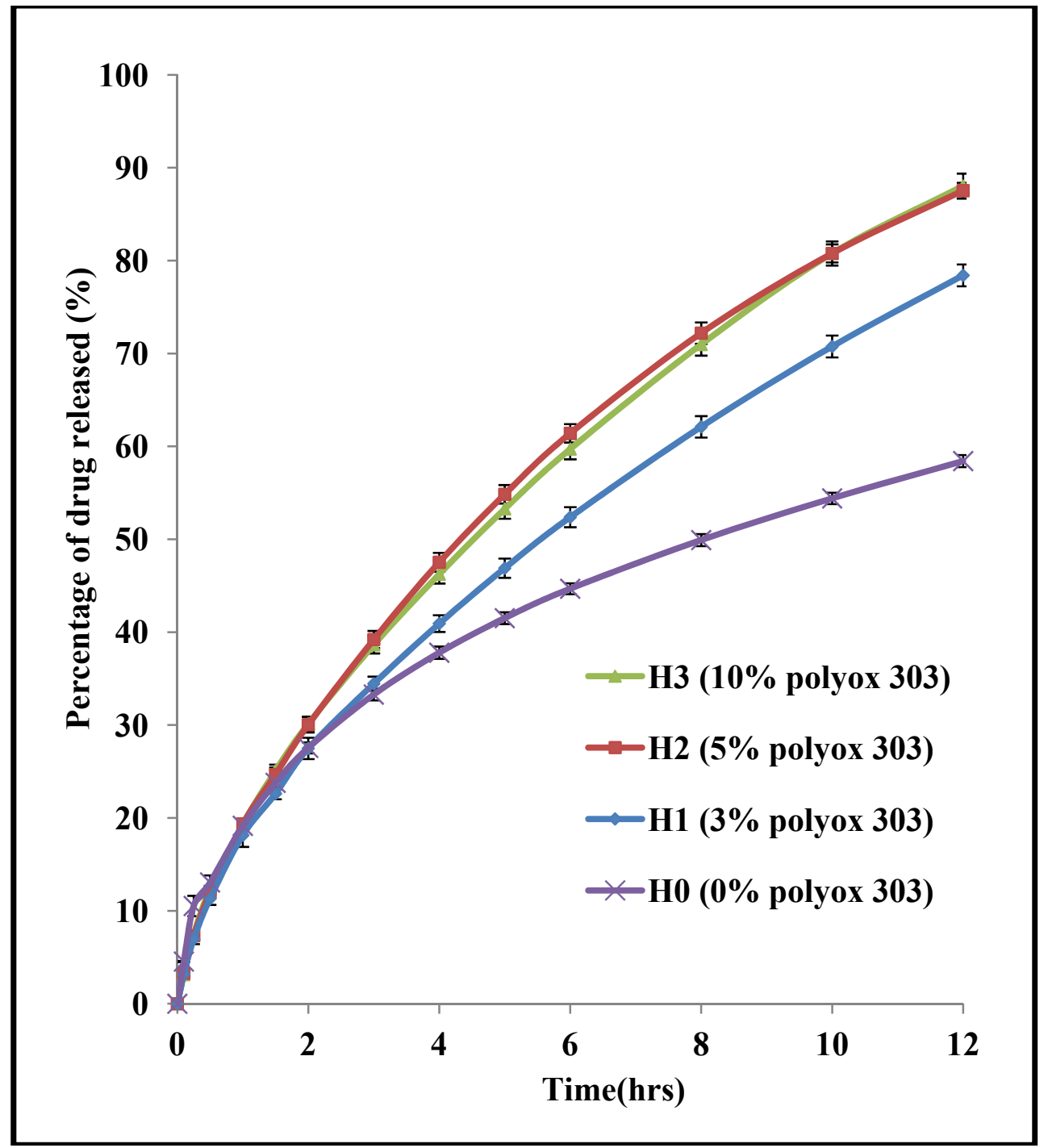

Figure 4-2. The effect of the concentration of hydrophilic polymer on drug dissolution 


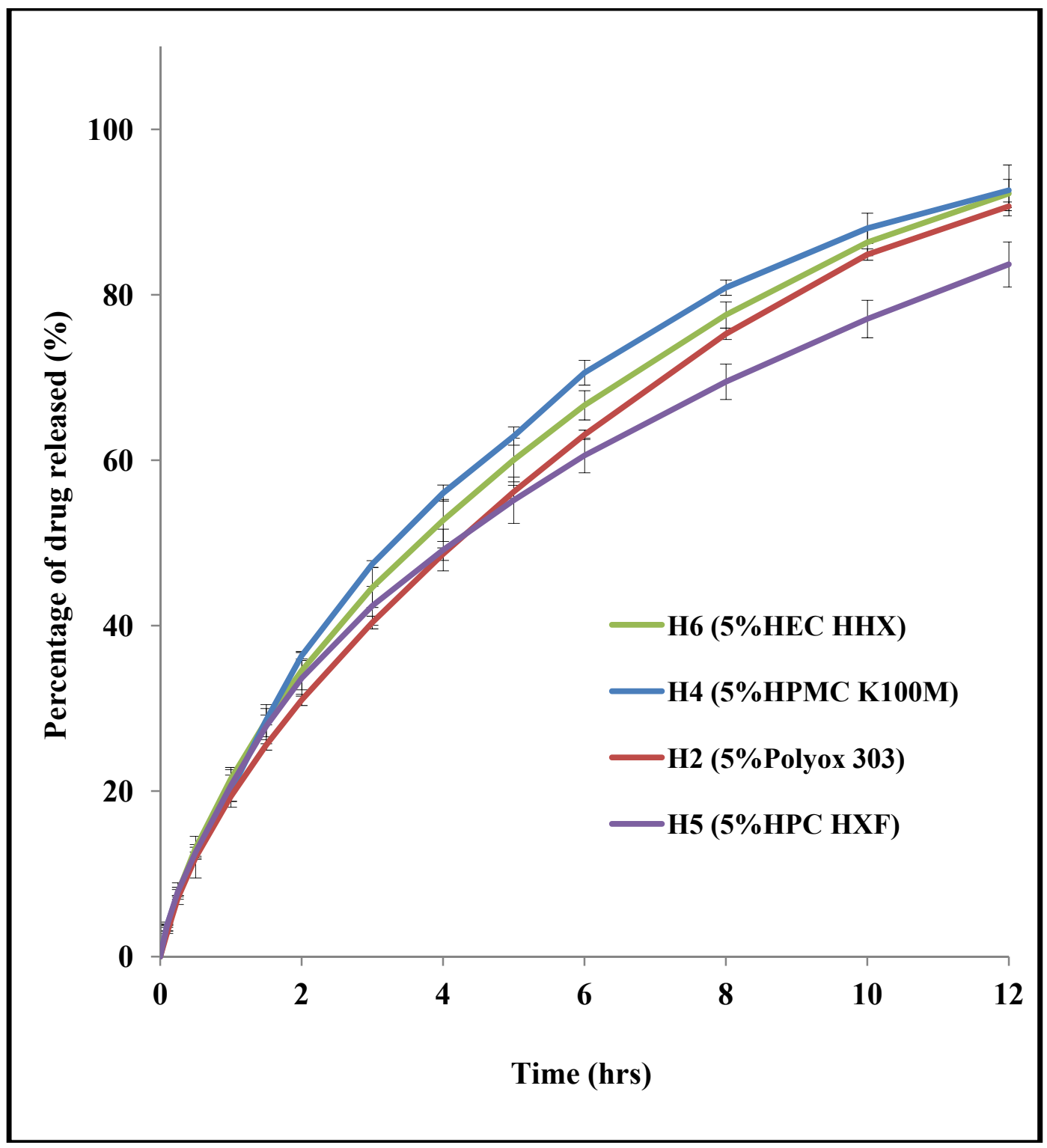

Figure 4-3. The effect of different hydrophilic polymers on drug dissolution 


\subsubsection{The effect of hydrophilic polymer viscosity grades on drug dissolution}

The viscosity grade of hydrophilic polymer is a major factor on controlling the drug release from the hydrophilic matrix system. In order to discover the effect of viscosity of hydrophilic polymer on drug release from the plastic inert matrix system, five different viscosity grades of HPMC were selected. The five grades of HPMC included two low viscosity grades E5 and E50, one middle viscosity grade $100 \mathrm{LV}$, two high viscosity grades $\mathrm{K} 4 \mathrm{M}$ and $\mathrm{K} 100 \mathrm{M}$. For the hydrophilic polymer with low viscosity, it could not be used alone for prepare the hydrophilic matrix tablets due to the less strength of gel layer and rapid erosion of tablets [2]. The result of polymer viscosity effect was demonstrated in Figure 4-4. Initially, the drug release rate slightly decreased with an increase in viscosity of hydrophilic polymer. The drug release decrease at the first hour is not statistically significant between formulations containing E5 and E50 (P>0.05), but statistically significant between formulations containing E5 and K100, K4M or K100M $(\mathrm{P}<0.05)$. There is no statistical difference on drug release at the first hour among the formulations containing $\mathrm{K} 100 \mathrm{LV}, \mathrm{K} 4 \mathrm{M}$ and $\mathrm{K} 100 \mathrm{M}$. At the final stage, the viscosity of the polymer effect is opposite to the initial stage. Drug release increased as an increase in the viscosity of the hydrophilic polymer. The significant drug release increase $(\mathrm{P}<0.05)$ at 12 hours was observed while increase the polymer viscosity from E5 to K100 LV. Further increase the polymer viscosity from K100 LV to K100M, the increase in drug release at 12 hours was not significant $(\mathrm{P}>0.05)$. This result can be explained by the insufficient swellability and less gel strength of the low viscosity grades of HPMC. Low viscosity grades HPMC formed weak gel layer initially then result rapid surface release and. insufficient swellability of low viscosity grades HPMC could not generate large size of pores for water penetration and drug diffusion then result incomplete release finally. From Figure 4-4, we also found that the middle to high viscosity grades polymer behave similar in terms of modulating the drug release from the plastic matrix system. We took formulation prepared with $\mathrm{K} 100 \mathrm{M}$ as a reference, $f_{2}$ values for formulations prepared with $\mathrm{K} 4 \mathrm{M}$ and $\mathrm{K} 100$ are 79.78 and 86.43 respectively. This may be due to two reasons: 1) the swellability of the hydrophilic polymer with higher viscosity was restricted by the hydrophobic matrix network; 2) the fast erosion rate of the middle viscosity grade hydrophilic polymer result drug leaches out from the matrix.

\subsubsection{The particle size of hydrophilic polymer effect on drug dissolution}

The particle size of the hydrophilic polymer is another factor which affects the drug release from the hydrophilic matrix system. The hydrophilic polymer with large particle size often exhibits slower hydration rate and result fast drug release. Three fractions of Polyox ${ }^{\circledR} 303$ were selected to study the particle size effect on the drug release from this matrix system. The result was shown in Figure 4-5. Three dissolution profiles are almost overlapped in this picture. This suggests the particle size of this hydrophilic polymer did not affect the drug release from this matrix system. This also indicates the drug release was not sensitive to the hydration rate of the hydrophilic polymer. 


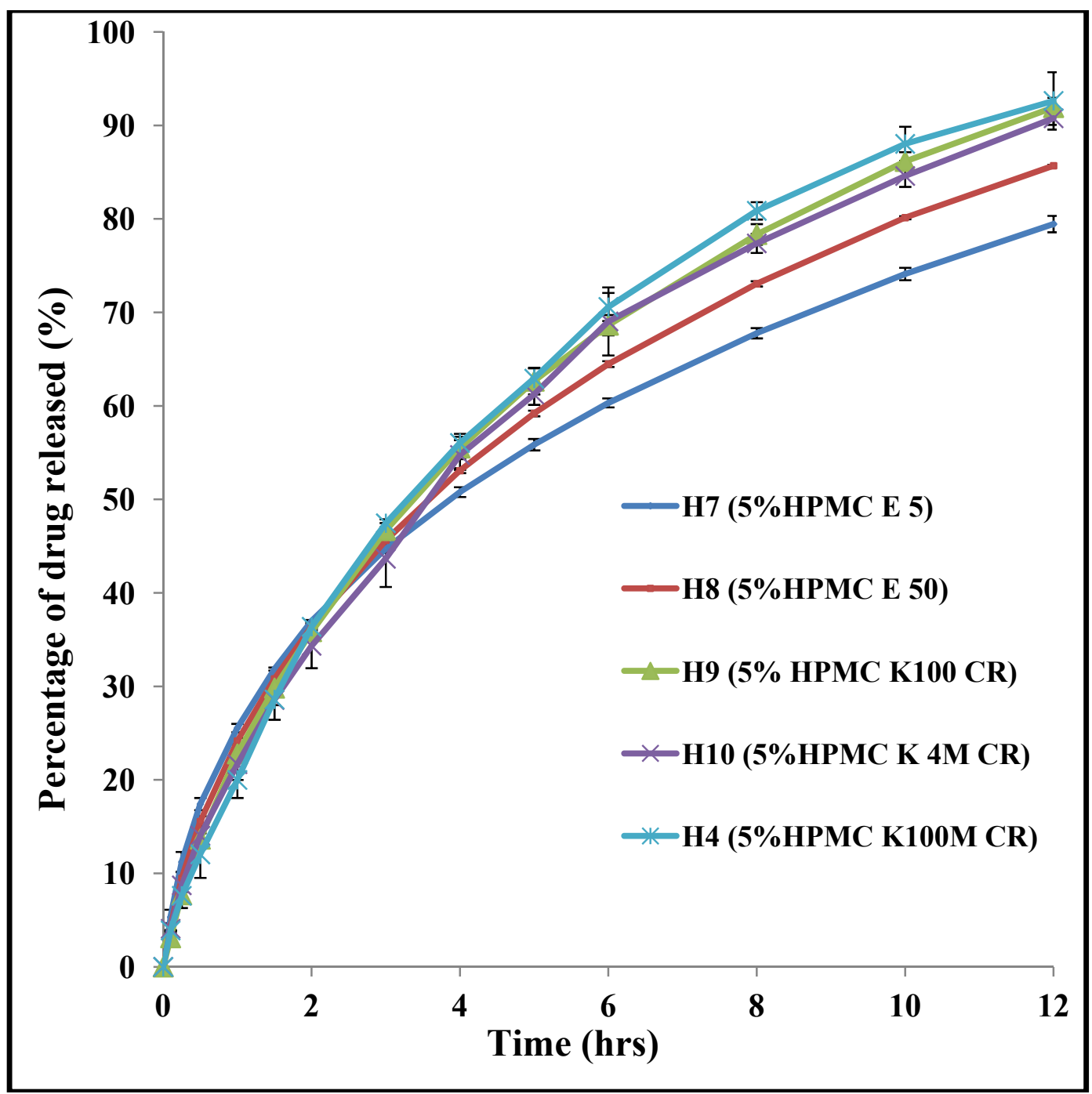

Figure 4-4. The effect of different viscosity of hydrophilic polymer on drug dissolution 


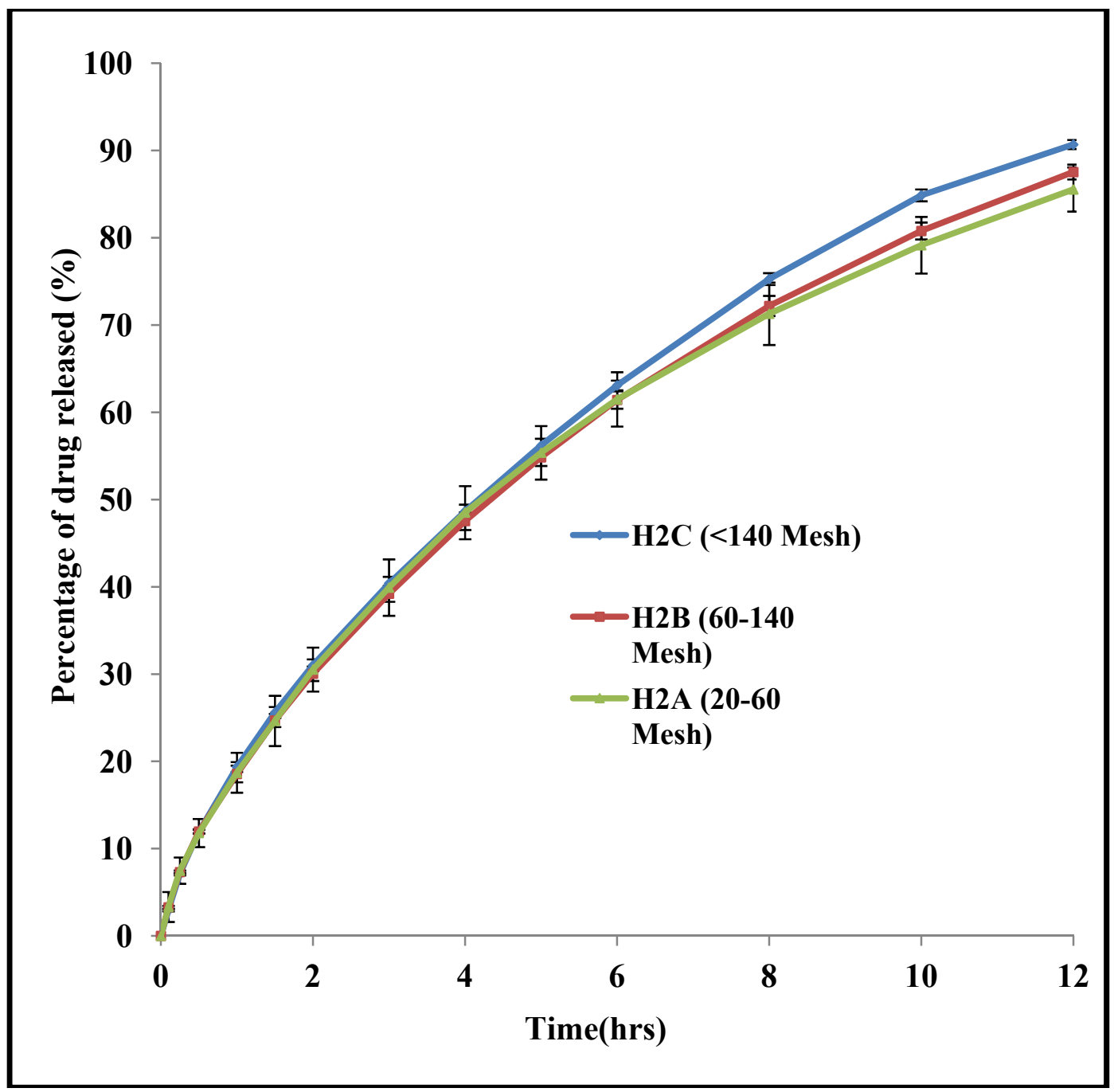

Figure 4-5. The effect of particle size of hydrophilic polymer on drug dissolution 


\subsubsection{The effect of formulation factors of co-processed excipient on drug dissolution}

Three different co-processed excipients prepared by spray dried method were selected. The hydrophilic polymer Polyox ${ }^{\circledR} 303$ was fixed at $5 \%$ for all formulations in the matrix. Figure 4-6 showed the effect of the co-processed excipient on drug dissolution. The tablets prepared by the unplasticized co-processed excipient F0 showed rapid drug release, more than $80 \%$ drug released at 6 hours. These tablets could not maintain their shape during the dissolution. All the tablets fall apart after dissolution. The unplasticized matrix could not withstand the stretch of the tablet. The rigid and brittle bond was broken by the swell of the hydrophilic polymer. On the contrast, the plasticized matrix tablets $\mathrm{H} 2$ (ATBC as plasticizer) and H12 (ATEC as plasticizer) all swollen but still kept intact at the end of dissolution. This can be explained by the plasticizer effect. The plasticizer contributes to the flexibility and elongation of the tablet matrix therefore help to maintain the integrity of the tablet. As shown in Figure 4-6, the tablets prepared with co-processed excipient F1 (ATEC as plasticizer) showed similar dissolution profile as the tablets prepared with co-processed excipient F2 (ATBC as plasticizer). The similarity factor $\mathrm{f} 2$ value of these two dissolution profile is 77.78 . This indicates the type of the plasticizer in co-processed excipient has less impact on drug release from this matrix system.

\subsubsection{The effect of pH of dissolution medium on drug dissolution}

The model drug propranolol hydrochloride is a hydrochloride salt with pka 9.5. When the $\mathrm{pH}$ ranges from 1.2 to 7.4 , it shows a $\mathrm{pH}$ independent solubility profile. The hydrophilic polymer Polyox ${ }^{\circledR} 303$ is a nonionic polymer and its solubility is not sensitive to $\mathrm{pH}$. The result of $\mathrm{pH}$ effect on drug dissolution was shown in Figure 4-7. The drug release profiles of $\mathrm{H} 2$ in three different media are very close to each other. Select water release as reference, the $f_{2}$ value of the simulated gastric fluid (SGF) is 67.68 and the simulated intestinal fluid (SIF) is 76.99. The result suggests this novel matrix system provides a $\mathrm{pH}$ independent dissolution profile for a $\mathrm{pH}$ insensitive drug. But for a $\mathrm{pH}$ sensitive drug, the dissolution profile may change based on the solubility of drug in different $\mathrm{pH}$ medium.

\subsubsection{Drug release mechanism study}

\subsubsection{Optical microscopic observation}

Figures 4-8 and 4-9 recorded the water penetration and components changes of two colored tablet formulations of $\mathrm{H} 0$ and $\mathrm{H} 2$ during 12 hours dissolution. Formulation $\mathrm{H} 0$ does not contain any hydrophilic polymer. The white drug powder was uniformly distributed in the matrix before dissolution as described in Figure 4-8A and B. Upon contacting with water, the surface drug dissolved in the water and left the pores on the surface of the tablet (Figure 4-8B) and the pore size is as same as the size of undissolved 


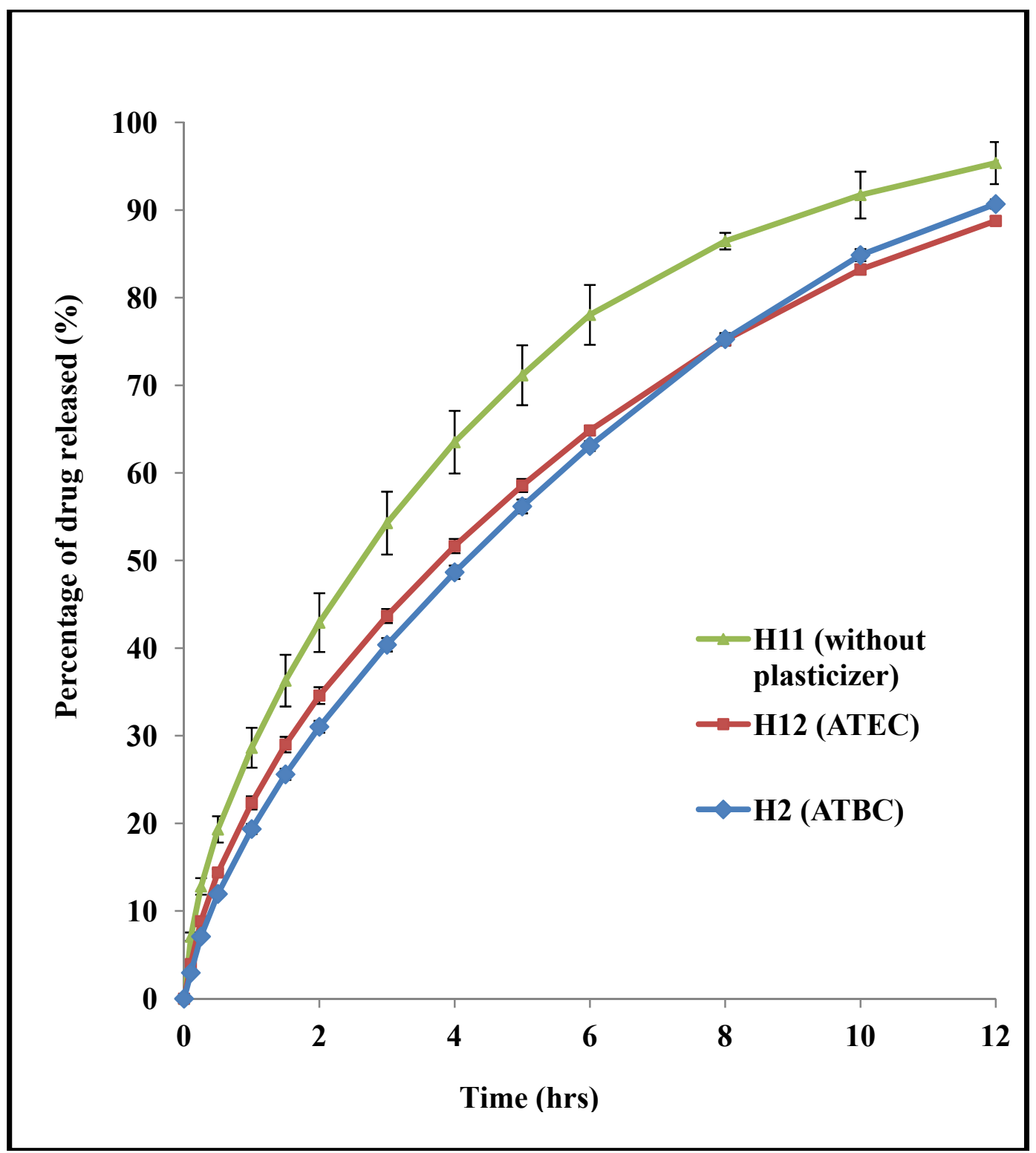

Figure 4-6. The effect of co-processed excipients on drug dissolution 


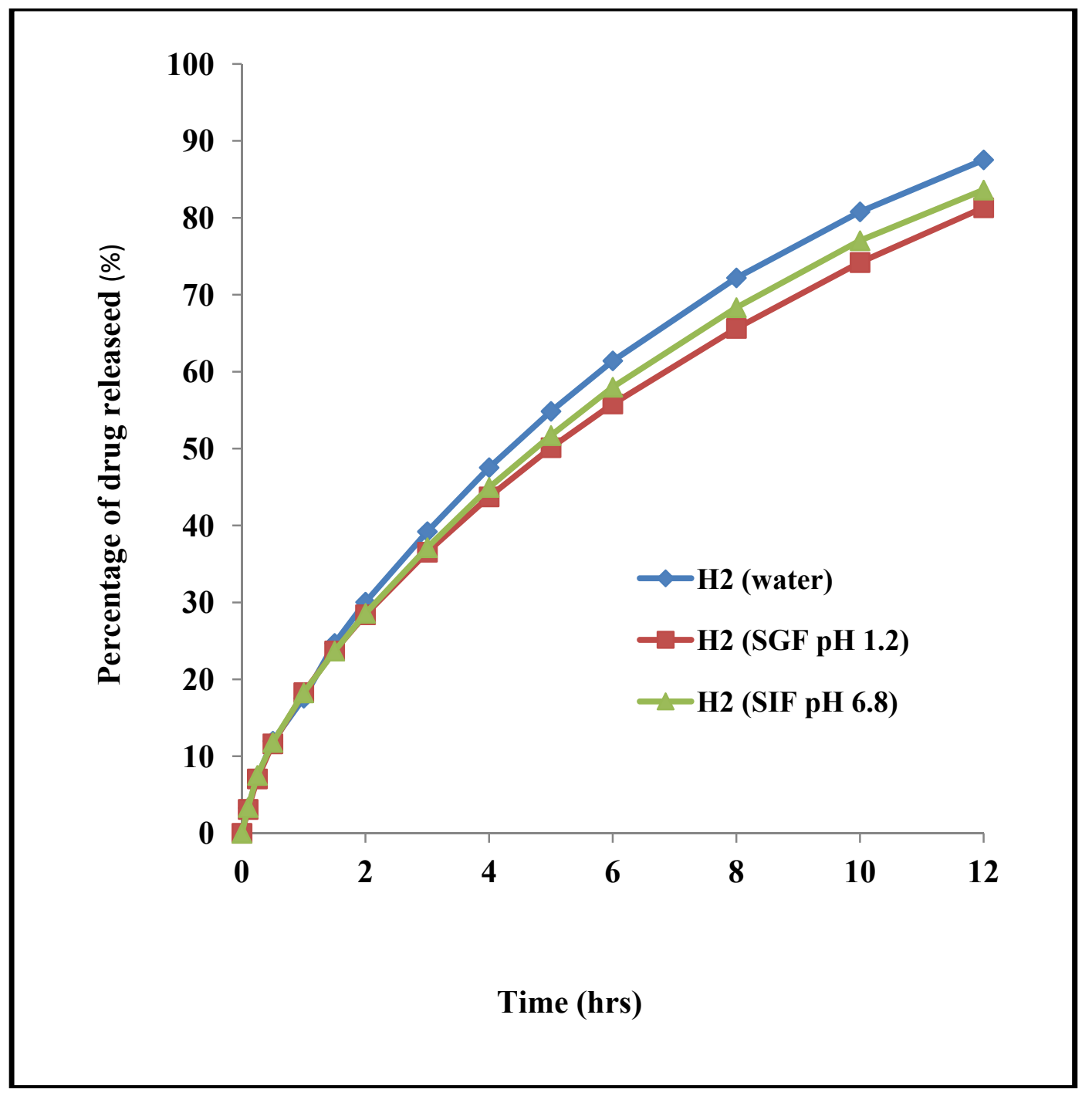

Figure 4-7. pH effect on drug dissolution 

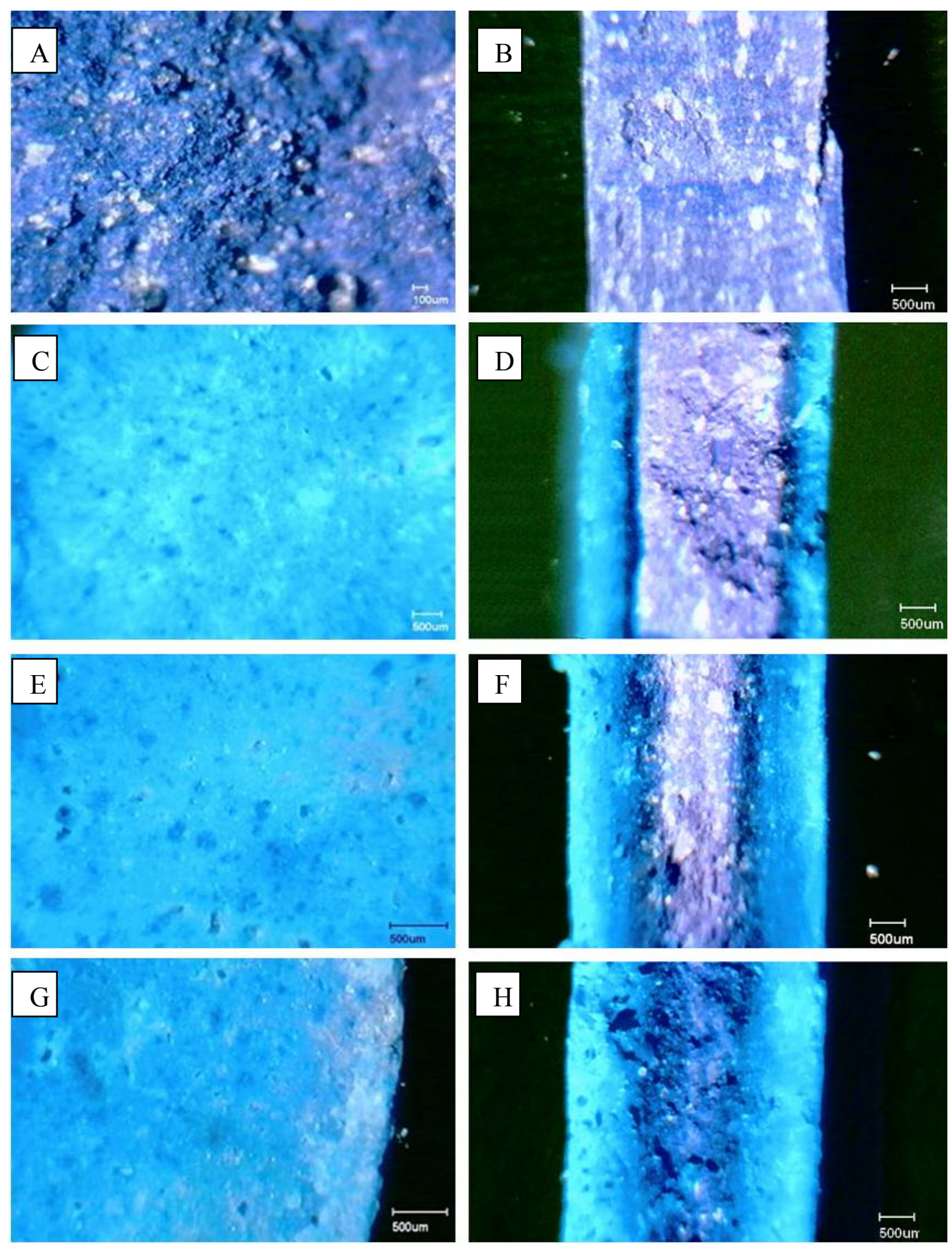

Figure 4-8. Microscopic picture of colored tablets (H0) during dissolution A: Tablet surface at 0 hour; B: Tablet cross section at 0 hour; $C$ : tablet surface at 1 hour; D: tablet cross section at 1 hour; E: Tablet surface at 6 hours; F: tablet cross section at 6 hours; G: tablet surface at 12 hours; F: tablet cross section at 12 hours. 

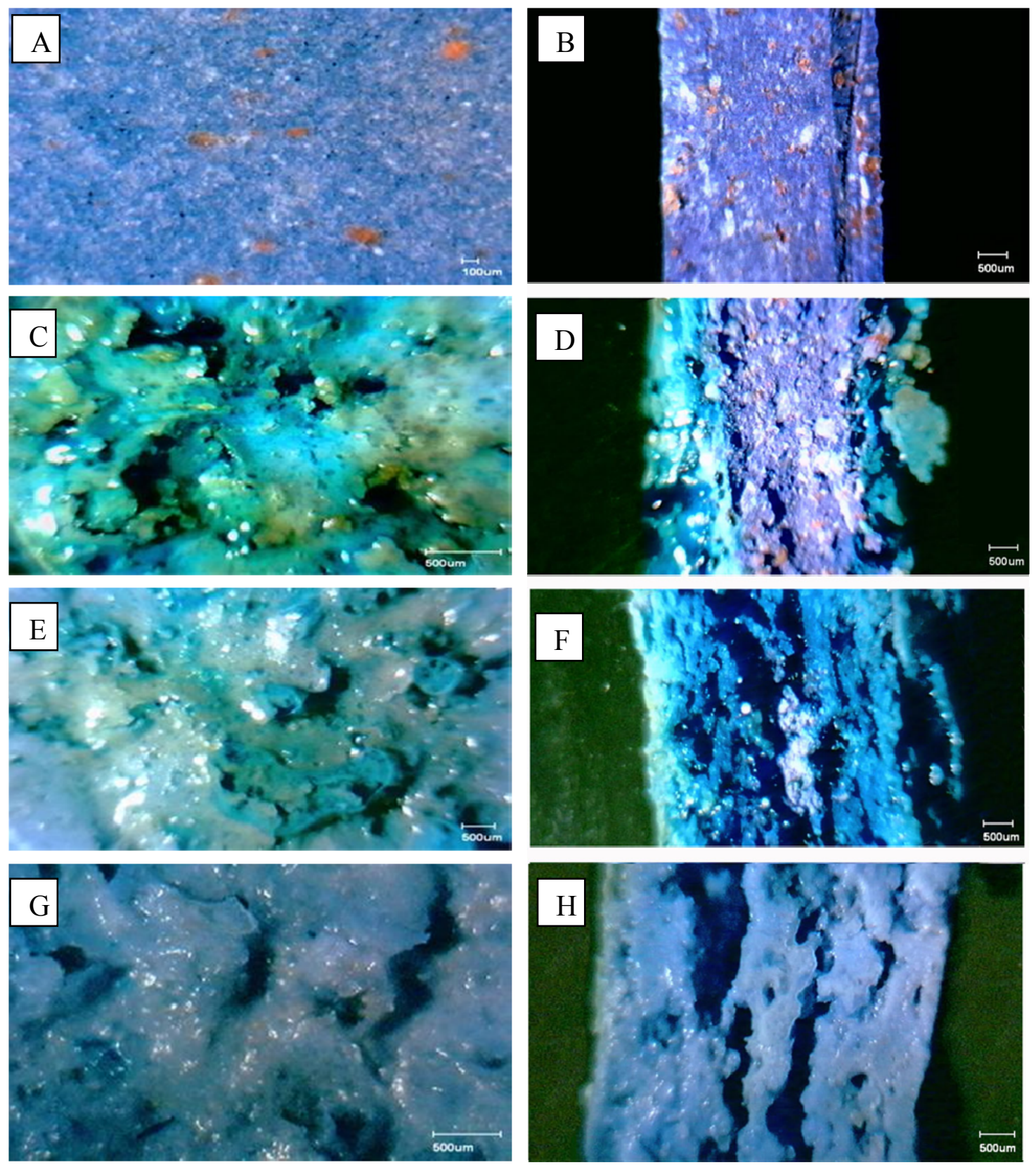

Figure 4-9. Microscopic picture of colored tablets (H2) during dissolution

A: Tablet surface at 0 hour; B: tablet cross section at 0 hour; $C$ : tablet surface at 1 hour; D: tablet cross section at 1 hour; E: tablet surface at 6 hours; F: tablet cross section at 6 hours; G: tablet surface at 12 hours; F: tablet cross section at 12 hours. 
drug. Figure 4-8D, $\mathbf{E}$ and $\mathbf{F}$ demonstrated the slow water penetration process through the pores into the core of the tablet. As shown in Figure 4-8H, even water completely wet the tablet at 12 hours, there are still around $40 \%$ drug remaining in the center of the tablet and could not diffuse through the tightly matrix structure. As described in Table 4-7, the geometric dimension of $\mathrm{H0}$ kept constant during 12 hours dissolution. The slower water penetration rate and the compacted tablet structure result incomplete release.

Formulation $\mathrm{H} 2$ contains 5\% Polyox 303 and the color of Polyox 303 is orange in the Figure 4-8. Initially, the hydrophilic polymer, drug and the co-processed excipient were uniformly distributed in the matrix (Figure 4-9A and B). After $1 \mathrm{~h}$, the hydrophilic polymer located on the surface was swollen and formed a viscous gel. The large pores filled with viscous gel on the tablet surface were observed (Figure 4-9C). This gel can prevent the rapid surface drug release initially. With the swelling of the hydrophilic polymer, the tablet dimensions changed both radically and axially at 1 hour (Figure 4-9D and Table 4-7). At 6 hours, the hydrophilic polymer located on the surface dissolved and left the swollen pores on the surface for water penetration. The tablets continued to expand and the water almost wetted the whole tablet at 6 hours. Due to the present of large pores, water penetration rate for formulation $\mathrm{H} 2$ is much faster than formulation $\mathrm{H} 0$. The geometric dimension of the tablet at 12 hours was similar to 6 hours as shown in Table 4-7. This indicates the hydrophilic polymer was completely hydrated at 6 hours. After 6 hours, the polymer erosion is the major activity of the hydrophilic polymer within the tablet matrix. At 12 hours, highly porous structure of the tablet can be observed in Figure 4-9G and 9H. Because the large pores generated while the dissolution, water can easily penetrate through these large pores and extract drug from the matrix. Therefore completely drug release can be expected from formulation $\mathrm{H} 2$.

\subsubsection{Mathematic model fit}

The dissolution profiles of three formulations containing different pore formers were fitted to logarithm transformed power law equation and Peppas and Shalin equation. The results were shown in Figures 4-10 and 4-11. All the three dissolution profiles displayed a good fit to both the power law equation and Peppas and Shalin equation $\left(\mathrm{R}^{2}>0.98\right)$. The drug release mechanism can be determined by the $\mathrm{n}$ value which is the slope of logarithm transformed power law equation and $\mathrm{R} / \mathrm{F}$ ratio which can be calculated from the Peppas and Shalin equation. The $\mathrm{n}$ value for $\mathrm{P} 1$ (lactose as pore former) is slightly higher than 0.45 , this suggest the major drug release is controlled by Fickian diffusion. This is confirmed by the Peppas and Shalin equation. The $\mathrm{k}_{2}$ value for $\mathrm{P} 1$ in Peppas and Shalin equation is negative. The negative value of $\mathrm{k}_{2}$ means the structure relaxation can be neglected. The $\mathrm{n}$ value $\mathrm{P} 2$ formulation (Starch 1500 as pore former) in power law equation is 0.5703 . This value is higher than 0.45 and indicates both Fickian diffusion and polymer relaxation play a role in controlling the drug release. The $\mathrm{k}_{2} / \mathrm{k}_{1}$ ratio is 0.206 and the $\mathrm{R} / \mathrm{F}$ ratio for $\mathrm{P} 2$ formulation is always less than 1 within 12 hours dissolution time. This indicates the Fickian diffusion is dominant mechanism for P2 tablets. For the $\mathrm{H} 2$ formulation (Polyox 303 as pore former), the $\mathrm{n}$ value is 0.6781 . It is also an anomalous transport mechanism. Both diffusion and polymer swelling take place. 
Table 4-7. Tablets size change during dissolution

\begin{tabular}{ccccc}
\hline \multirow{2}{*}{ Time } & \multicolumn{2}{c}{ H0 } & \multicolumn{2}{c}{ H2 } \\
\cline { 2 - 5 } & $\begin{array}{c}\text { Thickness } \\
(\mathbf{m m})\end{array}$ & $\begin{array}{c}\text { Diameter } \\
\mathbf{( m m )}\end{array}$ & $\begin{array}{c}\text { Thickness } \\
(\mathbf{m m})\end{array}$ & $\begin{array}{c}\text { Diameter } \\
\mathbf{( m m})\end{array}$ \\
\hline 0 hour & 3.68 & 9.55 & 3.70 & 9.55 \\
1 hour & 3.70 & 9.60 & 4.55 & 9.81 \\
6 hours & 3.70 & 9.60 & 6.07 & 9.85 \\
12 hours & 3.70 & 9.60 & 6.11 & 9.84 \\
\hline
\end{tabular}




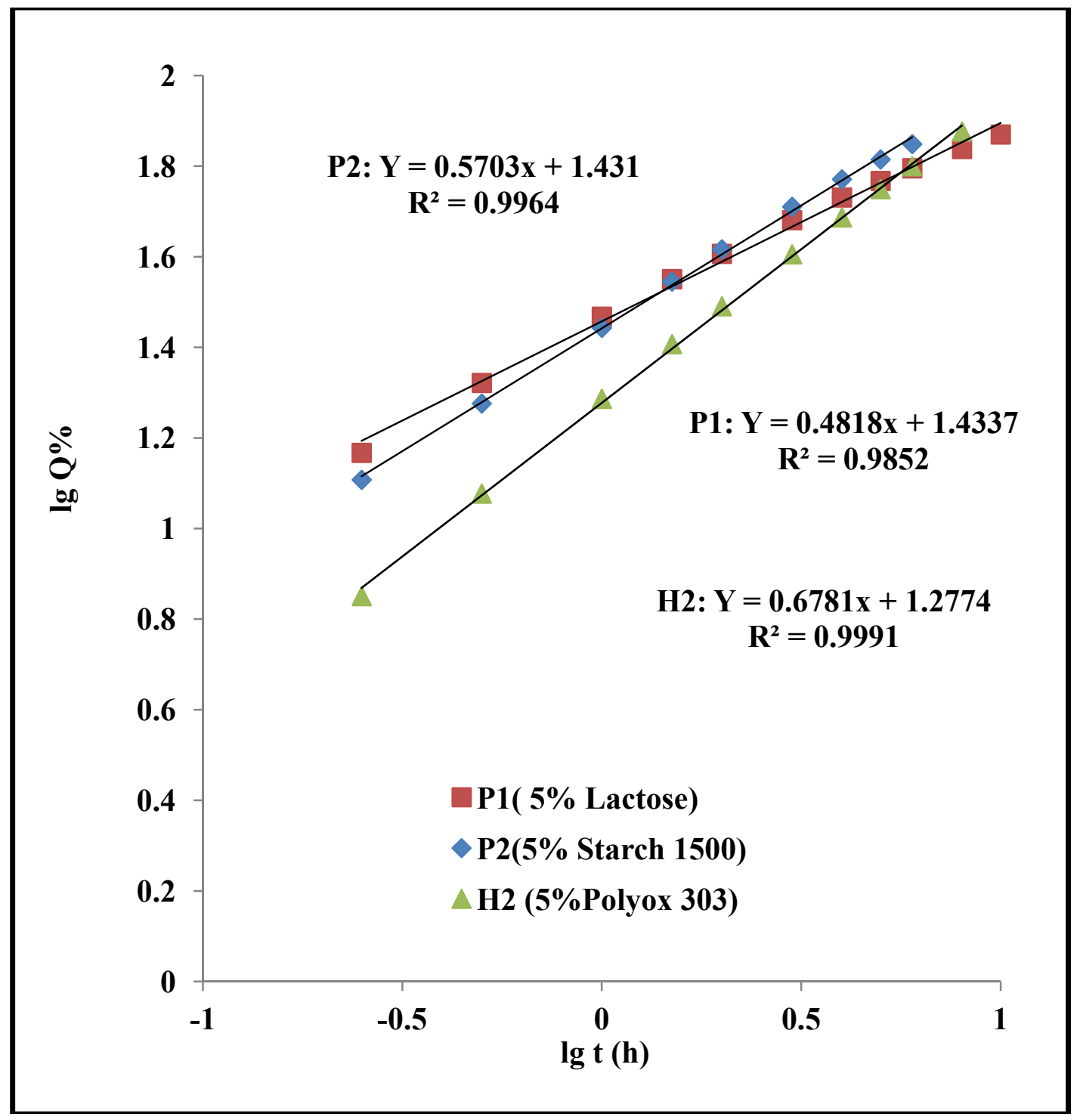

Figure 4-10. Power law equation fit 


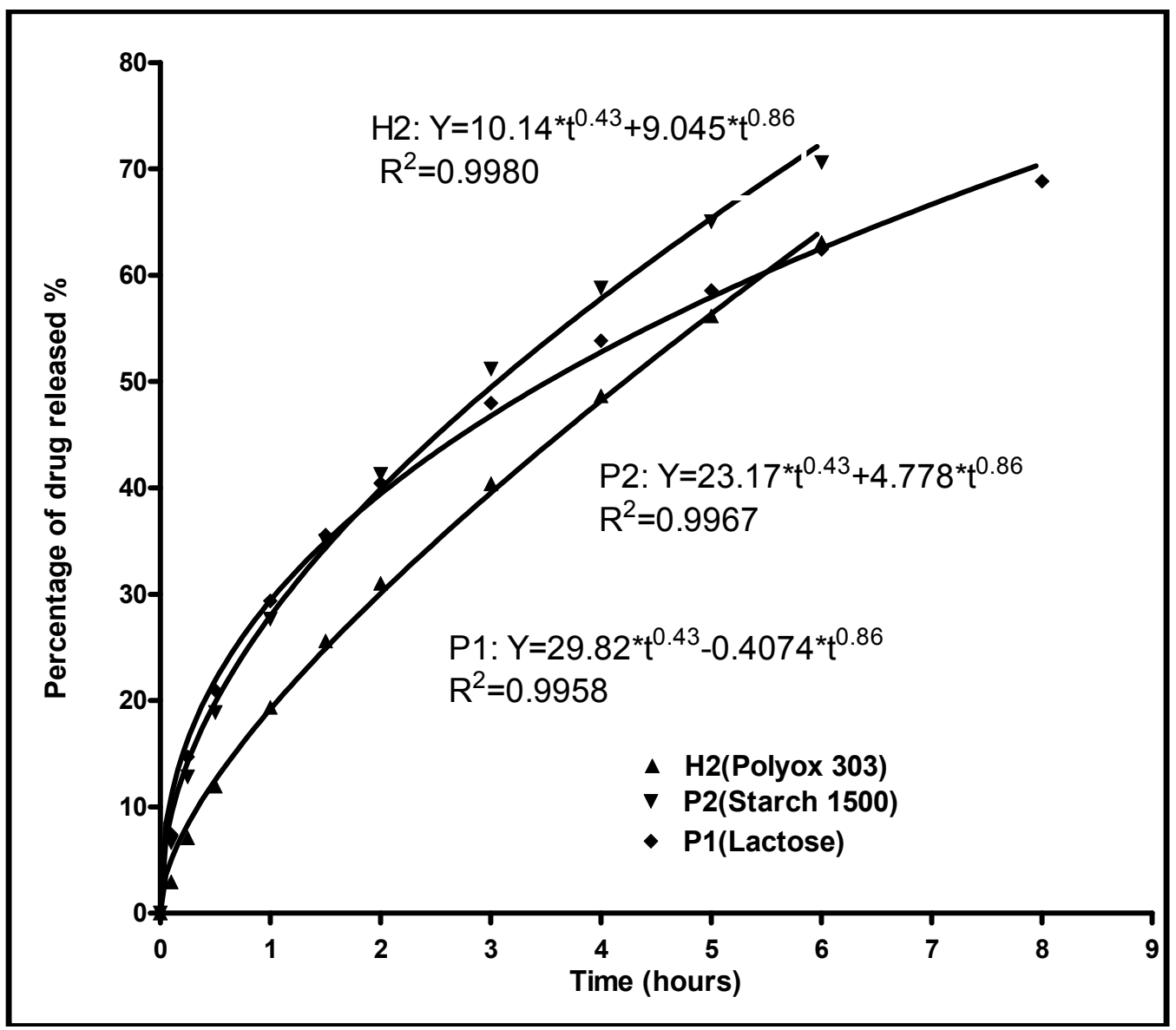

Figure 4-11. Peppa and Shalin equation fit 
The $\mathrm{k} 2 / \mathrm{k} 1$ ratio for $\mathrm{H} 2$ is 0.892 . When $\mathrm{t}<1.3$ hours, $\mathrm{R} / \mathrm{F}$ is less than 1 . The Fickian diffusion is the dominant release mechanism. This is diffusion is caused by the release of the surface drug. When $t>1.3$ hours, the matrix relaxation is the dominant release mechanism. This is caused by the polymer swelling and erosion.

\subsection{Conclusions}

A novel swellable porous matrix tablet was successfully developed by incorporation of small amounts of hydrophilic polymer into the plastic inert matrix. The drug release from this novel matrix formulation showed a limited burst release initially with complete release at final stage.

The drug release rate from this matrix system is independent with the type and the particle size of hydrophilic polymer and the $\mathrm{pH}$ of dissolution medium. The drug release rate increased when the hydrophilic polymer concentration less than $5 \%$. Further increase the hydrophilic polymer concentration to $10 \%$ did not further increase the drug release rate. The low viscosity grade hydrophilic polymer result rapid burst release and incomplete final release, while middle to high viscosity grades of hydrophilic polymer avoided these problems. The optical microscopy results revealed the initial gel formation on the tablet surface with development of a swollen porous structure in the matrix network during dissolution. The drug release is controlled by both drug diffusion and polymer relaxation. The Fickian diffusion is the dominant release mechanism at initial stage and the polymer relaxation becomes dominant thereafter. 


\section{Chapter 5. Application of the Novel Matrix System on the Drugs with Different Solubility}

\subsection{Introduction}

The final objective of this work is to apply the plasticized cellulose acetate excipient and the hydrophilic polymer involved novel matrix system to the sustained release matrix tablets. In order to test the suitability of this swollen porous matrix system, we selected three different drugs with different solubility as model drugs. A $\beta$-adrenergic blocking agent propranolol hydrochloride with water solubility more than $150 \mathrm{mg} / \mathrm{ml}$ at $37^{\circ} \mathrm{C}$ was selected as the freely water soluble model drug [83]. Theophylline which used for treatment of chronic asthma with water solubility of $9.9 \mathrm{mg} / \mathrm{ml}$ at $37^{\circ} \mathrm{C}$ was selected as sparingly water soluble model drug [36]. The short acting sulphonyl urea drug glipizide with solubility of $0.078 \mathrm{mg} / \mathrm{ml}$ in $\mathrm{pH} 6.8$ and insoluble in water and acidic $\mathrm{pH}$ was selected as poorly water soluble model drug $[23,84]$. Propranolol hydrochloride and glipizide all have a short elimination half-life and theophylline has a fairly narrow therapeutic plasma concentration. The clinical effect of those drugs can be improved by using sustained release formulations. The feasibility of the co-processed excipient and the novel matrix system in these three drugs are evaluated by comparison of dissolution profiles with USP specifications or commercial product.

\subsection{Materials and Methods}

\subsubsection{Materials}

Cellulose acetate with $39.8 \%$ acetyl content and $38 \mathrm{cp}$ viscosity (CA 398-10 NF, Eastman, Kingsport, TN) was used as the main polymer in this study. Triethyl citrate (TEC), acetyltriethyl citrate (ATEC), acetyltributyl citrate (ATBC) and dibutyl sebacate (DBS) (Morflex Inc., Greensboro, NC) were used as plasticizers. Milled dicalcium phosphate dihydrate (milled DCP Innophos Inc., Cranbury, IL) were used as filler. Magnesium stearate (Mallinckrodt, Paris, KY) was used as a lubricant. Propranolol hydrochloride was used as water soluble model drug, Theophylline anhydrous (BASF, Ludwigshafen, Germany) was used as sparing water soluble drug and glipizide was used as poorly water soluble drug. Three different viscosity grades of HPC(Klucel ${ }^{\circledR}$ EXF,GXF and HXF, Ashland, Wilmington, DE), high viscosity grade of polyethylene oxide (Polyox ${ }^{\circledR} 303$, Dow Chemical, Midland MI) and high viscosity grade of sodium alginate (KELTONE® HVCR, FMC biopolymer, Philadelphia, PA) were selected as hydrophilic polymers. Glucotrol XL ${ }^{\circledR} 10 \mathrm{mg}$ (Pfizer, Peapack, NJ) was used as a commercial product for dissolution profile comparison. 


\subsubsection{Methods}

\subsubsection{Tablets preparation}

\subsection{Propranolol hydrochloride tablets}

The tablet formulations were listed in Tables 5-1 and 5-2. The drug was mixed with intragranular hydrophilic polymer by passing through 20 mesh sieve three times. The mixture was compacted to form a ribbon on the TFC-LAB Micro Roller Compactor (Vector, Marion, IA). The settings for the roller compactor are: Roller speed: 2rpm, screw speed: $20 \mathrm{rpm}$, roller pressure: $5 \mathrm{MPa}$. The ribbons were milled by the minioscillator mounted with an 18 mesh sieve. The dry granules were mixed with other ingredients by passing through 16 mesh sieve for three times and then compressed on a Carver Press (Carver Inc., Wabash, IN) by $3 / 8$ inch flat face punch at 1.5 metric ton compression force with 3 second dwell time. The target weight was $400 \mathrm{mg}$.

\subsection{Theophylline anhydrous tablets}

The tablet formulations were listed in Table 5-3. The tablets were prepared by a direct compression method. All the ingredients were mixed by passing through 20 mesh sieve for 3 times. The mixture was compressed on a Carver Press (Carver Inc., Wabash, IN) by $3 / 8$ inch flat face punch at 1.5 metric ton compression force with 3 second dwell time. The target weight was $400 \mathrm{mg}$.

\subsection{Glipizide tablets}

The tablet formulations were listed in Tables 5-4 and 5-5. The tablets were prepared by a direct compression method. All the ingredients were mixed by passing through 20 mesh sieve for 3 times. The mixture was compressed on a Carver Press (Carver Inc., Wabash, IN) by 1/4 inch flat face punch at 1.0 metric ton compression force with 3 second dwell time. The target weight was $200 \mathrm{mg}$.

\subsubsection{In vitro dissolution study}

\subsection{Propranolol hydrochloride and theophylline}

The USP Apparatus II (Hanson SR-8 Plus, Hanson Research Corporation, Chatsworth, CA) was used to evaluate the drug release. Three tablets from each batch were tested. $900 \mathrm{ml}$ of distilled water and $\mathrm{pH} 7.4$ phosphate buffer at $37 \pm 0.5^{\circ} \mathrm{C}$ was used as the dissolution medium to evaluate the release of propranolol hydrochloride and 
Table 5-1. Formulations for propranolol hydrochloride tablets prepared with different intragranular hydrophilic polymers

\begin{tabular}{lccc}
\hline \multirow{2}{*}{ Ingredients } & \multicolumn{3}{c}{ Percentage (\%) } \\
\cline { 2 - 4 } & PR1 & PR2 & PR3 \\
\hline Propranolol hydrochloride & 20 & 20 & 20 \\
Polyox® 303(intragranular) & 5 & 0 & 0 \\
HPC HXF (intragranular) & 0 & 5 & 0 \\
HPC GXF (intragranular) & 0 & 0 & 5 \\
Spray dried excipient F2 & 74.5 & 74.5 & 74.5 \\
Magnesium Stearate & 0.5 & 0.5 & 0.5 \\
Total & 100 & 100 & 100 \\
\hline
\end{tabular}

Table 5-2. Formulations for propranolol hydrochloride tablets prepared with intragranular and extragranular hydrophilic polymers

\begin{tabular}{lcc}
\hline Ingredients & \multicolumn{2}{c}{ Percentage (\%) } \\
\cline { 2 - 3 } & PR4 & PR5 \\
\hline Propranolol hydrochloride & 20 & 20 \\
HPC GXF(intragranular) & 5 & 0 \\
HPC EXF (intragranular) & 0 & 5 \\
Polyox® 303(extragranular) & 5 & 5 \\
Spray dried excipient F2 & 69.5 & 69.5 \\
Magnesium Stearate & 0.5 & 0.5 \\
Total & 100 & 100 \\
\hline
\end{tabular}


Table 5-3. Formulations of theophylline tablets prepared with different concentrations of hydrophilic polymers

\begin{tabular}{lccc}
\hline \multirow{2}{*}{ Ingredients } & \multicolumn{3}{c}{ Percentage (\%) } \\
\cline { 2 - 4 } & T1 & T2 & T3 \\
\hline Propranolol hydrochloride & 25 & 25 & 25 \\
Spray dried excipient F2 & 74.5 & 69.5 & 64.5 \\
Polyox ${ }^{\circledR}$ 303 & 0 & 5 & 10 \\
Magnesium Stearate & 0.5 & 0.5 & 0.5 \\
Total & 100 & 100 & 100 \\
\hline
\end{tabular}

Table 5-4. Formulations of glipizide tablets with different concentrations of coprocessed excipients

\begin{tabular}{lcccc}
\hline \multirow{2}{*}{ Ingredients } & \multicolumn{4}{c}{ Percentage (\%) } \\
\cline { 2 - 5 } & G1 & G2 & G3 & G4 \\
\hline Glipizide & 5 & 5 & 5 & 5 \\
Spray dried excipient F2 & 5 & 7.5 & 10 & 20 \\
Lactose 316 & 89.5 & 87.0 & 84.5 & 74.5 \\
Magnesium Stearate & 0.5 & 0.5 & 0.5 & 0.5 \\
Total & 100 & 100 & 100 & 100 \\
\hline
\end{tabular}

Table 5-5. Formulations of glipizide tablets prepared with different concentrations of sodium alginate

\begin{tabular}{lccc}
\hline \multirow{2}{*}{ Ingredients } & \multicolumn{3}{c}{ Percentage (\%) } \\
\cline { 2 - 4 } & G5 & G6 & G7 \\
\hline Propranolol hydrochloride & 5 & 5 & 5 \\
Spray dried excipient F2 & 20 & 20 & 20 \\
Lactose 316 & $73.5 \%$ & $72.5 \%$ & $71.5 \%$ \\
Sodium Alginate HVCR & 1 & 2 & 3 \\
Magnesium Stearate & 0.5 & 0.5 & 0.5 \\
Total & 100 & 100 & 100 \\
\hline
\end{tabular}


theophylline respectively. The paddle speed was 50rpm. The samples were taken at predetermined time points for 12 hours. The absorbance of the solution was measured at $289 \mathrm{~nm}$ for propranolol hydrochloride and $274 \mathrm{~nm}$ for theophylline by a UVspectrophotometer (Perkin-Elmer Corporation, Norwalk, CT). The drug concentration and the percentage of drug release were calculated by the software DissLab $®$.

\subsection{Glipizide}

The USP Apparatus II (Hanson SR-8 Plus, Hanson Research Corporation, Chatsworth, CA) was used to evaluate the drug release. Three tablets from each batch were tested. Hydrochloric acid $(0.1 \mathrm{~N}, 750 \mathrm{ml})$ was used as the dissolution media for the first 2 hours. Then, $\mathrm{pH}$ was adjusted to 6.8 by adding $250 \mathrm{ml}$ of $1 \mathrm{~N}$ tribasic phosphate. The paddle speed is $50 \mathrm{rpm}$. The samples were taken at predetermined time points for 14 hours. The absorbance of the solution was measured at $287 \mathrm{~nm}$ by a UVspectrophotometer (Perkin-Elmer Corporation, Norwalk, CT). The drug concentration and the percentage of drug release were calculated by the software DissLab ${ }^{\circledR}$.

\subsection{Similarity factor $f_{2}$}

The difference of drug dissolution profiles was evaluated by using $\mathrm{f} 2$ value as described in Equation 5-1.

$$
f_{2}=50 \times \log \left\{\left[1+(1+1 / \mathrm{n}) \sum_{\mathrm{t}=1}\left(\mathrm{R}_{\mathrm{t}}-\mathrm{T}_{\mathrm{t}}\right)^{2}\right]^{-0.5} \times 100\right\} \quad \text { (Eq. 5-1) }
$$

where $f_{2}$ is a similarity factor, $\mathrm{n}$ is the number of sample times, $\mathrm{R}_{\mathrm{t}}$ is the average percentage of drug released at time $t$ for the reference sample, $T_{t}$ is the average percentage of drug released at time $t$ for the test sample. According to FDA's guidance for industry, if $f_{2}$ values were greater than 50 , the two dissolution profiles can be consider as similar or equivalent.

\subsubsection{Mathematic model fitting}

\subsection{Power law equation}

Drug-release mechanism was first studied by fitting the dissolution data to the power law equation [45].

$$
\mathrm{M}_{\mathrm{t}} / \mathrm{M}_{\infty}=\mathrm{k} * \mathrm{t}^{\mathrm{n}}
$$

where $\mathrm{M}_{\mathrm{t}}$ is cumulative amount of drug released at time $\mathrm{t}, \mathrm{M} \infty$ is cumulative amount of drug released at infinite time; $\mathrm{k}$ is release constant incorporating structural and geometric 
characteristics of the system, and $\mathrm{n}$ is the release exponent indicating the mechanism of drug release.

The release exponent ' $n$ ' in the power law takes multiple values based on geometry of the system. For a cylinder shape tablets, if $n<0.45$, the major release mechanism is Fickian diffusion; if the $\mathrm{n}$ value $>0.89$, Case-II transport in which the polymer relaxation is the rate limiting process; if $\mathrm{n}$ value between 0.45 and 0.89 , the "anomalous transport" takes place where both of drug diffusion and swelling of the polymer are involved.

\subsection{Peppas and Shalin equation}

Peppas and Shalin equation is a modification of power law equation [50]. It was performed by decoupling diffusion and "Case-II transport" with the following expression:

$$
\mathrm{M}_{\mathrm{t}} / \mathrm{M}_{\infty}=\mathrm{k}_{1} * \mathrm{t}^{\mathrm{m}}+\mathrm{k}_{2} * \mathrm{t}^{2 \mathrm{~m}}
$$

where $\mathrm{k}_{1}$ and $\mathrm{k}_{2}$ are the release constants and $\mathrm{m}$ is the pure Fickian diffusion exponent. The exponent $\mathrm{m}$ is related to the aspect ratio of the tablets. For the aspect ratio of tablet around 3 , the $m$ value is 0.45 . And $m=0.45$ was used in our study. First term in the right hand of the Equation 5-3 represents the contribution of Fickian diffusion $(\mathrm{F})$ and the second term represents the contribution of tablet structure relaxation (R). R/F ratio can be calculated using Equation 5-4:

$$
\mathrm{R} / \mathrm{F}=\mathrm{k}_{2} * \mathrm{t}^{\mathrm{m}} / \mathrm{k}_{1}
$$

The dominant drug release mechanism can be determined by the R/F ratio at any given time during dissolution. The model fit was performed by the software GraphPad Prism 4.

\subsection{Results and Discussion}

\subsubsection{Propranolol hydrochloride matrix tablets}

According to the dissolution results in Chapter 4, incorporation of the hydrophilic polymer to the matrix significantly increase the drug release at final stage, but the initial burst release still exists. In order to minimize the burst effect for a freely water soluble drug, the roller compaction method was used in this chapter. 


\subsubsection{The effect of roller compaction process on drug release}

The Figure 5-1 displayed the effect of roller compaction of drug with hydrophilic polymers on drug release. Since the dissolution profiles of the direct compression formulations containing 5\% Polyox ${ }^{\circledR} 303$, HPC HXF and HPC GXF were very close (data was not shown). We only selected the direct compression formulation $\mathrm{H} 2(5 \%$ Polyox ${ }^{\circledR}$ 303) for comparison. The results shown in Figure 5-1 demonstrated the drug release rate was affected by the roller compaction process as well as the polymer used in this process. Roller compaction of the drug with the hydrophilic polymer can decrease the drug release rate in this matrix system. This impact was more obvious at the final stage than the initial stage. The hydrophobicity and the viscosity of the hydrophilic polymer also play a role in drug release rate. The tablets prepared by more hydrophobic polymer HPC and the middle viscosity grades of HPC showed a slower release rate than the more hydrophilic polymer Polyox ${ }^{\circledR} 303$ and the high viscosity grade HPC. Table 5-6 listed the initial drug release (1 hour) and the final drug release (12 hours) for these four formulations. The results showed not only a decrease in drug release in initial release for all roller compacted formulations, but also a significant decrease at the final stage. The decrease of the initial release might be due to the freely water soluble drug embedded in the hydrophilic polymer. The viscous gel directly surrounding the drug particles can help to reduce the rapid initial drug release. The hydrophilic polymer with more hydrophobicity and less viscosity has less water absorption initially, therefore less drug diffusion through the gel and an improved initial retarding effect was observed. The decrease of the final drug release might due to reduction in the swellability of the hydrophilic polymer during the roller compaction. Loss of the swellability of the hydrophilic polymer will result in a less porous structure of the final tablets. Therefore incomplete drug release is seen in the dissolution time studied.

\subsubsection{The effect of the intragranular polymer on drug release}

When a controlled release formulation is developed, typically less burst effect and complete release are required. Roller compaction of the drug with the hydrophilic polymer not only limited the burst release, but also suppressed the final release. In order to reach complete release, five percent of Polyox ${ }^{\circledR} 303$ was added extra granularly. The purpose of the extragranular Polyox ${ }^{\circledR} 303$ is to expand the tablet and generate the highly porous tablet structure. Figure 5-2 displayed the effect of extragranular hydrophilic polymer on the drug release. The percentages of initial and final drug release for these four formulations were tabulated in Table 5-7. The extragranular Polyox ${ }^{\circledR} 303$ only increased the final drug release for roller compacted formulations and did not interfere with the initial release. The formulation PR5 prepared with 5\% intragranular low viscosity grade HPC EXF and 5\% extragranular Polyox ${ }^{\circledR} 303$ showed slowest initial release and complete final release. As shown in Table 5-8, the release profile of PR5 meets the USP requirements for propranolol hydrochloride sustained release formulation. 


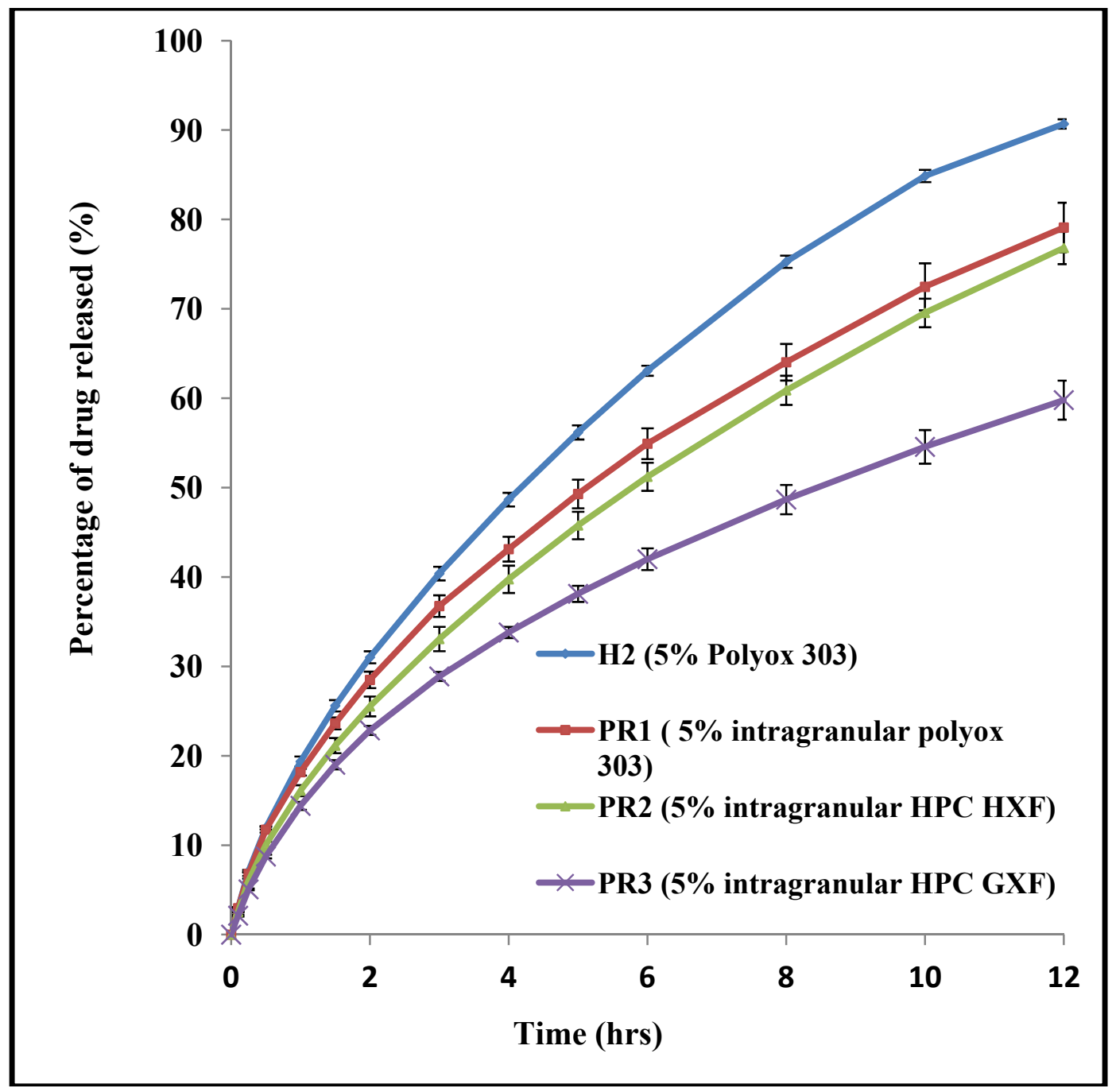

Figure 5-1. Effect of roller compaction on propranolol hydrochloride release 
Table 5-6. The drug release results of formulations containing intragranular hydrophilic polymers at 1 hour and 12 hours

\begin{tabular}{lcc}
\hline Formulations & 1 hour & 12 hours \\
\hline H2 (5\% Polyox ${ }^{\circledR}$ 303) & $19.36 \pm 0.56$ & $90.69 \pm 0.52$ \\
PR1 (5\% intragranular Polyox ${ }^{\circledR}$ 303) & $18.18 \pm 0.39$ & $79.09 \pm 2.77^{*}$ \\
PR2 (5\% intragranular HPC HXF) & $16.10 \pm 0.61^{*}$ & $76.81 \pm 1.81^{*}$ \\
PR3 (5\% intragranular HPC GXF) & $14.40 \pm 0.45^{*}$ & $59.80 \pm 2.18^{*}$ \\
\hline
\end{tabular}

* Statistically significant difference with $\mathrm{H} 2$ formulation. $(\mathrm{P}<0.05)$ 


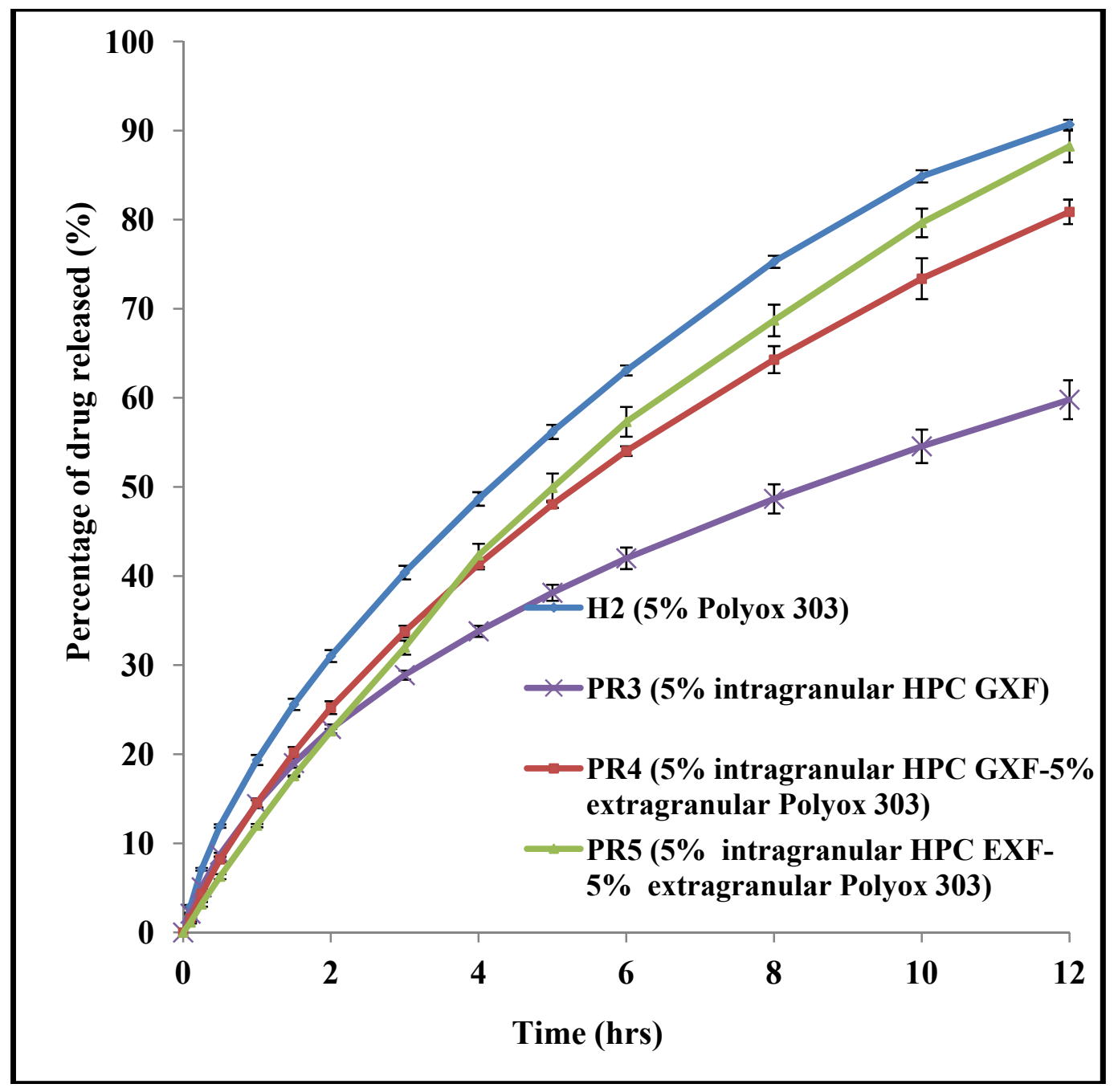

Figure 5-2. Effect of extragranular hydrophilic polymer on propranolol hydrochloride release 
Table 5-7. The drug release results of formulations containing intragranular and extragranular hydrophilic polymers at 1 hour and 12 hours

\begin{tabular}{lcc}
\hline Formulations & 1 hour & 12 hours \\
\hline H2 (5\% Polyox 303) & $19.36 \pm 0.56$ & $90.69 \pm 0.52$ \\
PR3 (5\% intragranular HPC GXF) & $14.40 \pm 0.45^{*}$ & $59.80 \pm 2.18^{*}$ \\
PR4 (5\% intragranular HPC GXF+5\% & $14.54 \pm 0.19^{*}$ & $82.88 \pm 1.78^{*}$ \\
$\begin{array}{l}\text { extra granular Polyox® 303) } \\
\text { PR5 (5\% intragranular HPC EXF+5\% }\end{array}$ & $11.20 \pm 0.51^{*}$ & $88.23 \pm 1.38$ \\
\hline
\end{tabular}

* Statistically significant difference with $\mathrm{H} 2$ formulation. $(\mathrm{P}<0.05)$

Table 5-8. Comparison of propranolol hydrochloride tablets prepared from PR1 and USP specifications

\begin{tabular}{lcc}
\hline Time & Requirements & PR5 \\
\hline $1 \mathrm{~h}$ & $<20 \%$ & $11.20 \pm 0.51$ \\
$3 \mathrm{~h}$ & $20-45 \%$ & $31.97 \pm 0.66$ \\
6h & $45 \%-60 \%$ & $49.90 \pm 0.39$ \\
$12 \mathrm{~h}$ & $>80 \%$ & $88.23 \pm 1.38$ \\
\hline
\end{tabular}




\subsubsection{Mathematic model fit}

The dissolution profiles of formulation H2 (5\% Polyox 303), PR1 (5\% intragranular Polyox $\left.{ }^{\circledR} 303\right)$ and PR5 (5\% intragranular HPC EXF $+5 \%$ extra granular Polyox 303) were fitted to logarithm transformed power law equation. The results were displayed in Figure 5-3. Three dissolution profiles all exhibit good fit for power law equation $\left(\mathrm{R}^{2}>0.99\right)$. The $\mathrm{n}$ values for formulation $\mathrm{H} 2$ and $\mathrm{PR} 1$ falls in the range of 0.45 0.89 which indicates both Fickian diffusion and matrix relaxation are responsible for controlling the drug release. Loss of swellability of hydrophilic polymer in roller compaction results less polymer relaxation, therefore smallest $\mathrm{n}$ value for formulation PR1 was observed. The $\mathrm{n}$ value for formulation PR5 is above 0.89 which indicates the dominant drug release mechanism is matrix relaxation. This result was confirmed by Peppas and Shalin equation (Figure 5-4). The k1 value for formulation PR5 is negative which means the Fickian diffusion can be neglected. This results suggests roller compaction of the freely water soluble drug with the low viscosity hydrophilic polymer can suppress the initial rapid drug diffusion and change the drug release mechanism from Fickian diffusion to matrix relaxation.

\subsubsection{Theophylline anhydrous tablets}

\subsubsection{Formulation development}

Theophylline is a week acid with pka 8.8 [85]. The solubility of theophylline remains constant when the $\mathrm{pH}$ ranges from 1 to 6.8 . The solubility increases as the environmental $\mathrm{pH}$ climbing to 7.0 and above. This is BCS I drug with high solubility and high permeability. According to USP test method, $\mathrm{pH} 7.4$ phosphate buffer is used as the dissolution medium for theophylline sustained release tablets. The formulation development of theophylline sustained release tablets in our study was carried by incorporating different concentration of hydrophilic polymer to the plastic inert matrix system. Three formulations were tried and the dissolution profiles were displayed in Figure 5-5. The formulation $\mathrm{T} 1$ prepared with only plasticized co-processed excipient showed a significant burst release and the incomplete final release. When we incorporated hydrophilic polymer Polyox 303 to the above system, unlike rapid gel diffusion rate of freely water soluble drug propranolol hydrochloride initially, the sparingly water soluble drug theophylline has a limited diffusion through the viscous gel. Therefore the initial release was suppressed by incorporating the hydrophilic polymer. When the concentration of the hydrophilic polymer increased from 5 to $10 \%$, the initial release was further decreased. The significant increase of the final release $(p<0.05)$ were observed for both 5\% and 10\% Polyox 303 formulations. The solubility of theophylline is only one tenth of propranolol hydrochloride and could not generate enough osmotic pressure inside the tablet. A larger amount of hydrophilic polymer was required in order to have a complete final release. The dissolution profile of formulation T3 showed a good satisfaction with the USP requirements as shown in Table 5-9. 


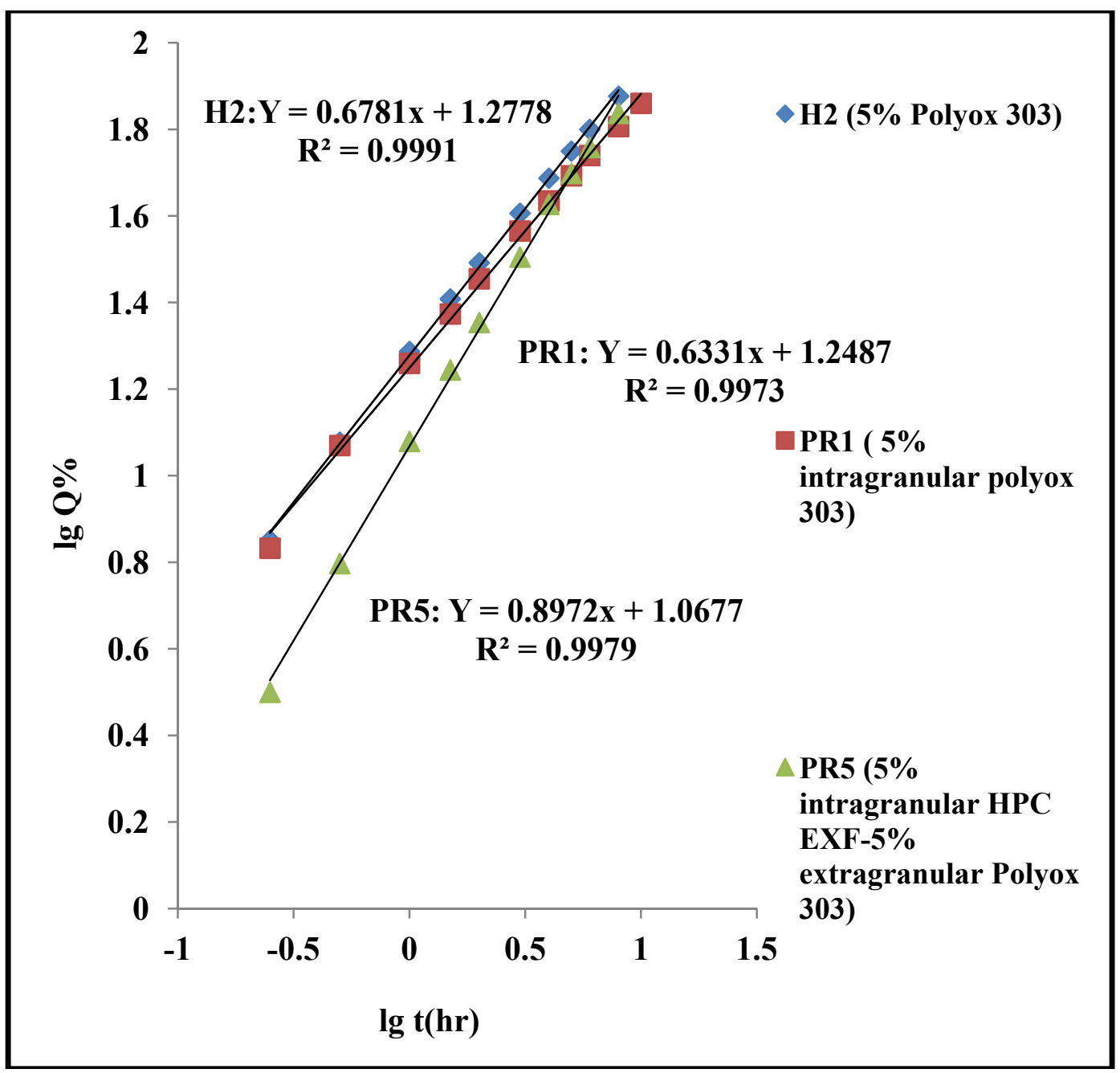

Figure 5-3. Power law equation fit of propranolol hydrochloride release profile 


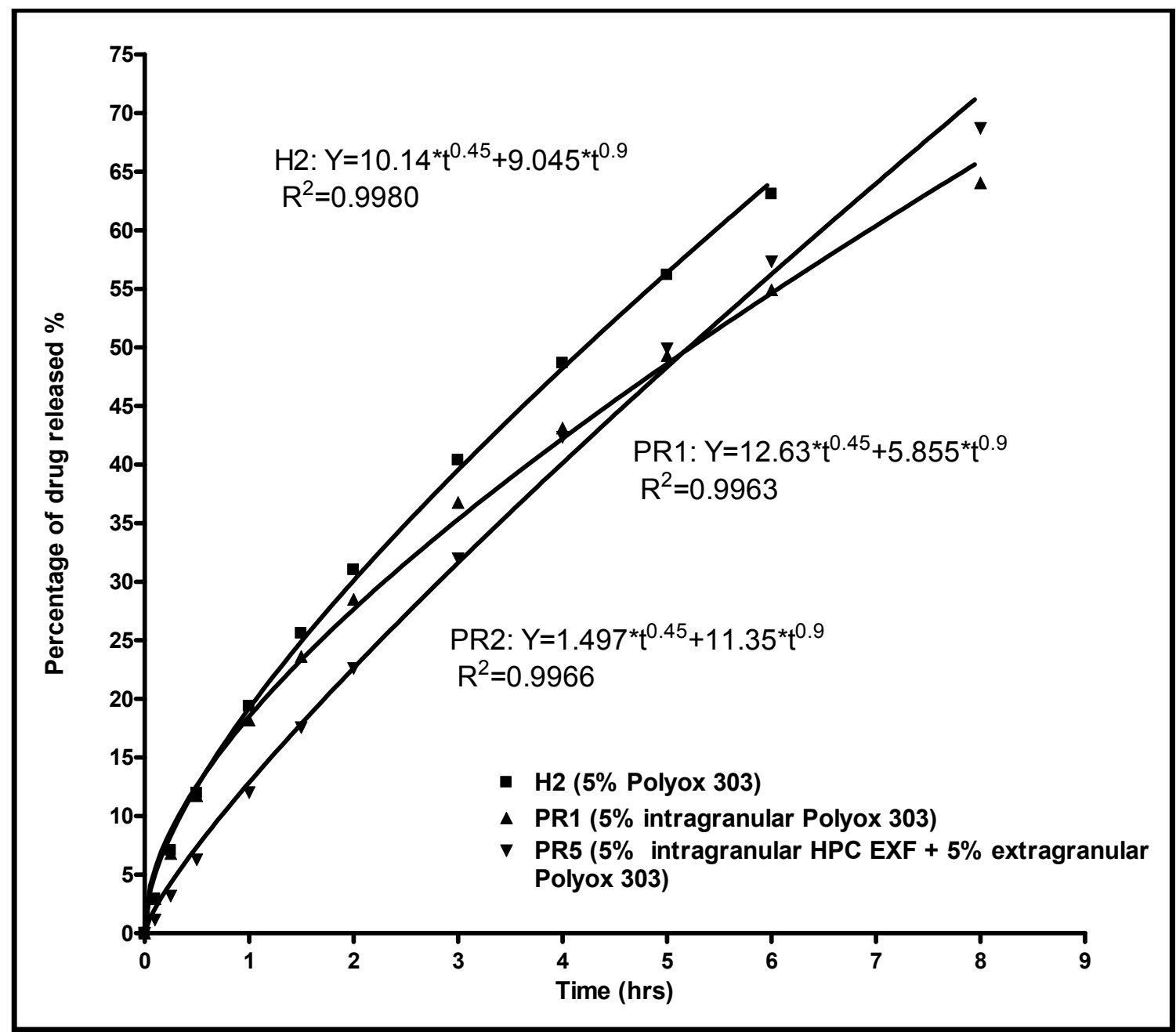

Figure 5-4. Peppas and Shalin equation fit of propranolol hydrochloride release profile 


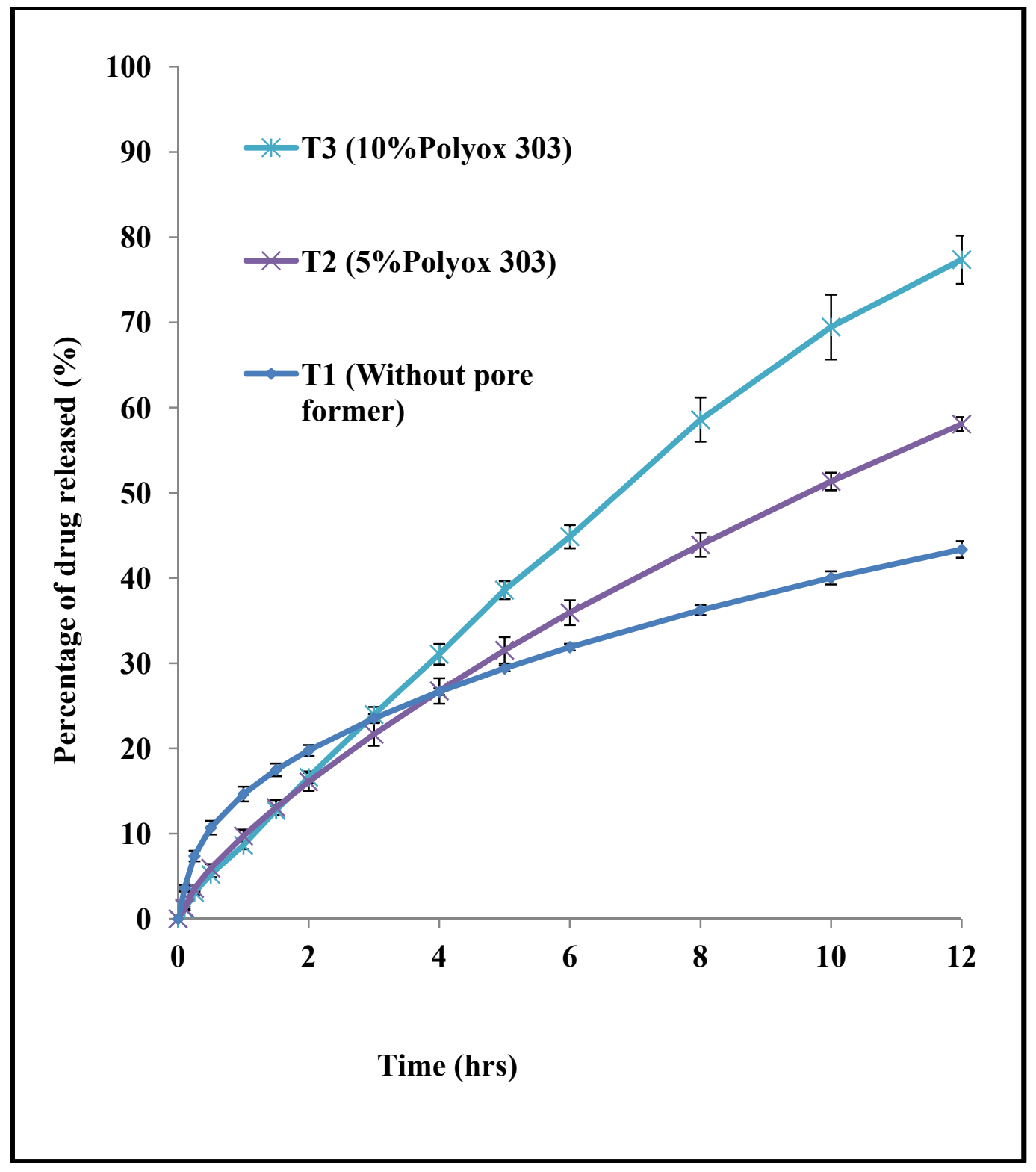

Figure 5-5. Effect of hydrophilic polymer concentrations on theophylline release 
Table 5-9. Comparison of theophylline tablets prepared from $\mathrm{T} 3$ and USP specifications

\begin{tabular}{lcc}
\hline Time & USP requirements & T3 \\
\hline $1 \mathrm{~h}$ & $3-15 \%$ & $8.64 \pm 0.94$ \\
$2 \mathrm{~h}$ & $12-30 \%$ & $16.63 \pm 1.22$ \\
$4 \mathrm{~h}$ & $25-50 \%$ & $31.06 \pm 1.49$ \\
$5 \mathrm{~h}$ & $30-60 \%$ & $38.59 \pm 1.59$ \\
$8 \mathrm{~h}$ & $55-75 \%$ & $58.59 \pm 1.77$ \\
\hline
\end{tabular}




\subsubsection{Mathematic model fit}

The dissolution profiles of three theophylline formulations were fitted to logarithm transformed power law equation as shown in Figure 5-6. Formulation T1 (without hydrophilic polymer) showed a typical diffusion controlled drug release with $\mathrm{n}$ value less than 0.45 . Formulation T2 (5\% Polyox 303) and T3 (10 Polyox 303) all showed the diffusion and structure relaxation combined release mechanism with $\mathrm{n}$ value between 0.45-0.89. The dominant drug release mechanism of T2 and T3 can be determined by the results of Peppas and Shalin equation fit (Figure 5-7). The k2/k1 ratio for T2 is 1.1 which indicates the diffusion is the major mechanism initially and the polymer relaxation is dominant afterwards. The $\mathrm{k} 1$ value for $\mathrm{T} 3$ formulation is negative which indicate the diffusion mechanism is minimal in controlling the drug release. The drug release difference between T2 and T3 can be explained by the difference of gel strength initially. The higher gel strength was obtained by the formulation $\mathrm{T} 3$ which containing more hydrophilic polymer. The higher gel strength effectively prevent the initial burst release for a sparingly water soluble drug theophylline.

\subsubsection{Glipizide tablets}

Glipizide is a weak acid with pka 5.9. It is insoluble in the pure water and shows a $\mathrm{pH}$ dependent solubility. The solubility of glipizide is $0.078 \mathrm{mg} / \mathrm{ml}$ in $\mathrm{pH} 6.8$ buffer and practical insoluble in acidic $\mathrm{pH}$. As suggest in USP, the dissolution test of glipizide sustained release formulations was performed in $0.1 \mathrm{~N}$ hydrochloric acid solution for 2 hours, then $\mathrm{pH} 6.8$ buffer for another 12 hours in our study. The commercialized glipizide sustained release formulation is an osmotic pump tablet named Gluctrol XL ${ }^{\circledR}$. It is bilayer tablet and coated by a semi-permeable membrane with a laser drilled hole on the surface [23]. The drug release can be precisely controlled from this device, but the manufactures of osmotic pump devices is complicated and expensive involving many process variables. In this chapter, we are trying to develop a direct compressed matrix tablet to control the release of glipizide by using the plasticized co-processed excipient.

\subsubsection{The effect of co-processed excipient on drug release}

For development of sustained release inert matrix tablet for a water insoluble drug, a pore former is essential to achieve the complete release. The traditional pore former lactose monohydrate was tried first. In order to obtain complete release for glipizide, a large amount of lactose in the formulation was required and the amount of coprocessed excipient is relatively small. For the purpose of convenience, we used the concentration of co-processed excipient to represent each of the formulation. The dissolution profiles of the reference tablet Gluctrol XL® and tablets prepared with different concentrations of co-processed excipients were displayed in Figure 5-8. Because glipizide is insoluble in acidic $\mathrm{pH}$, all the tablets in Figure 5-8 showed a delayed release profile of two hours. Gluctrol XL® exhibits nearly zero order release between 214 hours. Except the formulation G4 which contains 20\% co-processed excipient, all 


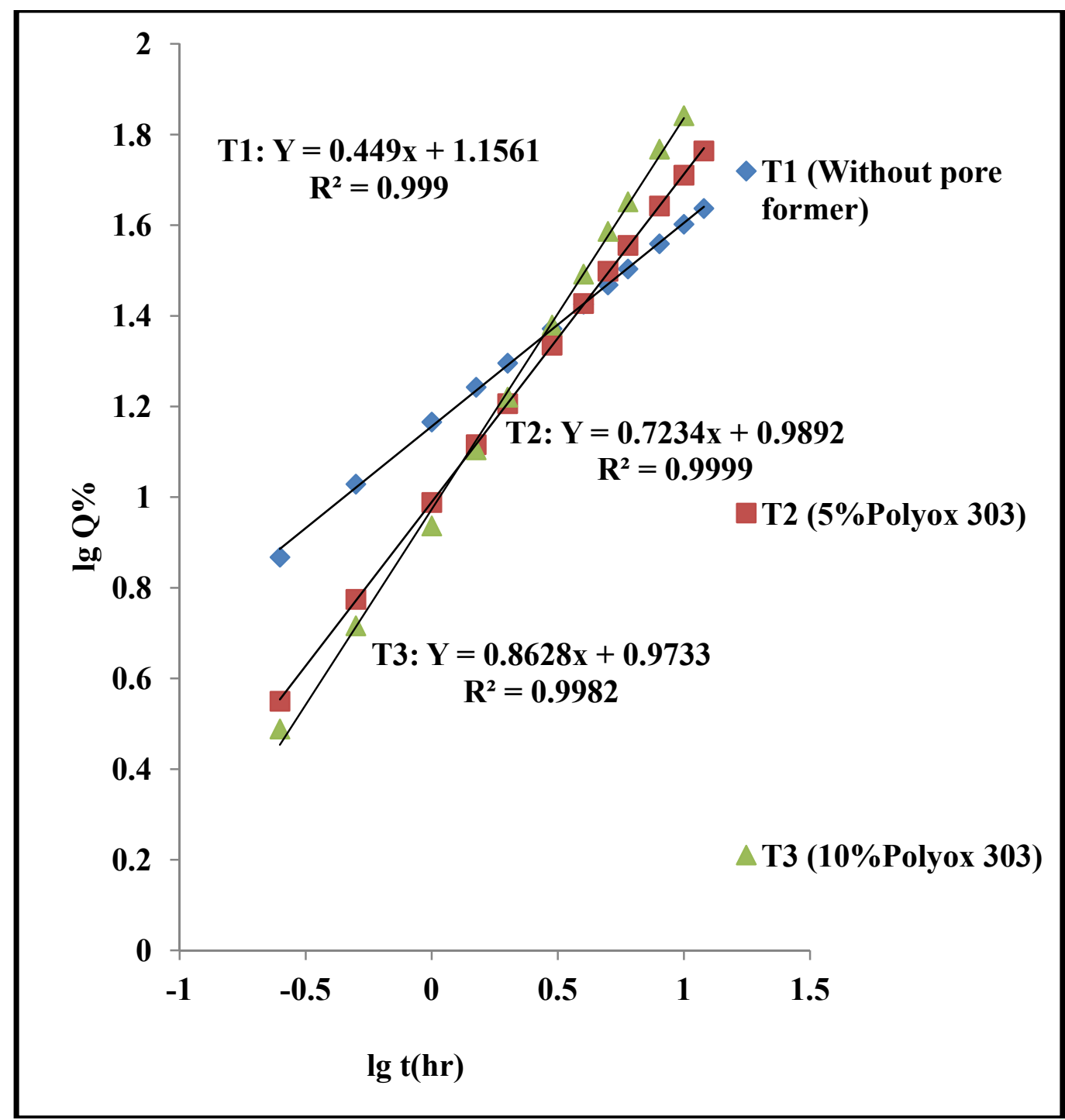

Figure 5-6. Power law equation fit of theophylline release profile 


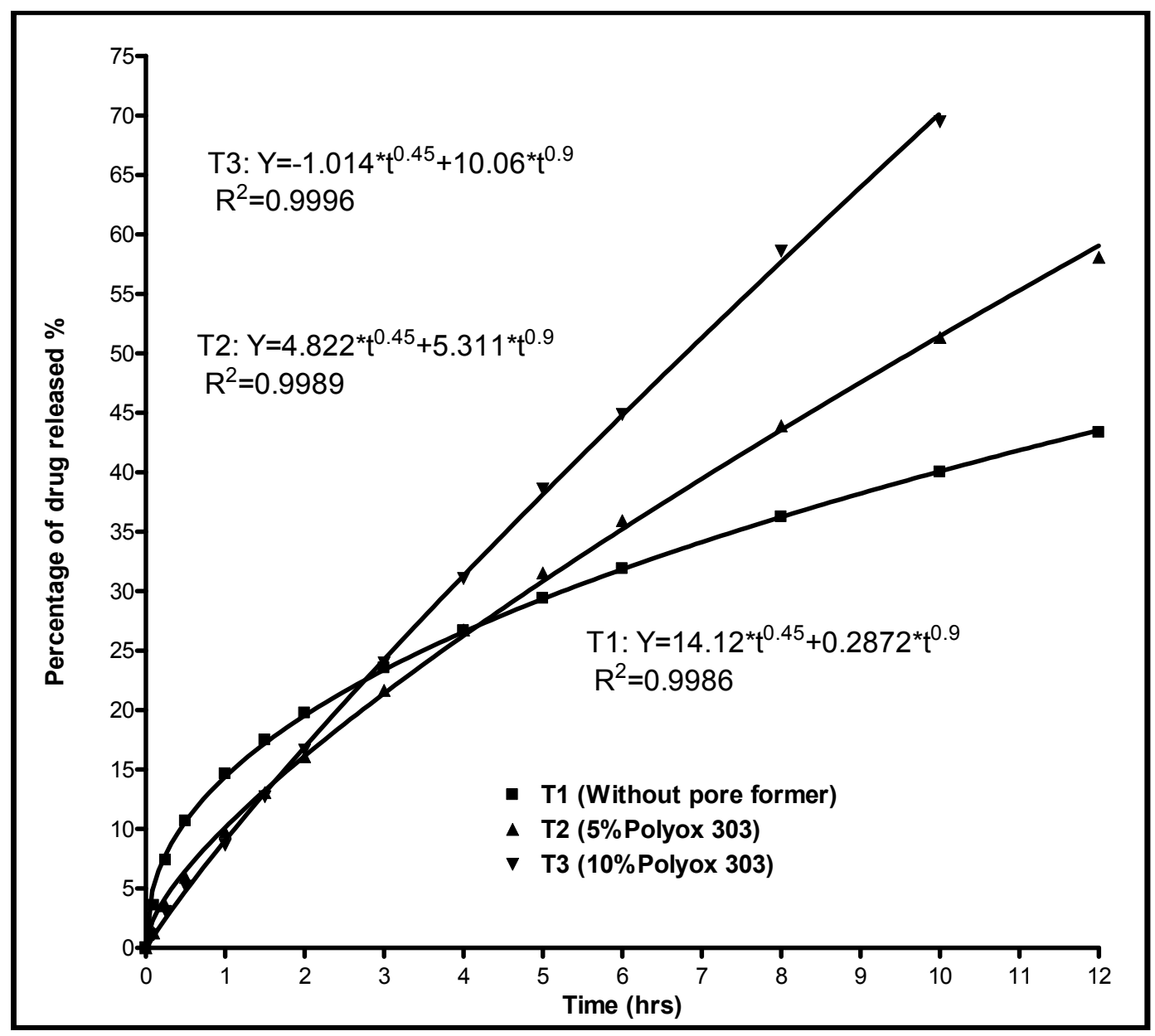

Figure 5-7. Peppas and Shalin equation fit of theophylline release profile 


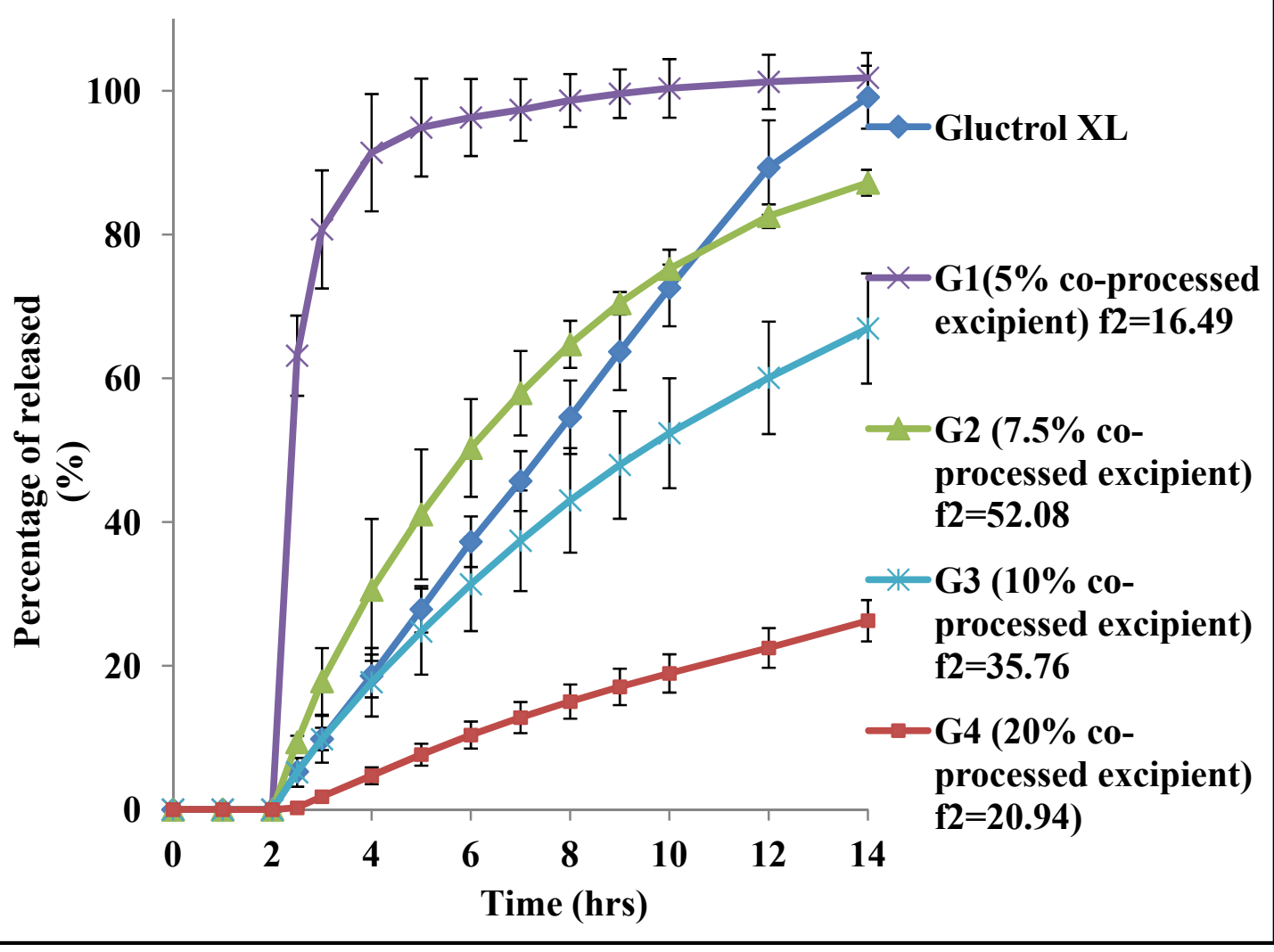

Figure 5-8. Effect of concentrations of co-processed excipient on glipizide release 
other formulations prepared with the co-processed excipient and lactose in Figure 5-8 were disintegrated during dissolution. Because the hydrophobic nature of the glipizide and certain shape and network structure of each disintegrated subunit were maintained during the dissolution, the sustained drug release profile still can be achieved for these formulations. Disintegration of the tablet significantly increases the surface area exposed in dissolution medium and reduces the diffusion pathway of the drug, therefore helping to reach complete final release. But the particle size of each disintegrated subunit is not identical for different tablets prepared with same formulation which causes a large variation in the dissolution within the batches as shown in Figure 5-8. Considering the $f_{2}$ values for each formulation, only formulation $\mathrm{G} 3$ prepared with $7.5 \%$ co-processed excipient is greater than 50. $\left(f_{2}=52.08\right)$ This indicates the dissolution profile of G3 is similar to the reference product Gluctrol XL® and can be further evaluated by an in vivo bioequivalence study.

\subsubsection{The effect of hydrophilic polymer on drug release}

The formulation development of glipizide sustained release matrix tablet was also performed by incorporating different concentrations of the hydrophilic polymer in the matrix system. Since the glipizide is a poorly soluble drug and requires more channels for diffusion, the proper amount of lactose combined with $20 \%$ co-processed excipient were used to construct the matrix system. In order to mimic the 2 hours delayed release as the reference tablet, a $\mathrm{pH}$ sensitive polymer sodium alginate was selected. Sodium alginate is insoluble and cannot swell in acidic $\mathrm{pH}$ but it will hydrate and swell in neutral and basic $\mathrm{pH}$. Incorporating sodium alginate in the matrix tablet can help to keep tablet structure in acid $\mathrm{pH}$ and generate enough porous for drug diffusion in neutral and basic $\mathrm{pH}$. The effect of the concentrations of hydrophilic polymer on drug release was shown in Figure 5-9. All the tablets in Figure 5-9 remained intact during 14 hours dissolution. The drug release rate increasing as the concentration of sodium alginate increased. Since the tablets did not disintegrate during dissolution, less batch variation ( $\mathrm{SD}<4 \%)$ of dissolution was observed. The $f 2$ value for formulation G6 containing $2 \%$ sodium alginate and $20 \%$ co-processed excipient is greater than $50\left(f_{2}=65.03\right)$. This suggests formulation G6 has the similar dissolution profile with reference tablet Gluctrol XL ${ }^{\circ}$.

\subsubsection{Mathematic model fit}

The logarithm transformed power law equation was used to fit the dissolution profiles of formulation G2 and G5 (Figure 5-10). Since all the formulations exhibit two hours delay, the mathematic model fit all took 2 hours as starting point. The formulation G2 (7.5\% co-processed excipient) showed a combined diffusion and structural relaxation controlled drug release mechanism with $\mathrm{n}$ value between 0.45 and 0.89 . The dominant drug release mechanism of G2 can be derived from Peppas and Shalin equation fit (Figure 5-11). The $\mathrm{k} 2 / \mathrm{k} 1$ ratio for $\mathrm{G} 2$ is 1.03 and the $\mathrm{R} / \mathrm{F}$ ratio can be calculated from Equation 5-4. The result indicates the diffusion is the dominant mechanism within the first hour and the structure relaxation is dominant afterwards. Because the disintegrating 


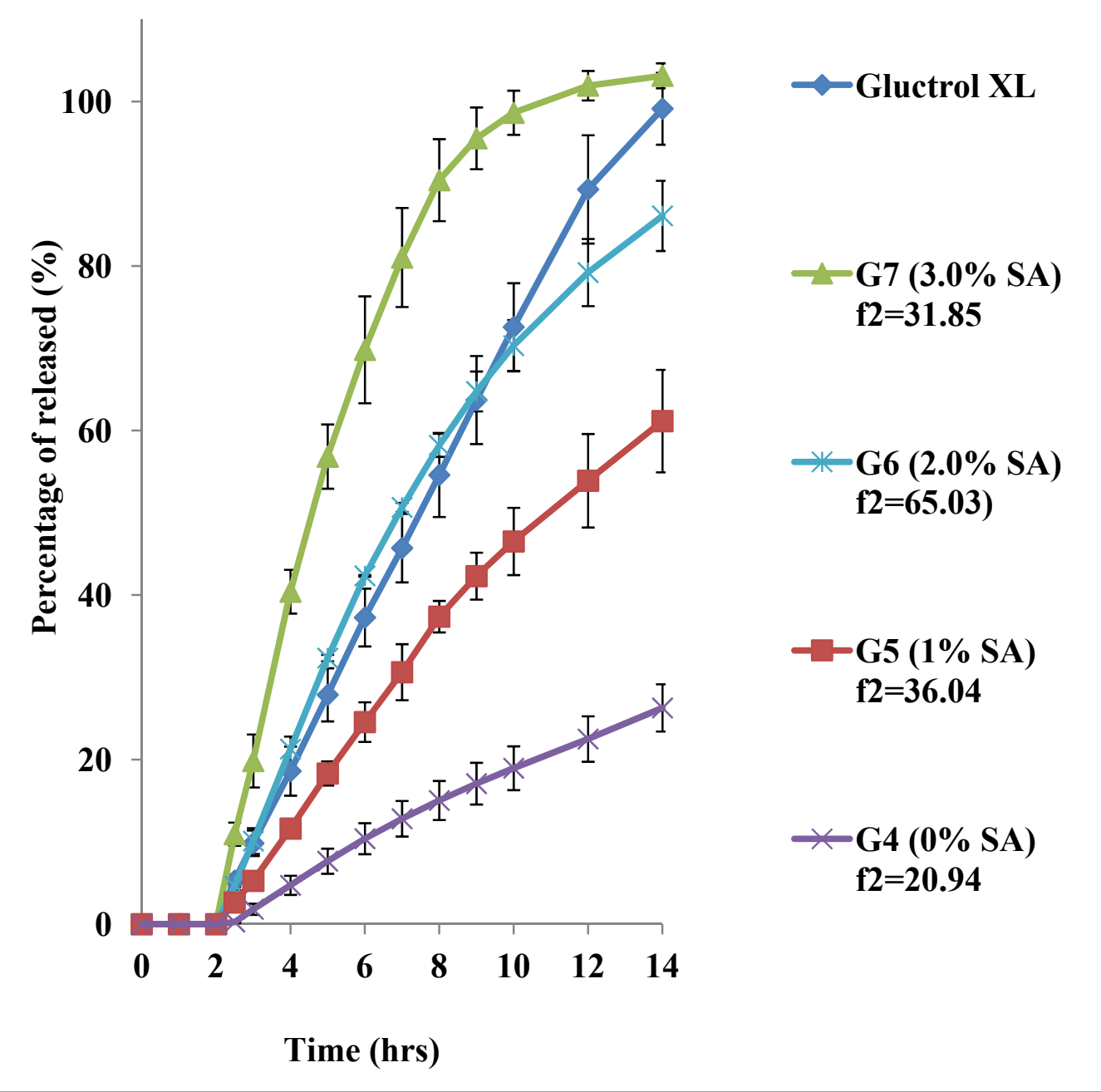

Figure 5-9. Effect of hydrophilic polymer concentrations on glipizide release 


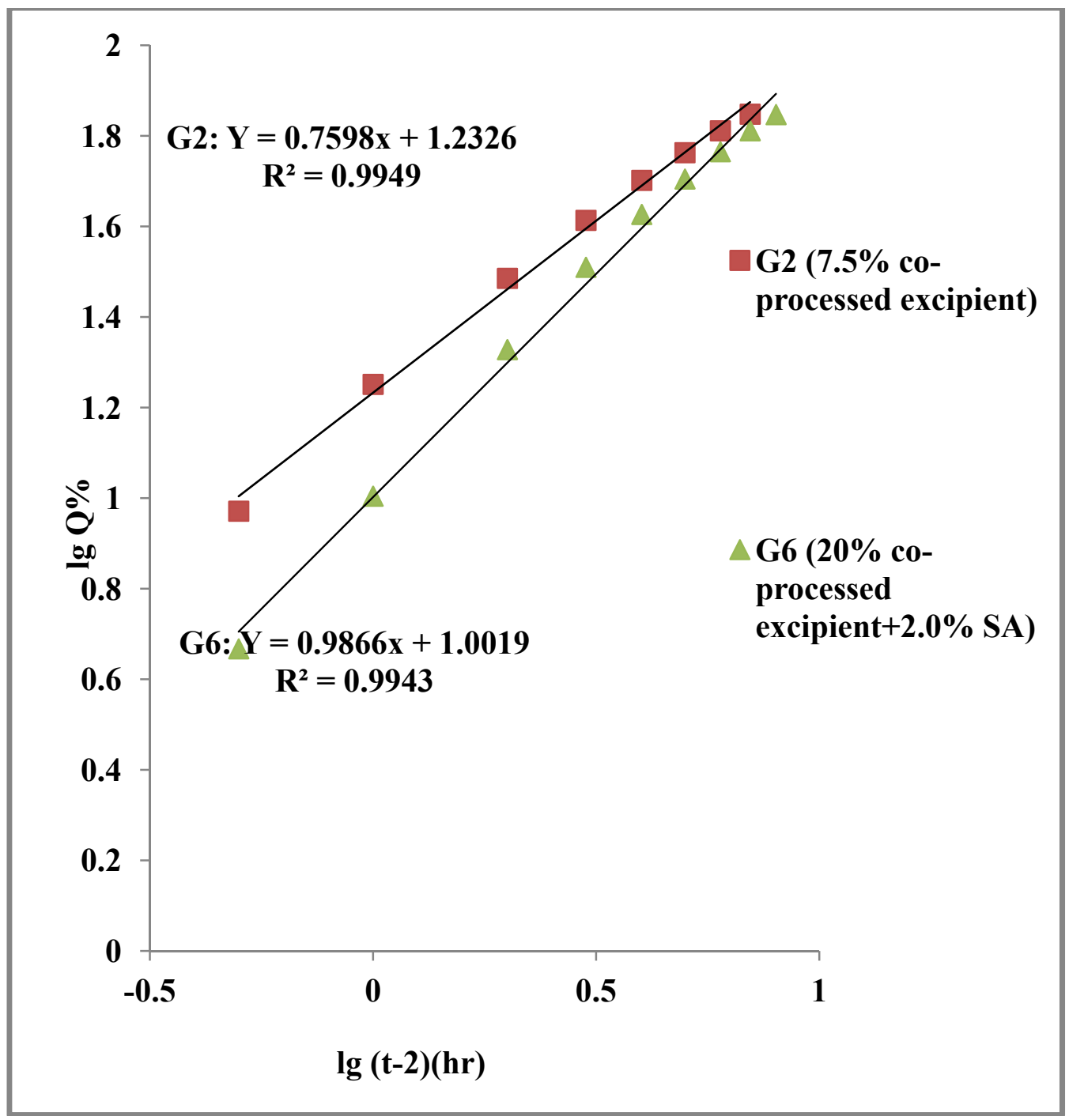

Figure 5-10. Power law equation fit of glipizide release profiles 


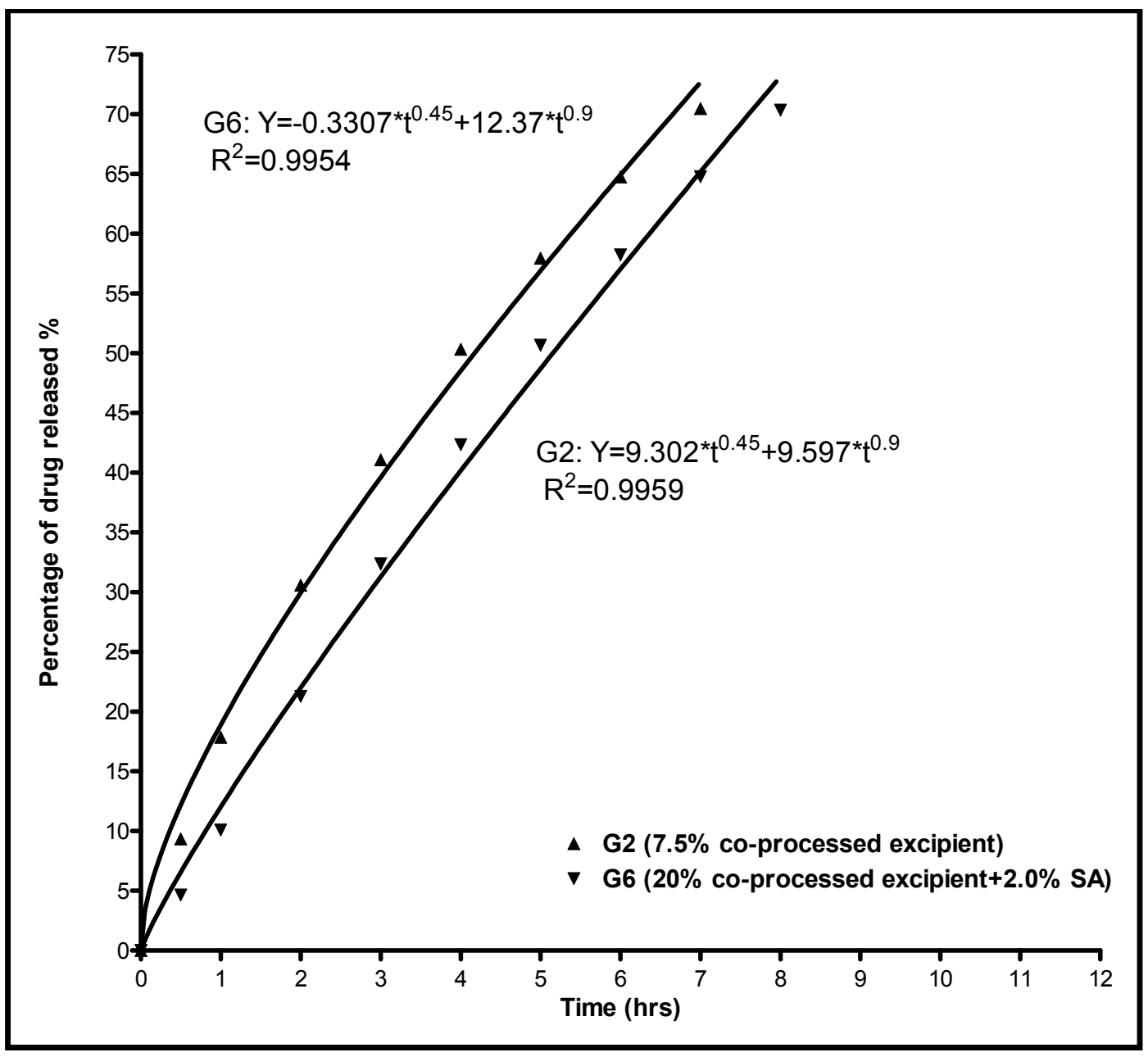

Figure 5-11. Peppas and Shalin equation fit of glipizide release profiles 
of G2 tablets took place in the first hour, the surface drug diffusion is the major release mechanism. After the tablet completely disintegrated, the disintegrated particles were in charge of controlling the drug release and the release mechanism was switched to structure relaxation after 1 hour. Formulation G6 (20\% co-processed excipient $+2.0 \%$ sodium alginate) showed structural relaxation controller drug release with $\mathrm{n}$ value greater than 0.89 . The $\mathrm{k} 1$ value of formulation G6 in Peppas and Shalin equation is negative which confirmed the dominant drug release mechanism is structural relaxation.

\subsection{Conclusions}

The sustained release matrix tablets for three different drugs with different solubility were successfully developed by using the novel swollen porous matrix system. The optimized formulations for these three drugs either meet the USP specifications or have similar dissolution profile as the commercial product. For a freely water soluble drug propranolol hydrochloride, a novel roller compaction method was successfully applied to reduce the initial burst release. The tablets prepared by the roller compacted granules showed suppressed burst release and complete final release. The power law equation and Peppas and Shalin revealed the drug release was controlled by the structure relaxation. For sparingly water soluble drug theophylline, incorporating the hydrophilic polymer can successfully reduce the burst release as well as improve the final release. When $10 \%$ percent of hydrophilic polymer presents in the formulation, the initial burst was further reduced and the drug release mechanism is switched from initial diffusion controlled to completely structure relaxation controlled. Two successful sustained release formulations of poorly water soluble drug glipizide were obtained by using the plasticized co-processed excipient. Both of them have similar dissolution profile as the commercial product Gluctrol XL®. But the formulation prepared with lactose as pore former showed a big variation in dissolution profile while another formulation prepared with both lactose and hydrophilic polymer as pore former exhibited less variation in dissolution profile and higher $f_{2}$ value. 


\section{LIST OF REFERENCES}

1. Tiwari, S.B. and A.R. Rajabi-Siahboomi, Extended-release oral drug delivery technologies: monolithic matrix systems. Methods in Molecular Biology, 2008. 437: p. 217-43.

2. Tiwari, S.B. and A.R. Rajabi-Siahboomi, Modulation of drug release from hydrophilic matrices. Pharmaceutical Technology Asia Pacific, 2008. 2(3): p. 2025.

3. Mariageraldrajan, N., Novel carbopol-wax blends for controlled release oral dosage forms. Dissertation. The University of Tennesses Health Science Center, 2007

4. Pharmaceutical Polymers for Oral Solid Dosage Forms. 2012 [cited 2012 August 6th]. Available from: http://www.lubrizol.com/Pharmaceutical/Brochures.html

5. Nokhodchi, A., Effect of moisture on compaction and compression. Pharmaceutical. Technology, 2005: p. 46-62.

6. Gohel, M. and P.D. Jogani, A review of co-processed directly compressible excipients. Journal of Pharmacy and Pharmaceutical Sciences, 2005. 8(1): p. 7693.

7. Tahara, K., K. Yamamoto, and T. Nishihata, Application of model-independent and model analysis for the investigation of effect of drug solubility on its release rate from hydroxypropyl methylcellulose sustained release tablets. International journal of pharmaceutics, 1996. 133(1): p. 17-27.

8. Nellore, R.V., et al., Development of metoprolol tartrate extended-release matrix tablet formulations for regulatory policy consideration. Journal of Controlled Release, 1998. 50(1): p. 247-256.

9. Ford, J.L., et al., Influence of $\mathrm{pH}$ on the dissolution of promethazine hydrochloride from hydroxypropylmethylcellulose controlled release tablets. Journal of pharmacy and pharmacology, 1985. 37(S12): p. 115P-115P.

10. Miinea, L., et al. A New World of Excipients for Oral Solid Dosage Formulation. 2009 [cited 2012 August 6th]; Available from: http://www.pharmpro.com/Articles/2009/03/A-New-World-of-Excipients-forOral-Solid-Dosage-Formulation/.

11. Higuchi, T., Rate of release of medicaments from ointment bases containing drugs in suspension. Journal of Pharmaceutical Sciences, 1961. 50(10): p. 874-875. 
12. Reynolds, T.D., S.A. Mitchell, and K.M. Balwinski, Investigation of the effect of tablet surface area/volume on drug release from hydroxypropylmethylcellulose controlled-release matrix tablets. Drug Development and Industrial Pharmacy, 2002. 28(4): p. 457-466.

13. Parojcic, J., et al., An investigation into the factors influencing drug release from hydrophilic matrix tablets based on novel carbomer polymers. Drug Delivery, 2004. 11(1): p. 59-65.

14. Moodley, K., et al., Oral Drug Delivery Systems Comprising Altered Geometric Configurations for Controlled Drug Delivery. International journal of molecular sciences, 2011. 13(1): p. 18-43.

15. Chattaraj, S.C. and S.K. Das, Effect of formulation variables on dissolution profile of diclofenac sodium from ethyl-and hydroxypropylmethyl cellulose tablets. Drug Development and Industrial Pharmacy, 1996. 22(7): p. 555-559.

16. Nokhodchi, A., et al., Studies on controlled-release formulations of diclofenac sodium. Drug Development and Industrial Pharmacy, 1997. 23(11): p. 1019-1023.

17. Tiwari, S.B., et al., Controlled release formulation of tramadol hydrochloride using hydrophilic and hydrophobic matrix system. AAPS PharmSciTech, 2003. 4(3): p. 18-23.

18. Traconis, N., et al., Influence of admixed polymers on the metronidazole release from hydroxypropyl methylcellulose matrix tablets. Pharmaceutica Acta Helvetiae, 1997. 72(3): p. 131-138.

19. Khan, G.M., Controlled release oral dosage forms: Some recent advances in matrix type drug delivery systems. The Sciences, 2001. 1(5): p. 350-354.

20. Conti, S., et al., Matrices containing NaCMC and HPMC 1. Dissolution performance characterization. International Journal of Pharmaceutics, 2007. 333(1-2): p. 136-42.

21. Conti, S., et al., Matrices containing NaCMC and HPMC 2. Swelling and release mechanism study. Int J Pharm, 2007. 333(1-2): p. 143-51.

22. Abdelraziq Abdelkareem Adam, F., Design And Evaluation Of Prolonged Release Gliclazide Matrix Tablets. 2007, Universiti Sains Malaysia.

23. Jamzad, S. and R. Fassihi, Development of a controlled release low dose class II drug-Glipizide. International journal of pharmaceutics, 2006. 312(1): p. 24-32.

24. Ansari, M., M. Kazemipour, and J. Talebnia, The development and validation of a dissolution method for clomipramine solid dosage forms. Dissolution Technologies, 2004. 11(3): p. 16-24. 
25. The role of polymer hydrophobicity, molecular weight and drug solubility in modified release matrix system, Aqualon pharmaceutical technology report. 2005, Aqualon.

26. Park, S.H., M.K. Chun, and H.K. Choi, Preparation of an extended-release matrix tablet using chitosan/Carbopol interpolymer complex. International journal of pharmaceutics, 2008. 347(1-2): p. 39-44.

27. Zhang, Y.E., R. Tchao, and J.B. Schwartz, Effect of processing methods and heat treatment on the formation of wax matrix tablets for sustained drug release. Pharmaceutical Development Technology, 2001. 6(2): p. 131-144.

28. Yonezawa, Y., S. Ishida, and H. Sunada, Release from or through a wax matrix system. I. Basic release properties of the wax matrix system. Chemical and pharmaceutical bulletin, 2001. 49(11): p. 1448-1451.

29. Azarmi, S., et al., Thermal treating as a tool for sustained release of indomethacin from Eudragit RS and RL matrices. International journal of pharmaceutics, 2002. 246(1): p. 171-177.

30. Ahmed, S., M. Ashraf, and S. Mohsin, Ethyl Cellulose-Based Solid Matrix System for Sustaining Release of Naproxen. Pakistan Journal of Biological Sciences, 2007. 10(4): p. 668-672.

31. Katikaneni, P.R., et al., Ethylcellulose matrix controlled release tablets of a watersoluble drug. International journal of pharmaceutics, 1995. 123(1): p. 119-125.

32. Sanghavi, N., P. Kamath, and D. Amin, Sustained release tablets of theophylline. Drug Development and Industrial Pharmacy, 1990. 16(11): p. 1843-1848.

33. Engineer, S., Z.J. Shao, and N.A. Khagani, Temperature/humidity sensitivity of sustained-release formulations containing Kollidon ${ }^{\circledR}$ SR. Drug Development and Industrial Pharmacy, 2004. 30(10): p. 1089-1094.

34. Pohja, S., et al., Starch acetate as a tablet matrix for sustained drug release. Journal of Controlled Release, 2004. 94(2): p. 293-302.

35. Gurny, R., E. Doelker, and N. Peppas, Modelling of sustained release of watersoluble drugs from porous, hydrophobic polymers. Biomaterials, 1982. 3(1): $\mathrm{p}$. 27-32.

36. Higuchi, T., Mechanism of sustained - action medication. Theoretical analysis of rate of release of solid drugs dispersed in solid matrices. Journal of Pharmaceutical Sciences, 1963. 52(12): p. 1145-1149.

37. Neau, S.H., et al., The effect of the aqueous solubility of xanthine derivatives on the release mechanism from ethylcellulose matrix tablets. International journal of pharmaceutics, 1999. 179(1): p. 97-105. 
38. Guyonnet, T., C. Brossard, and D. Lefort des Ylouses, Prolongation of theophylline derivative release with cellulose acetate based tablets. J Pharm Belg, 1990. 45(2): p. 111-9.

39. Agrawal, A.M., S.H. Neau, and P.L. Bonate, Wet granulation fine particle ethylcellulose tablets: effect of production variables and mathematical modeling of drug release. The AAPS Journal, 2003. 5(2): p. 48-60.

40. Crowley, M.M., et al., Physicochemical properties and mechanism of drug release from ethyl cellulose matrix tablets prepared by direct compression and hot-melt extrusion. International journal of pharmaceutics, 2004. 269(2): p. 509-522.

41. Dabbagh, M.A., et al., Effects of polymer particle size, compaction pressure and hydrophilic polymers on drug release from matrices containing ethylcellulose. International journal of pharmaceutics, 1996. 140(1): p. 85-95.

42. Sadeghi, F., F. Mosaffa, and H.A. Garekani, Effect of Particle Size, Compaction Force and Presence of Aerosil 200 on the Properties of Matrices Prepared from Physical Mixture of Propranolol Hydrochloride and Eudragit RS or RL. Iranian Journal of basic medical science 2007.

43. Qiu, Y. and G. Zhang, Development of Modified-Release Solid Oral Dosage Forms, in Developing Solid Oral Dosage Forms: Pharmaceutical Theory and Practice, 2009. p. 290-305.

44. Patel Kundan K, P.M.S., Bhatt Nayana M, Patel Laxmanbhai D, Pathak and N.L.a.P. Kanu, An Overview Extended Release Matrix Technology. International journal of chemical and pharmaceutical sciences, 2012. 1(2): p. 828-843.

45. Ritger, P.L. and N.A. Peppas, A simple equation for description of solute release I. Fickian and non-Fickian release from non-swellable devices in the form of slabs, spheres, cylinders or discs. Journal of Controlled Release, 1987. 5(1): p. 2336.

46. Ritger, P.L. and N.A. Peppas, A simple equation for description of solute release II. Fickian and anomalous release from swellable devices. Journal of Controlled Release, 1987. 5(1): p. 37-42.

47. Rao, P.R. and P.V. Diwan, Permeability studies of cellulose acetate free films for transdermal use: influence of plasticizers. Pharmaceutica Acta Helvetiae, 1997. 72(1): p. 47-51.

48. Meier, M.M., L.A. Kanis, and V. Soldi, Characterization and drug-permeation profiles of microporous and dense cellulose acetate membranes: influence of plasticizer and pore forming agent. International journal of pharmaceutics, 2004. 278(1): p. 99-110. 
49. El-Arini, S.K. and H. Leuenberger, Dissolution properties of praziquantel-PVP systems. Pharmaceutica Acta Helvetiae, 1998. 73(2): p. 89-94.

50. Peppas, N.A. and J.J. Sahlin, A simple equation for the description of solute release. III. Coupling of diffusion and relaxation. International journal of pharmaceutics, 1989. 57(2): p. 169-172.

51. Costa, P. and J.M. Sousa Lobo, Modeling and comparison of dissolution profiles. Eur J Pharm Sci, 2001. 13(2): p. 123-33.

52. Hopfenberg, H.B., D.R. Paul, and F.W. Harris, Controlled release polymeric formulations, in ACS Symposium 1976, American Chemical Society: Washington, DC,

53. Yuan, J., et al., Formulation Effects on the Thermomechanical Properties and Permeability of Free Films and Coating Films: Characterization of Cellulose Acetate Films. Pharmaceutical technology, 2009. 33(3): p. 88-100.

54. Guo, J.H., An investigation into the formation of plasticizer channels in plasticized polymer films. Drug Development and Industrial Pharmacy, 1994. 20(11): p. 1883-1893.

55. Liu, L., et al., Preparation and characterization of cellulose acetate membrane for monolithic osmotic tablet. Korea Polymer Journal, 1999. 7: p. 289-296.

56. Liu, L., et al., Monolithic osmotic tablet system for nifedipine delivery. Journal of Controlled Release, 2000. 67(2): p. 309-322.

57. Herbig, S., et al., Asymmetric-membrane tablet coatings for osmotic drug delivery. Journal of Controlled Release, 1995. 35(2): p. 127-136.

58. Yuan, J. and S.H.W. Wu, Sustained-release tablets via direct compression: A feasibility study using cellulose acetate and cellulose acetate butyrate. Pharmaceutical technology, 2000. 24(10): p. 92-106.

59. Agabeyoglu, I. and N. Kaynar-Özdemir, Studies on sustained release VII. Inert matrix tablets of zomepirac sodium with cellulose acetate. STP Pharma, 1990. 6: p. 629-634.

60. El-Khawas, M., K. El-Khordairy, and M. Samaha, Phenylpropanolamine controlled-release tablets: in vitro availability. Pharmazeutische Industrie, 1993. 55(4): p. 392-395.

61. Picker, K.M. and F. Bikane, Tablet formation and release from matrix tablets manufactured with cellulose acetate. International journal of pharmaceutics, 1998. 175(2): p. 147-164. 
62. Soppimath, K., A. Kulkarni, and T. Aminabhavi, Encapsulation of antihypertensive drugs in cellulose-based matrix microspheres: characterization and release kinetics of microspheres and tableted microspheres. Journal of Microencapsulation, 2001. 18(3): p. 397-409.

63. Choudhury, P.K., M. Kar, and C.S. Chauhan, Cellulose acetate microspheres as floating depot systems to increase gastric retention of antidiabetic drug: formulation, characterization and in vitro-in vivo evaluation. Drug Development and Industrial Pharmacy, 2008. 34(4): p. 349-354.

64. Soppimath, K.S., A.R. Kulkarni, and T.M. Aminabhavi, Development of hollow microspheres as floating controlled-release systems for cardiovascular drugs: preparation and release characteristics. Drug Development and Industrial Pharmacy, 2001. 27(6): p. 507-515.

65. Hoy, M.R. and E.J. Roche, Taste mask coatings for preparation of chewable pharmaceutical tablets. 1996, US Patent 5489436: USA.

66. Yu, D. and E. Roche, Taste masked pharmaceutical liquid formulations. 2002, US Patent 6482823: USA.

67. Rao, R. and P. Diwan, Drug diffusion from cellulose acetate-polyvinyl pyrrolidone free films for transdermal administration. Indian Journal of Pharmaceutical Science, 1996. 58(6): p. 246.

68. Hardy, I., W. Cook, and C. Melia, Compression and compaction properties of plasticised high molecular weight hydroxypropylmethylcellulose (HPMC) as a hydrophilic matrix carrier. International journal of pharmaceutics, 2006. 311(1): p. 26-32.

69. Xiao, C., Plasticized ethyl cellulose for preparation of controlled release tablets. Dissertation. The University of Tennesses Health Science Center, 2007

70. Yuan, J. Several Factors to consider when selecting a pharmaceutical excipient. 2007 [cited 2012 August 6th]; Available from: www.eastman.com/literature_center/p/pci111.pdf

71. Panda, B., et al. Co-processed excipients: an overview of formulation aspects, physical characteristics and role as a pharmaceutical-aid. 2011 [cited 2012 August 6th]; Available from: http://www.pharmatutor.org/articles/overview-ofco-processed-pharmaceutical-excipients-formulation-aspects.

72. Lawrence H. Block, R.C.M., Shireesh P. Apte, Richard H. Wendt, Eric J. Munson,Joseph R. Creekmore, and C.S. Indira V. Persaud, and Hong Wang, CoProcessed excipients. Pharmacopeial Forum, 2009. 35(4): p. 1026-1028.

73. Nachaegari, S.K. and A.K. Bansal, Coprocessed excipients for solid dosage forms. Pharmaceutical technology, 2004. 28(1): p. 52-65. 
74. Edgar, K.J., et al., Advances in cellulose ester performance and application. Progress in polymer science, 2001. 26(9): p. 1605-1688.

75. BASF, Kollindon® SR. 2011 [cited 2012 Aug 6th]; Available from: http://www.pharma-ingredients.basf.com/Statements/

76. GBI, R. Oral Drug Delivery Market - Controlled and Sustained Release to be Major Revenue Generators. 2010 [cited 2012 August 6th]; Available from: http://www.marketreports.com/Sample/Global_Data/GBIHC050MR_sample.pdf

77. Taylor, M.K., et al., Composite method to quantify powder flow as a screening method in early tablet or capsule formulation development. AAPS PharmSciTech, 2000. 1(3): p. 20-30.

78. Heckel, R., An analysis of powder compaction phenomena. Transactions of the Metallurgical Society of AIME, 1961. 221: p. 1001-1008.

79. Heckel, R., Density pressure relationship in powder compaction. Trans Metallurgical Soc of AIME, 1961. 221: p. 671-675.

80. Sun, C.C., Mechanism of moisture induced variations in true density and compaction properties of microcrystalline cellulose. International journal of pharmaceutics, 2008. 346(1-2): p. 93-101.

81. Peppas, N.A., A model of dissolution - controlled solute release from porous drug delivery polymeric systems. Journal of biomedical materials research, 1983. 17(6): p. 1079-1087.

82. Frenning, G., Modelling drug release from inert matrix systems: from movingboundary to continuous-field descriptions. International Journal of Pharmaceutics, 2011. 418(1): p. 88-99.

83. Ubrich, N., et al., Preparation and characterization of propranolol hydrochloride nanoparticles: a comparative study. Journal of Controlled Release, 2004. 97(2): p. 291-300.

84. Verma, R.K. and S. Garg, Development and evaluation of osmotically controlled oral drug delivery system of glipizide. European journal of pharmaceutics and biopharmaceutics, 2004. 57(3): p. 513-525.

85. Simon, L., et al., Effect of $\mathrm{pH}$ on Theophylline Release from Partially Esterifted Alginic Acid Matrices. Drug Development and Industrial Pharmacy, 1994. 20(15): p. 2341-2351. 


\section{VITA}

Yinqi Zhou was born in 1976 in Hunan province, China. She received her Bachelor degree in Traditional Chinese Medicine from Hunan University of Traditional Chinese Medicine, China in June 1996 and Master degree in Pharmaceutical Sciences from China Pharmaceutical University, China in June 2001. She joined the graduate school at University of Tennessee Health Science Center in the fall of 2007. She is a member of Rho Chi Pharmaceutical Honor Society, The National Scholars Honor Society, American Association of Pharmaceutical Scientists (AAPS) and Controlled Release Society (CRS). 